\title{
Molecular Diffusion From Bone into Cartilage: A Study of the Osteochondral Junction
}

by

Nadine Vautour

A thesis submitted to the Faculty of Graduate and Postdoctoral Affairs in partial fulfillment of the requirements for the degree of

Master of Applied Science

in

Biomedical Engineering

Carleton University

Ottawa, Ontario

(C) 2017, Nadine Vautour 


\begin{abstract}
Osteoarthritis is an idiopathic joint disease that affects $13 \%$ of Canadians. To gain a better understanding of the biochemical relationship between the bone and cartilage, molecular diffusion across the bone-cartilage junction was assessed. Passive diffusion of a small (605 Da), nutrient-sized, contrast agent across the osteonchondral junction was successfully measured using contrast-enhanced MRI. This method could be employed for future in vivo studies. The osteochondral junction was simulated using a finite difference method to assess the subchondral bone as a barrier. The average resistivity value was $1.65 \times 10^{-6} \mathrm{~s}^{-1}$. Inclusion of resistivity in the simulation produced a better fit of the experimental data. The osteochondral junction's permeability to larger, protein-sized, solutes was assessed by attempting to diffuse fluorescent tracers $(3-70 \mathrm{kDa})$. The selected fluorescent tracers were unable to passively diffuse across the osteochondral junction. This thesis provided a better understanding of the crosstalk between bone and cartilage.
\end{abstract}




\section{Acknowledgements}

I would first like to express my gratitude to my supervisor, Andrew for his time, guidance and knowledge along the way. He has been tremendously helpful in guiding this work and overcoming various problems that arose throughout this thesis. I thank Andrew for all his patience, perseverance and expertise in cartilage and biomechanics.

I also thank Alex Proctor and his team in the machine shop for helping me machine the diffusion chambers used throughout this research. I thank Greg Cron for his time in setting up the MR imaging and for his expertise in the magnetic resonance field. I also would like to thank Chloë van Oostende-Triplet, for her expertise in fluorescent imaging and meeting with me on a weekly basis while we tried various approaches to diffusing fluorescent tracers. I thank Dr. Paul Beaulé and his team for their work in obtaining human samples for this research.

Mohammed Abdelazez, thank you for always talking me through my problems and offering solutions. Most importantly, thank you for your willingness to help and for making this degree much more enjoyable. I also thank Kevin Dick for co-chairing the Carleton chapter of the Engineers in Medicine and Biology Society with me and all the fun we had in and out of the lab. I will miss being a part of this lab.

To all my friends, colleagues, professors who supported me throughout this degree, thank you.

This last year was undoubtedly the hardest year for my family. Life is unpredictable and things don't always happen the way we wanted. Taking a semester off was not easy and returning to Ottawa to finish this project seemed impossible at times. Despite this and all the challenges that we still face, my family has continued to support and encourage me 
throughout this degree. I owe my deepest gratitude to my parents, Claude and Nicole. They're unwavering support, pride and interest in my work has truly kept me motivated. To my brother, Stéphane, thank you for always being there for me and constantly reminding me that I am the "smart one" and I could do this. 


\section{Table of Contents}

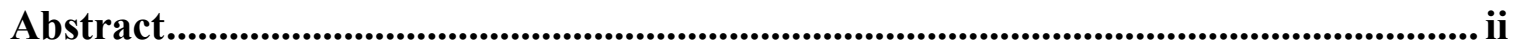

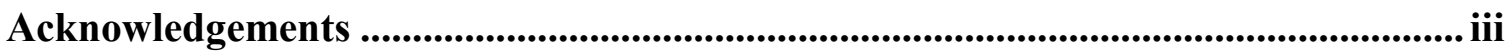

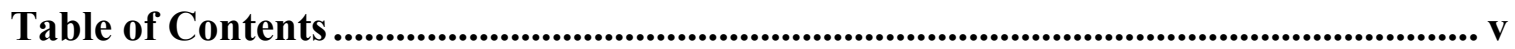

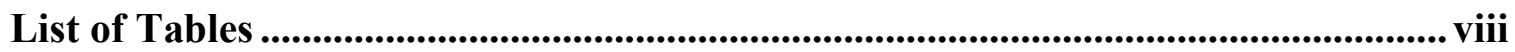

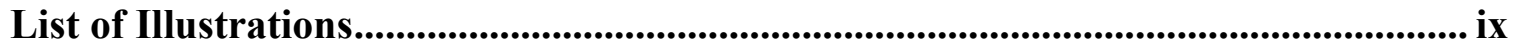

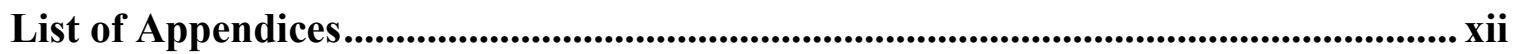

Acronyms ............................................................................................................................................. xiii

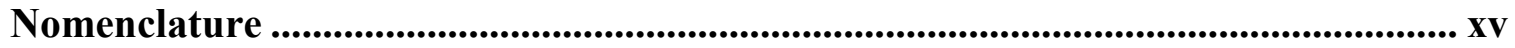

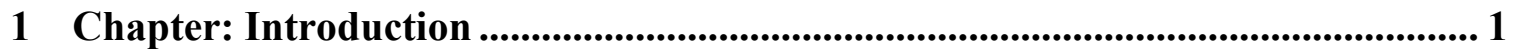

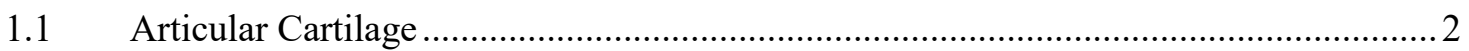

1.1.1 Composition and Structure of Articular Cartilage ................................................2

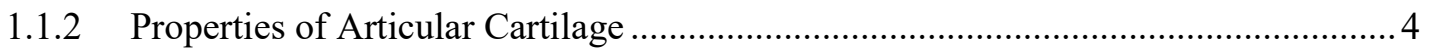

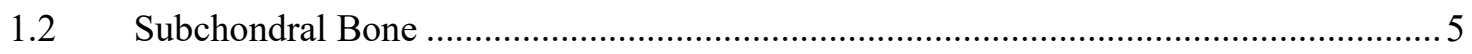

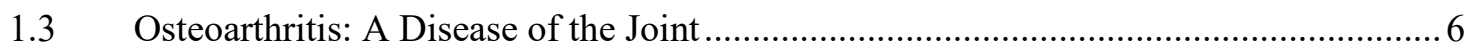

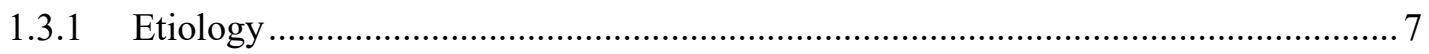

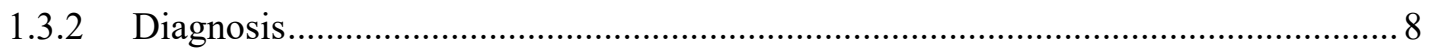

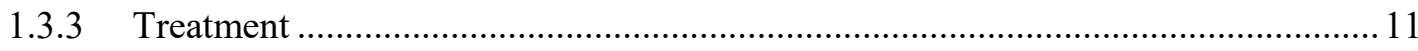

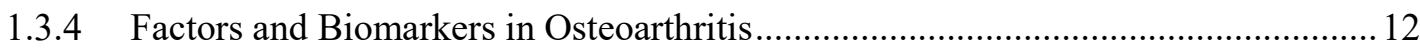

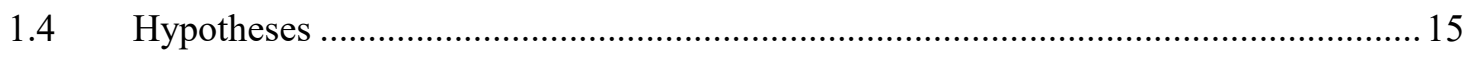

\section{Chapter: Diffusivity Assessment Across the Osteochondral Junction Using}

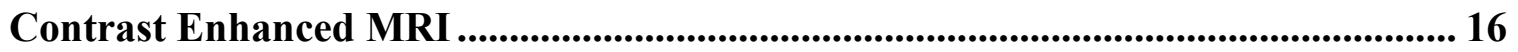

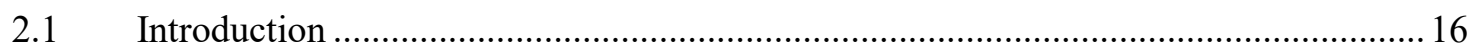

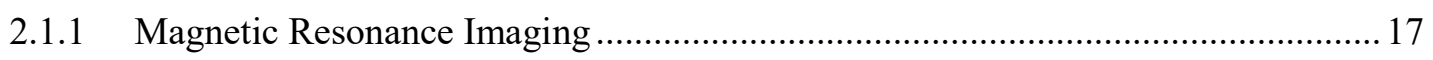

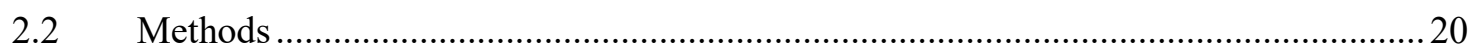




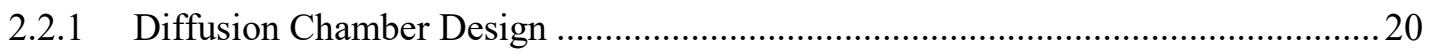

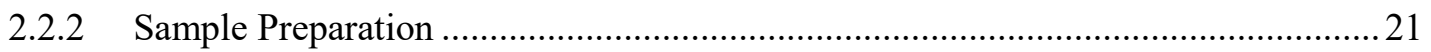

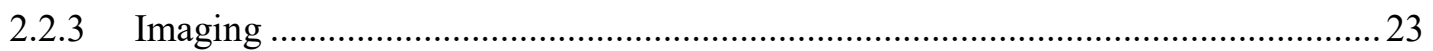

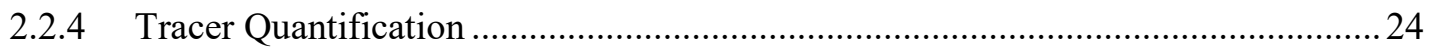

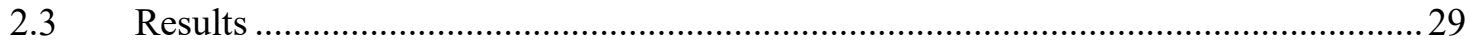

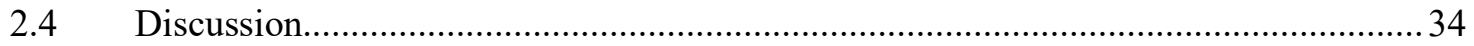

3 Chapter: Numerical simulation of diffusion in osteochondral samples................ 42

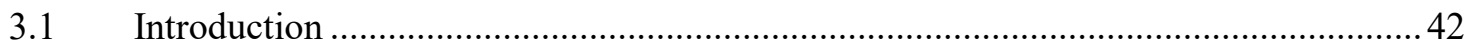

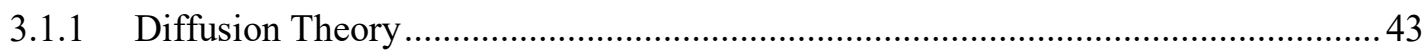

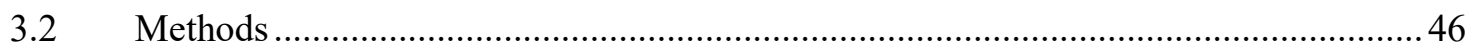

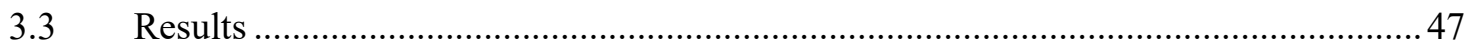

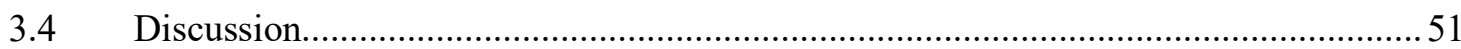

4 Chapter: Fluorescence Spectroscopy ....................................................................... 55

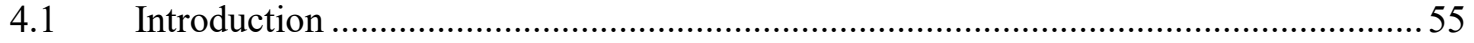

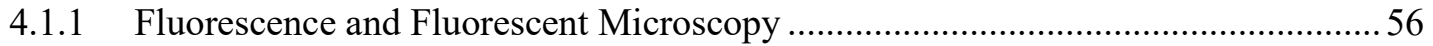

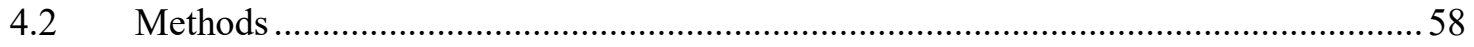

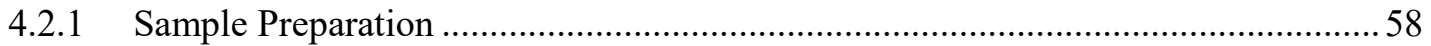

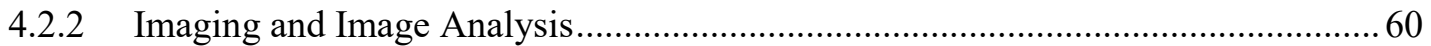

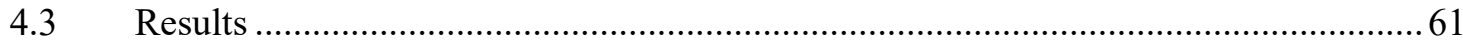

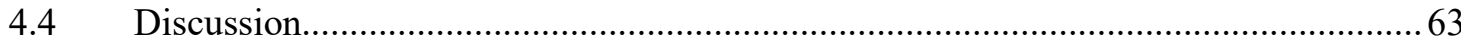

5 Chapter: Discussion and Conclusions ................................................................ 67

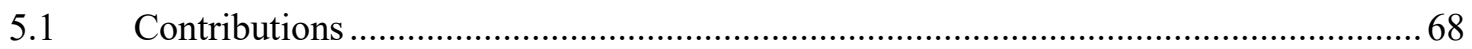

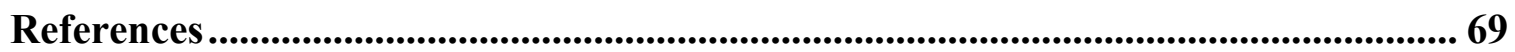

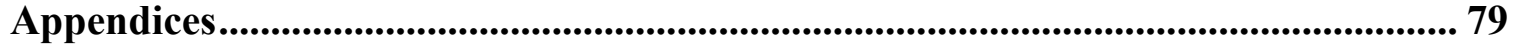

Appendix A - Diffusion chamber engineering drawing .......................................................... 79 
Appendix B - Experimental Gd concentration at select cartilage depths and times for all samples

Appendix C - Simulated Gd concentration fit to experimental results at select cartilage depths and times, for all samples

Appendix D Simulated Gd concentration without the bone barrier fit to experimental results at select cartilage depths and times, for all samples. 88 


\section{List of Tables}

Table 1 Kellgren-Lawrence OA grading scale [8]....................................................... 10

Table 2 Biomarkers and indicative factors of osteoarthrtitis $[13,64-70] \ldots \ldots \ldots \ldots \ldots \ldots \ldots \ldots . . . .14$

Table 3 MRI Imaging times for samples 1-7 ............................................................ 24

Table 4 Number of ROIs for each sample ................................................................. 25

Table 5 Relaxivity values of cartilage and water..................................................... 27

Table 6 Diffusivity values of Samples 1-7 for select times ................................................ 31

Table 7 Diffusivity values of Samples 1-7 by distance from the tidemark ...................... 31

Table 8 Experimental and simulated average diffusivity values at select times.

Experimental values were determined in Chapter 2 ……........................................ 49

Table 9 RMS error and relative error of simulated concentrations with and without

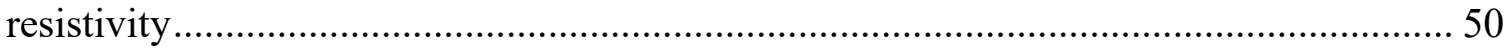

Table 10 Best fit resisitivity values of each sample ......................................................... 50

Table 11 Excitation and emission values for fluorescein and Texas Red [111, 112]...... 59

Table 12 Sample and tracer information for fluorescent tracer diffusion experiments .... 59

Table 13 Summary of findings for Fluorescent experiment (Specimens 1-10)................ 63 


\section{List of Illustrations}

Illustration 1 Articular cartilage zones: superficial, middle and deep .............................. 4 Illustration 2 (A) Hydrogen atoms, with the axis representing the magnetic moment, M,

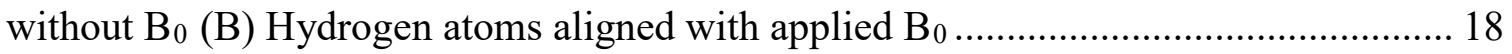
Illustration 3 Diffusion chamber model ................................................................... 20

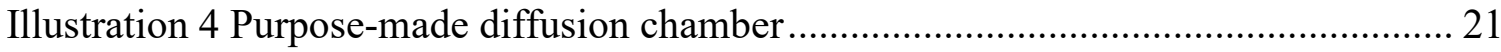

Illustration 5 Osteochondral porcine plug (diameter of $10 \mathrm{~mm}$, thickness of $4 \mathrm{~mm}$ )...... 22 Illustration 6 MR image ROI selection for Sample 2 ……….................................... 25

Illustration 7 Relationship between cartilage MR signal and gadobutrol concentration .. 27 Illustration 8 Relationship between bone MR signal and gadobutrol concentration........ 28

Illustration 9 Sample 2 MR image at 0 hours and 44.25 hours......................................... 29

Illustration 10 Average Gd concentration at select times and cartilage depths for all

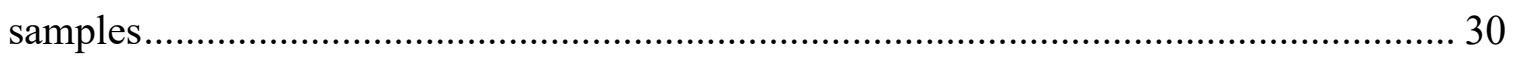

Illustration 11 Diffusivity of Gd through cartilage for durations up to 50 hours ............. 33 Illustration 12 Diffusivity values of Gd through cartilage at select distances from the

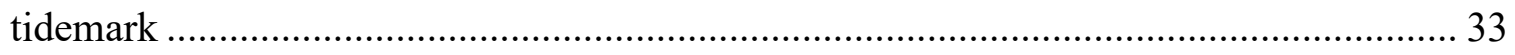

Illustration 13 Comparison of first and last images of sample 2 .................................... 34

Illustration 14 32-bit image of Sample 2 at 0 hours..................................................... 37

Illustration 15 16-bit image of sample 2 at 0 hours ....................................................... 38

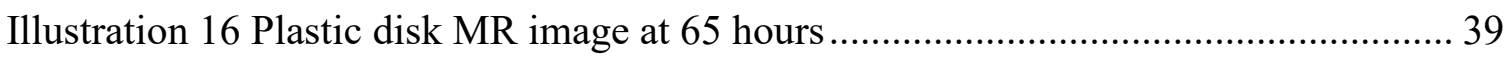

Illustration 17 Plastic disk MR image at 0 hours ........................................................... 40

Illustration 18 Image intensity of control sample ……………...................................... 40

Illustration 19 Model of diffusion setup for FD analysis and simulation ......................... 44 
Illustration 20 Simulated best fit to sample 2 experimental concentrations. The simulation model included cartilage diffusivity and bone resistivity. 48

Illustration 21 Simulated best fit to sample 2 experimental concentrations. Bone resistivity was not included in the simulation model. 48

Illustration 22 Fluorescent Microscope image of an unexposed sample 61

Illustration 23 Sample 1 exposed to FITC FD4 for 17 hours 62 Illustration 24 Sample 10 cartilage exposed directly to Fluorescein FD4 for 98 hours ... 63 Illustration $25 \mathrm{Gd}$ concentration at various times, throughout the cartilage depth for all sample 1

Illustration $26 \mathrm{Gd}$ concentration at various times, throughout the cartilage depth for all sample 2 80

Illustration $27 \mathrm{Gd}$ concentration at various times, throughout the cartilage depth for all sample 4 .....

Illustration $28 \mathrm{Gd}$ concentration at various times, throughout the cartilage depth for all sample 3

Illustration $29 \mathrm{Gd}$ concentration at various times, throughout the cartilage depth for all sample 6

Illustration $30 \mathrm{Gd}$ concentration at various times, throughout the cartilage depth for all sample 5

Illustration $31 \mathrm{Gd}$ concentration at various times, throughout the cartilage depth for all sample 7 83

Illustration 32 Sample 1: simulated fit to experimental concentrations 84

Illustration 33 Sample 2: simulated fit to experimental concentrations 84 
Illustration 34 Sample 3: simulated fit to experimental concentrations 85

Illustration 35 Sample 4: simulated fit to experimental concentrations 85

Illustration 36 Sample 5: simulated fit to experimental concentrations ........................ 86

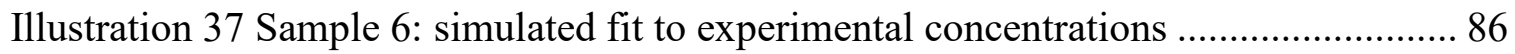

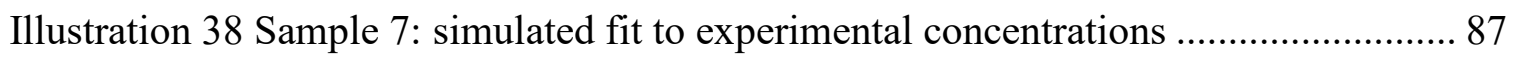

Illustration 39 Sample 1: simulated fit to experimental concentrations, without the bone barrier. 88

Illustration 40 Sample 2: simulated fit to experimental concentrations, without the bone barrier. 89

Illustration 41 Sample 3: simulated fit to experimental concentrations, without the bone barrier..... 89

Illustration 42 Sample 4: simulated fit to experimental concentrations, without the bone barrier. 90

Illustration 43 Sample 5: simulated fit to experimental concentrations, without the bone barrier..... 90

Illustration 44 Sample 6: simulated fit to experimental concentrations, without the bone barrier. 90

Illustration 45 Sample 7: simulated fit to experimental concentrations, without the bone barrier. 


\section{List of Appendices}

Appendix A - Diffusion chamber engineering drawing ............................................. 79

Appendix B - Experimental Gd concentration at select cartilage depths and times for all

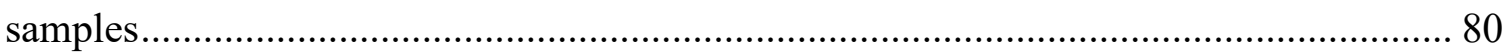

Appendix C - Simulated Gd concentration fit to experimental results at select cartilage

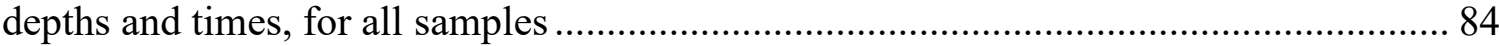

Appendix D - Simulated Gd concentration without the bone barrier fit to experimental results at select cartilage depths and times, for all samples ...................................... 88 


\section{Acronyms}

\begin{tabular}{|c|c|}
\hline cAMP & Cyclic adenosine monophosphate \\
\hline $\mathrm{D}$ & Diffusivity \\
\hline $\mathrm{ECM}$ & Extracellular matrix \\
\hline FD & Finite difference \\
\hline GAG & Glycosaminoglycans \\
\hline $\mathrm{Gd}$ & Gadobutrol \\
\hline $\mathrm{HGF}$ & Hepatocyte growth factor \\
\hline IGF & Insulin-like growth factor \\
\hline IL & Interleukin \\
\hline MMP & matrix metalloproteinase- 13 \\
\hline MOAKS & MRI osteoarthritis knee score \\
\hline MR & Magnetic resonance \\
\hline MRI & Magnetic resonance imaging \\
\hline MW & Molecular weight \\
\hline $\mathrm{OA}$ & Osteoarthritis \\
\hline $\mathrm{OCT}$ & Optimal cutting temperature \\
\hline OPG & Osteoprotegerin \\
\hline PAR & Proteinase-activated receptor \\
\hline PTH & Parathyroid hormone \\
\hline SOST & Sclerostin \\
\hline RANK & Receptor activator of nuclear factor $\kappa \mathrm{B}$ \\
\hline RANKL & Receptor activator of nuclear factor $\kappa$ B ligand \\
\hline
\end{tabular}




$\begin{array}{ll}\text { RF } & \text { Radio frequency } \\ \text { RFU } & \text { Relative florescence units } \\ \text { RMS } & \text { Root-mean-square } \\ \text { ROI } & \text { Region of interest } \\ \text { SHOMRI } & \text { Scoring hip osteoarthritis with MRI } \\ \text { T1 } & \text { MRI Longitudinal relaxation time } \\ \text { T2 } & \text { MRI Transverse relaxation time } \\ \text { TGF } & \text { Transforming growth factor } \\ \text { TNF } & \text { Tumor necrosis factor } \\ \text { 1D } & \text { One-dimension }\end{array}$




\section{Nomenclature}

\begin{tabular}{|c|c|}
\hline $\mathrm{B}_{0}$ & Applied magnetic field, tesla (T) \\
\hline $\mathrm{C}$ & Concentration, $\left(\mathrm{mMol} / \mathrm{cm}^{3}\right)$ \\
\hline $\mathrm{D}$ & Diffusivity, $\left(\mathrm{cm}^{2} / \mathrm{s}\right)$ \\
\hline I & Intensity, $\left(\mathrm{cd} / \mathrm{m}^{2}\right)$ \\
\hline M & Magnetic Moment, $(\mathrm{Nm} / \mathrm{T})$ \\
\hline Ux & Flow velocity, $(\mathrm{cm} / \mathrm{s})$ \\
\hline $\mathrm{R}$ & Resistivity, $\left(\mathrm{s}^{-1}\right)$ \\
\hline $\mathrm{r}_{1}$ & Longitudinal relaxivity, $(\mathrm{mMol} \cdot \mathrm{ms})^{-1}$ \\
\hline$r_{2}$ & Transverse relaxivity, $(\mathrm{mMol} \cdot \mathrm{ms})^{-1}$ \\
\hline $\mathrm{S}$ & Signal, (dimensionless) \\
\hline $\mathrm{t}$ & Time, (s) \\
\hline $\mathrm{T}_{1}$ & Longitudinal relaxation rate, $(\mathrm{ms})^{-1}$ \\
\hline $\mathrm{T}_{2}$ & Transverse relaxation rate, $(\mathrm{ms})^{-1}$ \\
\hline $\mathrm{x}$ & Distance from osteochondral junction, $(\mathrm{cm})$ \\
\hline$\Gamma$ & Flux, $\left(\mathrm{mol} / \mathrm{m}^{2} \mathrm{~s}\right)$ \\
\hline$\rho$ & Density, $\left(\mathrm{g} / \mathrm{cm}^{3}\right)$ \\
\hline$\varnothing$ & Porosity, (dimensionless) \\
\hline
\end{tabular}




\section{Chapter: Introduction}

As medicine has continued to advance, the population continues to age and with this comes its own challenges. One of the most common health challenges medicine is faced with when it comes to the aging population, is osteoarthritis (OA). OA is the most common form of arthritis, affecting $13 \%$ of the Canadian population (4.6 million Canadians) and over 27 million people worldwide [1-3]. The costs associated to debilitating OA can be hard to measure as they not only include individual financial burden and economic burden on the health care system but a variety of personal burden to those affected [4]. Considering the prevalence of this disease, as well as the burden it poses on individuals and society as a whole, it is a disease that deserves great attention.

Osteoarthritis was long seen as a disease of the cartilage, however with more recent findings on the effects of $\mathrm{OA}$ on other joint components, understanding the interaction between components is essential to better understanding OA [5]. A joint's major components are bones, cartilage, ligaments, tendons, muscles and synovial fluid. Each of these components plays a very specific part in maintaining a functional and healthy joint [6]. Articular cartilage is a very specialized tissue covering articulating bones in the body. This tissue allows nearly frictionless motion between bones and can withstand large loads [7]. When there are disturbances in the cartilage composition, the function of the cartilage is compromised. OA is a disease that has differing effects on each of the joint components, however, in its final stages, the cartilage can be severely degenerated or entirely eroded from the underlying bone [8]. This makes for very difficult day-to-day movements of the affected joint(s) and can have severe consequences on quality of life [4, 9-10]. 
Although many tissues in the joint may undergo changes with OA degeneration, the more severely affected components are cartilage, bone and synovial fluid.

\subsection{Articular Cartilage}

Cartilage is a type of highly specialized connective tissue [11]. There are three categories of cartilage: hyaline, elastic and fibroelastic. Each has its own role in the body and has different composition and arrangement of cells and fibers [11]. Articular cartilage is a type of hyaline cartilage and can be found in joints and serves as an interface between bones, distributing load, providing frictionless motion between bones and protecting the bone from wearing [12]. The average thickness of articular cartilage in the knee ranges from 1.69 to $2.55 \mathrm{~mm}$ and 1.35 to $2.0 \mathrm{~mm}$ in the hip [13]. Although articular cartilage is avascular, aneural and alymphatic it is essential that the cells within cartilage, called chondrocytes, receive nutrients and oxygen to maintain tissue function [12, 14]. Transport of nutrients allows a number of functions to occur in articular cartilage: synthesis of collagen, proteoglycans and glycosaminoglycans $[12,15]$.

\subsubsection{Composition and Structure of Articular Cartilage}

Cartilage is a multiphasic tissue comprised of a fluid phase and solid phase [6]. It is also a viscoelastic tissue, meaning that it responds to loads in a time dependent manner [6]. It is primarily made up of extracellular matrix which consists of water, proteoglycans and collagen $[12,16]$. Collagen makes up approximately $60 \%$ of the solid phase of cartilage and forms a very complex, but organized system [17]. Type II collagen is the predominant type of collagen and makes up for approximately $90-95 \%$ of the collagen found in cartilage 
[6]. Collagen contributes to cartilage's tensile stiffness and, sheer and compressive properties [16]. The proteoglycans provide the tissue with its osmotic properties, which in turn plays a major part in cartilage's biomechanical properties and behavior [17]. The flow of fluid in cartilage is also believed to be responsible for the flow of nutrients and other components necessary for maintaining homeostasis [6]. Chondrocytes, the cells in cartilage, are responsible for the synthesis of the above mentioned macromolecules and also maintain the protein network that makes up the tissue, known as the extra-cellular matrix (ECM) [16]. The chondrocytes are dispersed throughout the ECM and receive nutrients via the flow of water in and out of the ECM [17]. It is known the chondrocytes interact with the synovial fluid [14]. Multiple factors affect chondrocytes, including mechanical injury to the tissue. Injury to cartilage decreases chondrocyte activity and can also induce the production of pro-catabolic molecules such as interleukins [52]. Other factors that affect chondrocyte activity are age and their physiochemical and mechanical environment.

The differing organization of proteoglycans, collagen and chondrocyte throughout the cartilage is what divides the cartilage into three different zones: deep zone, middle zone (transitional zone) and superficial zone (Illustration 1) $[15,16]$. The vertically-oriented collagen fibers in the deep zone insert into a layer of calcified cartilage, anchoring the cartilage to the bone. In the middle zone, the fibers are less organized and appear to be oriented randomly, which allows for swelling and depressing. Finally, in the superficial zone, the fibers are organized parallel to the cartilage surface and are also denser. The interface between the calcified and non-calcified cartilage is known as the tidemark. The 
tidemark is visible and can be seen in histological sections. The calcified cartilage zone is where the cartilage transitions into bone.

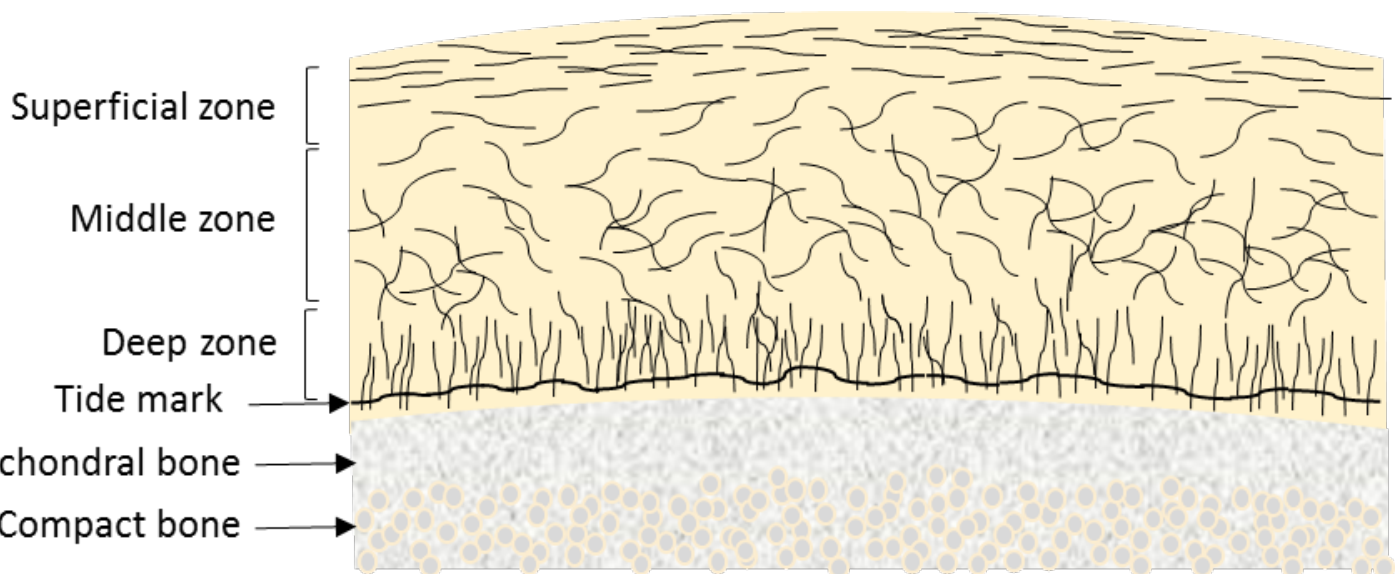

Illustration 1 Articular cartilage zones: superficial, middle and deep

Proteoglycans are composed of glycosaminoglycans (GAGs) linked together by a protein core [16]. GAGs are highly negatively charged and thus attract positively charged ions (cations). Because water is a polar molecule, its positively charged hydrogen atoms are attracted to the GAGs. Proteoglycans also bound to each other to form large negativelycharged aggregates which provides the osmotic swelling properties of cartilage [16]. The proteoglycan concentration in cartilage is homogeneous, but rather the concentration increases near the bone [15].

\subsubsection{Properties of Articular Cartilage}

Cartilage is an adaptable tissue with unique properties. The compressive stiffness and shear stiffness are largely attributed to the osmotic swelling properties of cartilage caused by the negatively charged GAGs. Since the GAGs attract water molecules this causes swelling of the ECM, which in turn leads to swelling of the cartilage. The imbalance 
of ions between the interstitial fluid and the external environment contributes to the swelling pressure $[6,18]$. When cartilage is loaded, the movement of interstitial fluid along with high frictional drag forces between this fluid and the solid phase, and the pressure in the fluid phase, give rise to time-dependent poroelastic behavior [6]. This behavior allows the cartilage to respond appropriately to compressive loads and strain [6]. Once load or strain is removed, cartilage returns to its state of equilibrium [6]. Collagen fibrils and proteoglycans entangled within the collagen give rise to cartilage tensile properties, preventing deformation of the tissues [6]. Articular cartilage structure allows it to store, dissipate, and transmit mechanical energy [19].

\subsection{Subchondral Bone}

Subchondral bone is the bone lying immediately under the articular cartilage [20]. The subchondral bone is made up of trabecular bone and compact bone. Cartilage is anchored to the bone via collagen fibers. Unlike cartilage, bone, on the other hand, has both blood vessels and nerves. The blood flow brings nutrients and oxygen to the bone. It has been shown that subchondral bone plays an important role in absorbing shock and supporting the joint [22]. For many years, cartilage was the focal point of OA research, but the focus has shifted and subchondral bone has gained much attention [20]. Thanks to radiographic imaging, it can be confirmed that the first visible change of OA occurs in the subchondral bone $[21,22]$, and the first detectable biochemical changes also occur in the bone [23]. In the early stages of OA it can be seen that the subchondral bone, both trabecular and compact, are modified and experience changes in its bone remodeling cycle [24]. Sclerosis, osteophyte formation and bone remodeling leading to thickening of the 
bone plate are the symptoms of OA seen in the bone $[24,25]$. MRI has also demonstrated that OA affected bone marrow experiences changes such as edema and bone cysts [24]. Although, it was long believed that the osteochondral junction was impermeable to solute transportation, it has been confirmed that presence of subchondral bone in cartilage tissue culture has a direct effect on the survival of chondrocytes found in articular cartilage [14]. However, the mechanism involved has not yet been identified. This discovery along with the effects of OA on the joint, puts into question the impermeability of the osteochondral junction. Further to this, it has been proven that calcified cartilage is permeable to small, nutrient size solutes coming from the subchondral bone [26].

\subsection{Osteoarthritis: A Disease of the Joint}

Osteoarthritis is an idiopathic, progressive and disabling joint disease that affects millions of people around the world [3]. Many factors affect OA and its progression, making it a hard to predict disease. OA was often believed to be a mechanical disease until the $21^{\text {st }}$ century when the scientific community started to look at the joint as an organ and how OA affects the entire organ, not just the cartilage $[25,27]$. OA has more than just one tissue determining its progression; cartilage, subchondral bone and the synovial membrane all play their part in the disease [25]. It has been established that the synovial membrane inflammation plays an active role in the progression of the disease [28]. The mechanism of degradation remains unknown [29]. OA is characterized by reduced function of the joint as well as diminished metabolic homeostasis, meaning that the metabolic activities in the joint are abnormal. Although OA can occur in all articular joints, it is most common in the spine, knee and hip joints [30-31]. Severe and debilitating pain are not uncommon in 
affected joints, resulting in limited function of the joint and can lead to major impacts on the quality of life for those affected [4, 32-34]. It is a disease that has been documented to have affected humans for thousands of years [38]. Genetic factors also became recognized as a plausible cause of osteoarthritis and the medical community started to shift its focus $[29,34]$. Subchondral bone resorption is one of the first visible and diagnosable signs of OA seen through medical imaging [35]. The change in subchondral bone activity leading to bone sclerosis occurs in the early stage of the disease [28]. According to Wolff's law, bone structure will adapt to the loads under which it is placed [94]. This means that high loads will cause bone remodeling, which in turn could also lead to cartilage damage. The later stages of OA are marked by the sclerosis of the bone and active bone remodeling leading to thickening of the subchondral bone plate [36]. Overall, dysfunctional bone

metabolism could be seen by a few other indicators observed in the later stage OA: osteocalcin and osteopontin in the synovial fluid, elevated levels of growth factors such as transforming growth factor and insulin-like growth factor [28,37]. Osteocalcin is a marker of bone formation, while osteopontin is a bone matrix protein [28].

\subsubsection{Etiology}

Although OA remains an idiopathic disease, there are multiple indicators of OA [14]. Some of the main factors include age, obesity, gender, genetic predispositions, joint deformities and injury, among others [25,39-40]. Mechanical factors were believed to be the only cause of osteoarthritis for many centuries, however in the $21^{\text {st }}$ century, studies have begun to focus on genetic factors that may be leading to OA [3, 29]. Studies have 
demonstrated that as many as $39-79 \%$ of OA cases can be attributed to genetic factors $[3$, $5,58]$.

The most prevalent risk factor of OA is age [3]. It is not yet understood why this influences the chances of getting OA but a clear relationship between aging and OA has been identified $[42,43]$. This is especially concerning as the population continues to get older. While age is the most prevalent risk factor of OA, age and OA are inter-related but not inter-dependent [44]. Obesity is also a risk factor, increasing chances of getting OA in the hip moderately, but increases chances significantly of getting OA in the knee joints [45, 46]. Gender has also been identified as a risk factor, especially when it concerns OA in the knee. Being female increases the chances of getting knee OA significantly $[47,48]$. It has even been reported that while $10 \%$ of men over the age of 60 are affected, $18 \%$ of women over 60 are affected [29]. The reason for this could be due to several factors, including differences in hormones, biochemical differences and differences in joint sizes [47]. A variety of injuries to tendons, ligaments, cartilage, and meniscus have demonstrated a direct link to development of osteoarthritis in the knee and hip joints [49]. Although not an acute injury, but rather a chronic factor causing injury over time, joint malalignment has shown to be a direct cause for OA in both knee and hip joints [50-51].

\subsubsection{Diagnosis}

OA is a disease that manifests slowly, in the scale of years. This disease is typically accompanied by chronic pain in two forms: an intermittent but severe pain and a persistent aching [32]. OA is diagnosed based on symptoms, clinical examination and radiographic imaging. Symptoms may include swelling, pain, stiffness, loss of range of motion and 
tenderness. Malalignment, swelling and palpable effusions are symptoms that can be identified by clinicians during examination. Radiographically, clinicians can diagnose OA based on narrowing of the joint space, sclerosis i.e. increased density of the subchondral bone, and osteophytes on the subchondral bone $[8,29]$.

Imaging has been key in diagnosing $\mathrm{OA}$ and confirming that $\mathrm{OA}$ is not only a cartilage disease, but a disease of the joint. To better understand OA, it is crucial to better understand the relationship between components. This has been a challenge for clinicians and researchers as it is difficult to conduct long term experiments in vivo and studying the joint and its ecosystem in vitro is nearly impossible as the joint will lose much of its functionality as soon as it is excised from the body.

As mentioned, radiographic imaging has not only led to the discovery that OA affects other components of the joint, but it has also become the standard diagnostic tool for OA. Currently, clinicians can diagnose OA based on symptoms and x-rays of the affected joint. Although x-rays have played an important role in furthering knowledge of OA, it has its limitations, mainly that it does not image soft tissue. A grading system was developed in 1957 by Drs. Kellgren and Lawrence and was accepted by the World Health Organization (WHO) in 1961 [8]. The grading system is reliant on radiographic images of the joint and often requires multiple images [8]. The Kellgren-Lawrence grading scale has 4 grades, with 4 being the most severe stage of OA. The scale considers the joint space, the bone density and osteophytes, three symptoms that can only be seen in radiographic images. Unfortunately, these symptoms are only found in the later stages of OA. For reasons yet to be fully understood, these radiographically diagnosed symptoms do not correlate with patient reported symptoms [31]. Radiography is limited in evaluating the 
joint as it does not image cartilage and is often unable to assist in diagnosing early stage

OA.

Table 1 Kellgren-Lawrence OA grading scale [8]

\section{Grade}

1 Doubtful narrowing of the joint space and possible osteophytic lipping

2 Definite osteophytes and possible narrowing of the joint space

3 Moderate multiple osteophytes, definite narrowing of the joint space and some sclerosis, and possible deformity of bone ends

$4 \quad$ Large osteophytes, marked narrowing of joint space, severe sclerosis and definite deformity of bone ends

Magnetic resonance imaging (MRI) is an imaging modality that captures an image of soft tissues, such as cartilage. Unfortunately, standard MRI techniques do not allow for high enough resolution to obtain a good image of the cartilage [35]. In recent years, MRI capabilities have improved thanks to contrast enhanced imaging and higher magnetic fields. Unfortunately, MRI is not systematically used as a diagnostic tool for OA because of its high cost, long acquisition times and poor spatial resolution [35]. Some grading systems for MRI diagnosis such as MRI Osteoarthritis Knee Score (MOAKS) and Scoring Hip Osteoarthritis with MRI (SHOMRI) have started to emerge within the last decade [60, 71]. MRI has interesting potential, for example the ability to quantify damage to the collagen network [63]. Contrast enhanced MRI first was introduced in 1988 and has since been proven to produce better images for various tissues and their diseases [64]. This MRI technique has become increasingly popular and has shown great potential for better MR image acquisition for OA diagnosis. In the case of OA, cartilage has depleted amounts proteoglycans and allows for more contrast agent to diffuse through when compared to healthy cartilage, especially when negatively charged compounds are used [65, 66]. Further, MRI scanners with much larger magnetic fields have been developed and are being 
used for clinical research [72]. Higher magnetic fields lead to better signal-to-noise ratios, higher resolution and also faster imaging times, but these scanners come at an even larger cost $[69,74]$. These improvements to MRI could be employed to detect early stage OA and monitor the progression of the disease.

\subsubsection{Treatment}

Treating OA is very limited due to the limited abilities of cartilage to repair itself and the lack of understanding of the disease itself. The current focus for clinicians is to prevent OA progression and to maintain as best of a quality of life as possible. There is no real cure for osteoarthritis, however the symptoms are most often treated with pharmaceuticals, therapies, assistive devices, and in many cases by total joint replacement (arthroplasty) as the final solution $[33,67]$. Since this final option is often viewed as the last possible solution and the disease is a slow manifesting one, many people must live with their pain and limited mobility for years. Disability due to OA has a profound impact on the quality of life for patients [69-71]. Because of the disease longevity, it brings a heavy economic burden to sufferers as well as the health care system [4].

Currently, pharmaceuticals are only effective for treatment of symptoms such as inflammation and pain. Thus, there have been no successful pharmaceuticals for stopping or reversing OA [73].

In the cases where patients are overweight, it is often recommended to lose weight as this can relieve some of the stress on the joint and consequentially some of the pain [74]. This also helps to delay the progression of OA. In cases where OA is not caused by injury, 
light to moderate exercise has been proven to delay the progression of OA and in some cases, some relief of pain $[75,76]$.

Osteoarthritis is one of the most common indications for arthroplasty of the hip and knee. To qualify for arthroplasty, patients must be in the later stages of OA [77]. Arthroplasties are not always guaranteed to relieve all pain and in some cases fail due to mechanical wear of components, leaving the patient with equal or worse pain but is becoming more common practice as it is successful in many instances [32]. Surgically, patients may also undergo debridement and lavage of the joint in an attempt to smooth the rougher portions of the cartilage, thus improving mobility of the joint [78].

\subsubsection{Factors and Biomarkers in Osteoarthritis}

In the case of the non-injury induced OA, the study of factors and biomarkers, i.e. biochemical compounds found in the joint in the presence of OA, is essential to better understanding the pathogenesis. Biomarkers are used to indicate abnormal biological processes, in this case slow degeneration of cartilage. Unfortunately, few biomarkers have been validated for the purpose of diagnosing OA $[28,52]$. Multiple studies have been undertaken to observe specific biomarkers and cells found in the subchondral bone that were seen to have an increased or decreased presence in joints affected by OA. Biochemical communication between tissues, known as crosstalk, may be an important mechanism in cartilage metabolism, but thus far has been largely ignored. It is also possible that pharmaceuticals have been unsuccessful thus far because they only target cartilage and not the osteochondral joint as a whole and the crosstalk taking place between joint components. 
One grouping of biomarkers can be classified as cytokines. Cytokine molecules are essential in mediating cellular interactions with other cells [53]. Further to this, cytokines are responsible for regulating chondrocyte function [53]. Catabolic cytokines are defined as cytokines that directly or indirectly induce the breakdown of cartilage ECM, while anabolic cytokines can be seen as cytokines that inhibit the activity of catabolic cytokines as well as increase synthetic activity of chondrocytes [53, 54]. Cytokines rarely act alone in their biological functions, but rather act with multiple other cytokines [53]. Cytokines of particular interest in OA include interleukins (IL), tumor necrosis factor (TNF), insulin-like growth factor (IGF), transforming growth factor (TFG), receptor activator of nuclear factor (RANK), RANK ligand (RANKL) and osteoprotegerin (OPG) $[28,53]$. Ephrin ligands control bone remodeling and play a role in abnormal metabolism of OA [28]. Chondrocytes in OA have been shown to produce OPG, RANK and RANKL subsequently resulting in increased levels of matrix metalloproteinase-13 (MMP) an enzyme involved in destruction of cartilage ECM, and proteinase-activated receptor-2 (PAR-2) which are both involved in the degenerative process of OA in cartilage [55]. Also of interest is the parathyroid hormone (PTH) which stimulates bone osteoblasts and leads to less production of cyclic adenosine monophosphate (cAMP) [28]. OA subchondral bone osteoblasts have also demonstrated reduced PTH receptors [56, 57]. The hepatocyte growth factor (HGF) is also considered a biomarker due to its elevated levels and is associated with crosstalk between bone and cartilage in OA [58-60]. HGF also induces MMP-13 [28, 58]. Production of sclerostin (SOST) and Wnt antagonist directly leads to osteocyte bone resorption $[28,61]$. For simplicity, these factors and biomarkers are identified in table 2: 
Table 2 Biomarkers and indicative factors of osteoarthrtitis [13, 64-70]

\begin{tabular}{|c|c|c|}
\hline $\begin{array}{l}\text { BIOMARKER/ } \\
\text { FACTOR }\end{array}$ & $\begin{array}{l}\text { MOLECULAR } \\
\text { WEIGHT (kDa) }\end{array}$ & $\begin{array}{l}\text { TYPE OF } \\
\text { INTERACTION }\end{array}$ \\
\hline IL-1 $\beta$ & 17.5 & Catabolic \\
\hline IL-6 & 21 & Modulatory \\
\hline TNF $\alpha$ & 26 & Catabolic \\
\hline IGF-1 & 7.65 & Anabolic \\
\hline TGF- $\beta 1$ & 25 & Anabolic \\
\hline RANKL & 35 & Catabolic \\
\hline OPG & $\begin{array}{l}60 \text { (monomer) } \\
120 \text { (dimer) }\end{array}$ & Catabolic \\
\hline MMP-13 & 60 & Catabolic \\
\hline PAR-2 & 44 & Catabolic \\
\hline PTH & 9.4 & Anabolic \\
\hline HGF/NK1, NK2 & 60 & Catabolic \\
\hline SOST & 23.3 & Anti-anabolic \\
\hline CAMP & 0.33 & Modulatory \\
\hline
\end{tabular}

Since osteoblasts (bone) and chondrocytes (cartilage) are cells derived from the mesenchymal stem cell line, they produce and respond to many of the same biomarkers, suggesting a role for crosstalk between tissues in both healthy and degenerated joints. 


\subsection{Hypotheses}

The purpose of this research is to investigate the osteochondral junction and its permeability to further our understanding of the relationship between bone and cartilage. Investigating through various methods to measure diffusion will provide a more holistic understanding of the osteochondral crosstalk. More specifically, in this study we hypothesize:

1. molecular diffusion across the osteochondral junction can be measured using contrast-enhanced MR imaging;

2. the osteochondral junction represents a barrier to molecule diffusion that may be measured by a resistivity; and,

3. large molecules (MW up to $70 \mathrm{kDa}$ ) can diffuse across the osteochondral junction, suggesting a biochemical crosstalk between bone and cartilage, and that this changes with OA degeneration. 


\section{Chapter: Diffusivity Assessment Across the Osteochondral Junction}

\section{Using Contrast Enhanced MRI}

\subsection{Introduction}

Currently, the standard for clinical imaging and diagnosing patients with osteoarthritis is X-ray imaging. In fact, the definition of OA is based on radiography [91]. Although X-rays have been very helpful in identifying diseased bone and bone fractures in a non-invasive way, X-rays are not able to image cartilage which is crucial in better understanding this disease $[35,91,92]$. Although radiographic imaging can detect changes in the subchondral bone and narrowing of the joint space, it is reported that a reduction of $11-13 \%$ of cartilage volume is necessary before observing any changes [79]. Meanwhile, MRI has been reported as a superior method at detecting early osteoarthritis, as it can image changes in the joint space for reductions in volume as little as $2 \%$ [79]. MRI also provides images of the bone, and can provide images of soft tissues, giving a much more complete image of the joint, which can be crucial in diagnosing and monitoring the progression of OA [93]. MRI is not only crucial in diagnosis; it can also play a very important role in better understanding the crosstalk between bone and joint by observing the diffusion of solutes into and out of the bone. The osteochondral junction has recently been established as being permeable to smaller molecules [26]. It is also known that ions create a concentration gradient which drives diffusion [26]. Diffusion is also influenced by steric interactions between the solution and the solid matrix [82].

Gadolinium is a contrast agent frequently employed in compounds of MRI contrast agents such as gadobutrol [100]. Gadobutrol has a molecular weight of $605 \mathrm{Da}$ which is considerably small and can diffuse into various tissues in the body [100]. This size of 
molecule is considered to be small and representative of nutrient molecules. The hypothesis of this study is that molecular diffusion across the osteochondral junction can be measured using contrast-enhanced MRI. Diffusing gadobutrol across the osteochondral junction would confirm that neutrally-charged low molecular weight contrast agent molecules can passively diffuse across, which could be useful for studying changes in subchondral bone permeability in vivo. To quantify the diffusion of adobutrol, the images obtained will provide intensity values which can be converted to concentration values. The concentration values will also provide more information on the diffusion properties of cartilage. Understanding the diffusion and dispersion of small, nutrient-sized solutes will help to better understand cartilage metabolism. Furthermore, observing this through 7 Tesla MRI permits image acquisition with a very high spatial resolution.

\subsubsection{Magnetic Resonance Imaging}

Magnetic resonance imaging is an imaging modality that takes advantage of the magnetic moment of hydrogen atoms, typically those found in water or in fat tissue. Because the human body has a high-water composition, especially in softer tissues, this is ideal for MR imaging [97].

Charged particles, such as hydrogen in water, have a small directional magnetic moment due to their magnetic dipoles. When a large static magnetic field $\left(\mathrm{B}_{0}\right)$ is applied to these charged particles, their nuclei align themselves in the direction of the applied magnetic field, causing their directional magnetic field to be parallel to $\mathrm{B}_{0}$ at equilibrium [97]. This direction is conventionally called the z-axis. The magnetization of a tissue sample in $\mathrm{B}_{0}$ is the vector sum of the magnetic moments, called M (Illustration 2). 


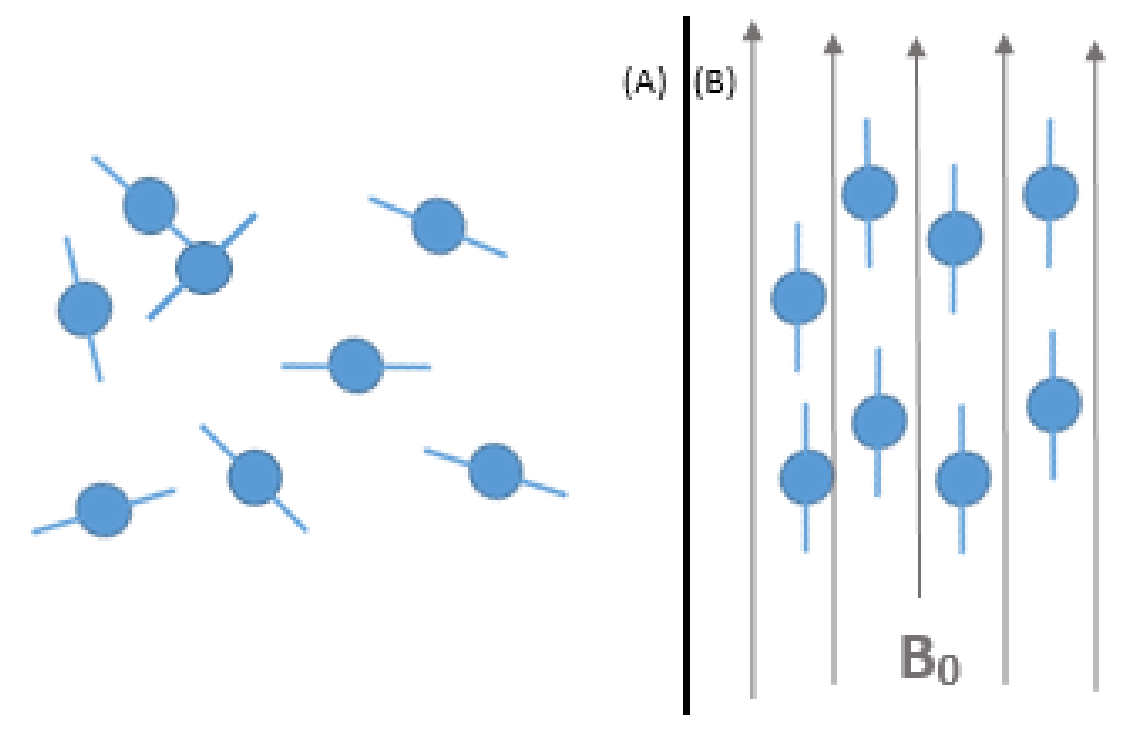

Illustration 2 (A) Hydrogen atoms with the axis representing the magnetic moment, M, without $B_{0}$ (B) Hydrogen atoms aligned with applied $B_{0}$

A radio-frequency $(\mathrm{RF})$ pulse is then applied perpendicular to $\mathrm{B}_{0}$ and $\mathrm{M}$ which causes $\mathrm{M}$ to tilt away from $\mathrm{B}_{0}$ and the $\mathrm{z}$ axis towards the $\mathrm{XY}$ plane and at the same time precessing about the $\mathrm{Z}$ axis [97]. As $\mathrm{M}$ rotates and passes through a conducting coil it induces a measurable voltage according to Faraday's Law [98]. When the RF pulse stops, $\mathrm{M}$ realigns with $\mathrm{B}_{0}$ this is known as relaxation. During relaxation, the transverse component of $\mathrm{M}$ decreases and the longitudinal component increases, however these phenomena are independent. The decrease in the transverse component is measured as a voltage signal decrease that is captured by the coil surrounding the body being imaged [97]. This voltage potential is then processed to produce an image. Each image consists of voxels, representing a small volume within the tissue on the order of $1 \mathrm{~mm}^{3}$. The image intensity of the voxel is proportional to the proton density of the tissue [97]. The more 
hydrogen atoms present, the higher the proton density which giver a higher voxel intensity [97].

Contrast in MR images is also dependent on two time constants known as T1 and T2. $\mathrm{T} 1$ is known as the longitudinal relaxation time, and is exponential recovery time constant governing the time required for the nuclei to return to its alignment with $\mathrm{B}_{0}$ [97] (Illustration 2). T2 is known as the transverse relaxation time and is the exponential decay time constant that measures the time required for the nuclei to fall out of phase as they spin in the plane perpendicular to B0, reducing the transverse component of M [97].

Contrast enhanced MR imaging is a method employed to obtain physiological or compositional information about tissues. In MR imaging this is achieved by introducing a paramagnetic element such as gadolinium [99]. Paramagnetic elements slightly increase the local magnetic field to shorten recovery time (T1) [99]. Thus, T1 will be lower in regions containing gadolinium. Using MR image acquisition sequences sensitive to T1 i.e. T1-weighted imaging, can thus be used to assess the distribution of gadolinium as it diffuses through a tissue sample.

The goal of this study is thus to assess diffusion of small molecules from bone into cartilage using contrast-enhanced MRI. 


\subsection{Methods}

\subsubsection{Diffusion Chamber Design}

Custom diffusion chambers were machined for these studies (Illustrations 3 and 4). To be able to use the chambers in an MRI scanner, no metal could be employed and so Delrin was chosen. Delrin, or polyoxymethylene, is a type of thermoplastic. Plastic washers with thickness of $2.5 \mathrm{~mm}$, inner diameter of $10 \mathrm{~mm}$, were chosen to fit the $10 \mathrm{~mm}$ sample diameter, and outer diameters of $22 \mathrm{~mm}$. The diffusion chamber was then designed to have an inner diameter of $22 \mathrm{~mm}$ through the first chamber and an inner diameter of $15 \mathrm{~mm}$ for the second chamber. Because of the change in diameter, this created a lip where the washers could sit in place, creating the division between chambers. For the sake of simplicity, the outer diameter of the entire chamber and thread pitch were made to be that of a typical garden hose, so that stoppers found at the hardware store could be used to seal the chamber. Engineering drawings of the chamber are included in Appendix A.

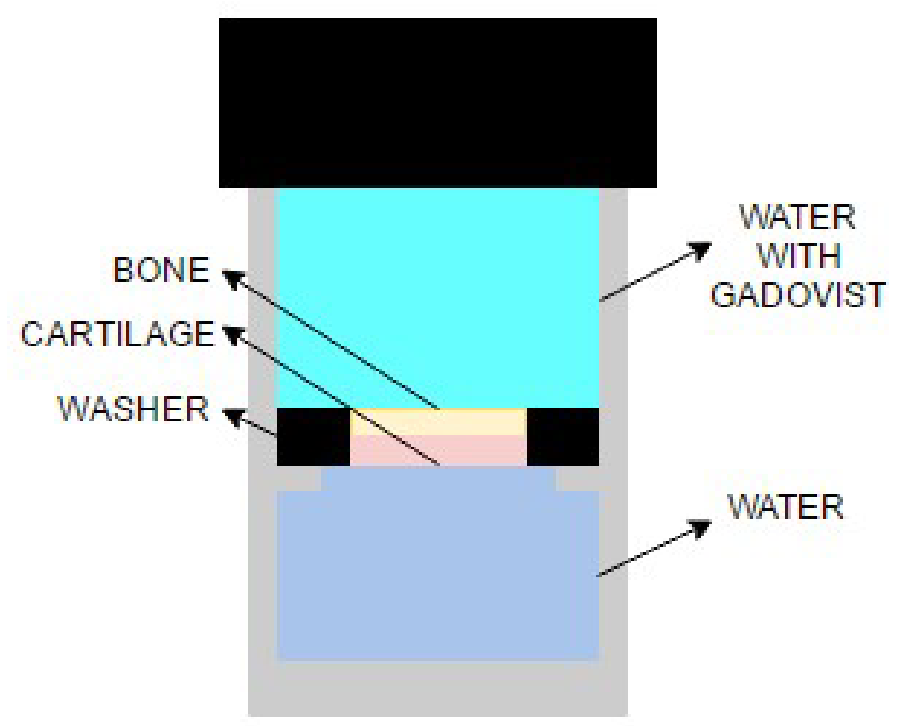

Illustration 3 Diffusion chamber model 


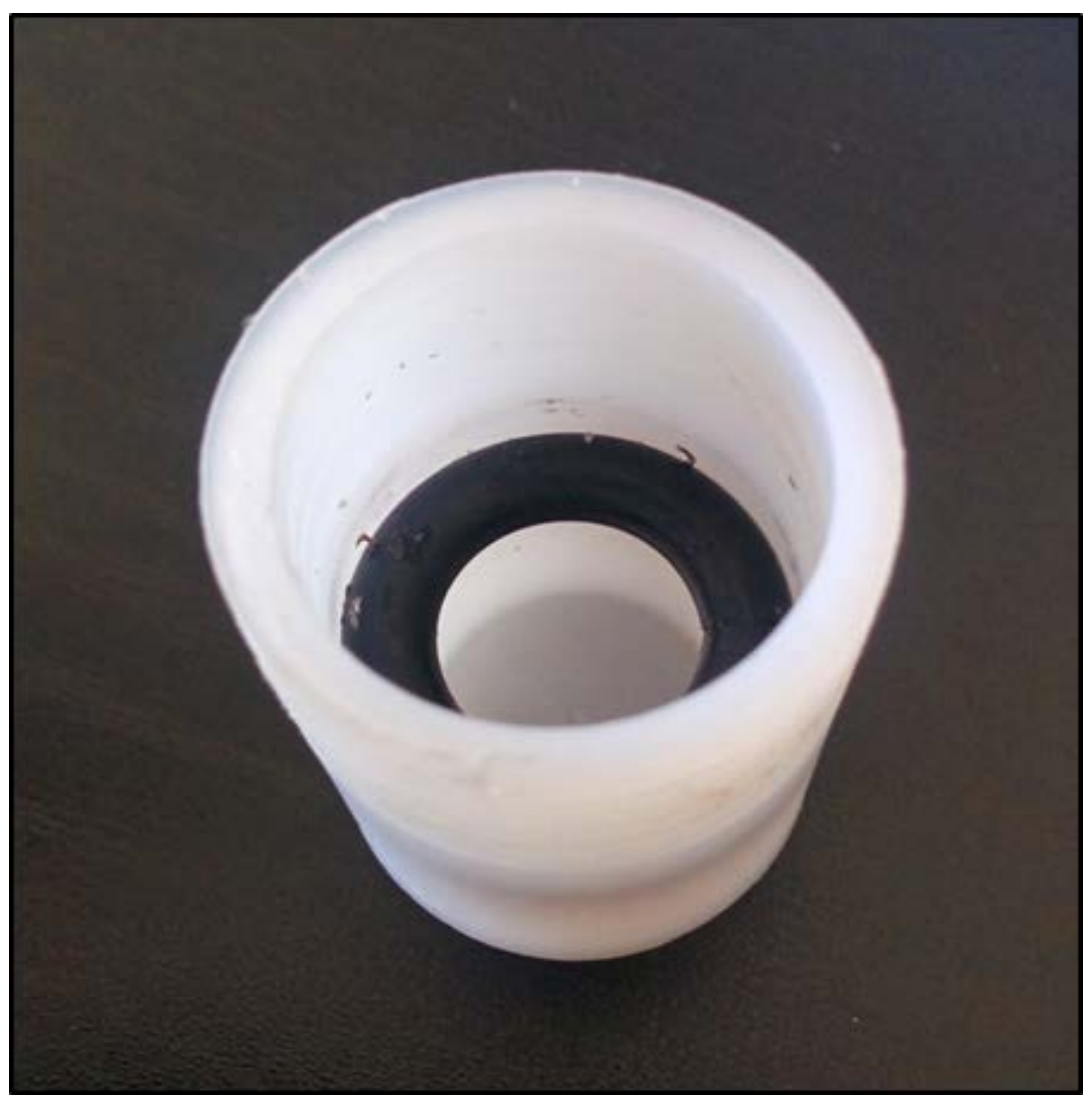

Illustration 4 Purpose-made diffusion chamber

\subsubsection{Sample Preparation}

Frozen porcine femurs with little to no damage were obtained from a local slaughterhouse. The joints are often taken from young animals and had no visible signs of $\mathrm{OA}$ or trauma and were kept frozen at an approximate temperature of $-18{ }^{\circ} \mathrm{C}$ until the MRI schedule permitted for 40-60 hours of continuous imaging. After thawing the femurs, osteochondral plugs were cut out of the lateral and medial condyles using a surgical trephine (hole saw). Only the plugs that showed a consistent cartilage thickness were chosen for testing. The samples were mostly taken from the areas of high contact. In some joints, multiple samples could be obtained and these samples were considered to be independent. Each plug was cut to have a diameter of $10 \mathrm{~mm}$ and a thickness varying 
between 4 and $5 \mathrm{~mm}$ depending on the thickness of the cartilage (Illustration 5). The cartilage was left at its natural thickness and the bone was cut to be no more than $3 \mathrm{~mm}$ to reduce diffusion time. It was not required for the plugs to be fixed in formalin or other fixative.

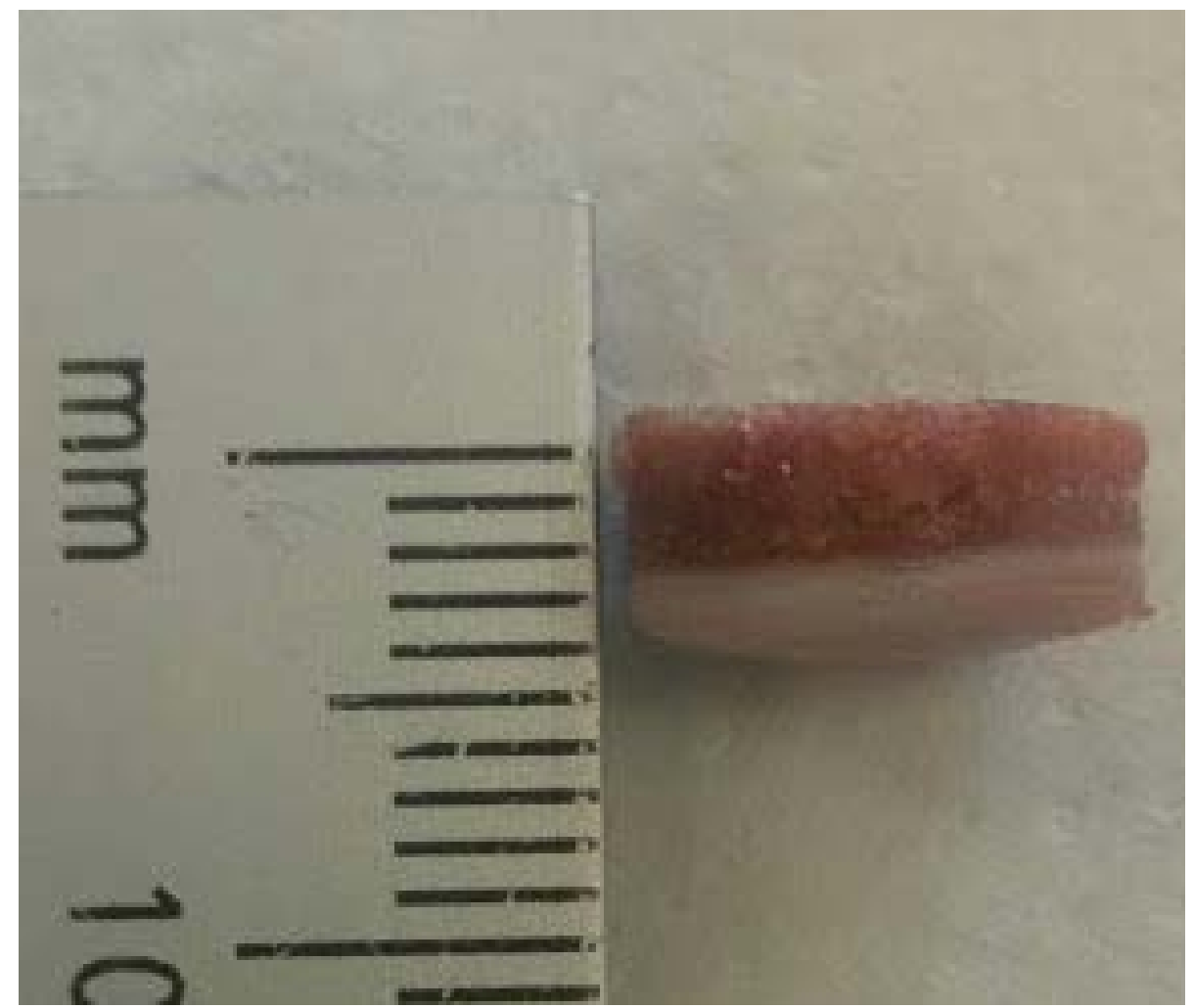

Illustration 5 Osteochondral porcine plug (diameter of $10 \mathrm{~mm}$, thickness of $4 \mathrm{~mm}$ )

Using a speed set epoxy, the plug was fixed into the center of a plastic washer. The bottom chamber of the diffusion chamber was filled with distilled water. Water was chosen as the solvent since the fluid phase of cartilage is mostly water. An O-ring was placed on top of the stopper (which indicates the division of the two chambers). The epoxy was allowed to set for 20 minutes before placing the washer with osteochondral plug on top of the O-ring. A marine grade silicone was used along the edge of the washer to seal the bottom chamber from the top chamber. The washer and its osteochondral plug then became the boundary between the two chambers, with the osteochondral plug acting as a 
membrane, being the only path for diffusion between the two chambers. Gadovist ${ }^{\circledR}$, a solution consisting of $1.0 \mathrm{mMol} / \mathrm{cm}^{3}$ of gadobutrol, was mixed with distilled water. Gadobutrol is a gadolinium-based contrast agent with a molecular weight of 605Da. The final concentration of gadobutrol diluted in distilled water was approximately 0.125 $\mathrm{mMol} / \mathrm{cm}^{3}$. This concentration was chosen to reflect the approximate concentration of gadobutrol in the blood stream when patients are imaged with contrast agents. The diffusion chamber was then closed using a plastic hose stopper.

\subsubsection{Imaging}

The diffusion chamber was secured on a bed intended for small animal imaging using Velcro strips as well as masking tape. The diffusion chamber was placed so that the normal of the sample's bone and cartilage surface were oriented near parallel to the static magnetic field $\left(\mathrm{B}_{0}\right)$. A standard radiofrequency $(\mathrm{RF})$ loop-shaped MRI coil, specially made for small animal imaging, was secured directly on top, as close as possible to the diffusion chamber and aligned with the osteochondral plug to ensure the best possible imaging field included the sample location. RF coils are frequently employed in various imaging protocols including small animal imaging to increase the signal-to-noise ratio [88]. The bed was then inserted into the MRI bore. For this experiment, a 7 Tesla pre-clinical MRI scanner was employed (Agilent/GE MR 901 system). After some preliminary imaging, it was determined that the $\mathrm{T}_{1}$-weighted fast spin echo imaging sequence provided images with the highest resolution. Each image represented a region of $35 \times 70 \mathrm{~mm}$ and contained $256 \times 512$ pixels, providing a resolution of $0.137 \mathrm{~mm}$ in each image dimension. Each pixel represented a 32-bit grey-scale value. Before beginning imaging with Gadovist ${ }^{\circledR}$, each 
sample was imaged without Gadovist ${ }^{\circledR}$ to have reference images. The MRI was pre-set to automatically image the sample every 5 minutes for 44 to 61 hours depending on the availability of the scanner, see Table 3 . The scan of the first sample experienced some challenges after the first three images were captured when the MRI software malfunctioned and no images were captured for 2 hours after the first three images. The scanner was restarted and continued imaging for the last 50 hours of a 52-hour exposure to gadobutrol. After samples 1, 2 and 3 it was unclear from if equilibrium was reached when imaging less than 50 hours and so the imaging time was increased for the remaining samples.

Table 3 MRI Imaging times for samples 1-7

\begin{tabular}{|c|c|}
\hline Sample number & $\begin{array}{c}\text { Imaging Time } \\
\text { (hours) }\end{array}$ \\
\hline $\mathbf{1}$ & $52^{*}$ \\
\hline $\mathbf{2}$ & 44.5 \\
\hline $\mathbf{3}$ & 46.2 \\
\hline $\mathbf{4}$ & 61 \\
\hline $\mathbf{5}$ & 61 \\
\hline $\mathbf{6}$ & 54.5 \\
\hline $\mathbf{7}$ & 61 \\
\hline
\end{tabular}

A phantom of water was added to the setup for samples 2 to 7 to aid in correcting drifting and minimize any post-imaging correction after the first sample was imaged with significant drifting. A phantom is a common method employed to correct drifting and reduce noise [89].

\subsubsection{Tracer Quantification}

Once the imaging sequence was completed, all images were opened in ImageJ, an image processing program, to produce image greyscale values of the bone, the cartilage and the distilled water below the cartilage. This was achieved using the region of interest 
(ROI) manager. Six to twelve ROIs were identified, depending on the cartilage thickness (see Illustration 6 and Table 4).

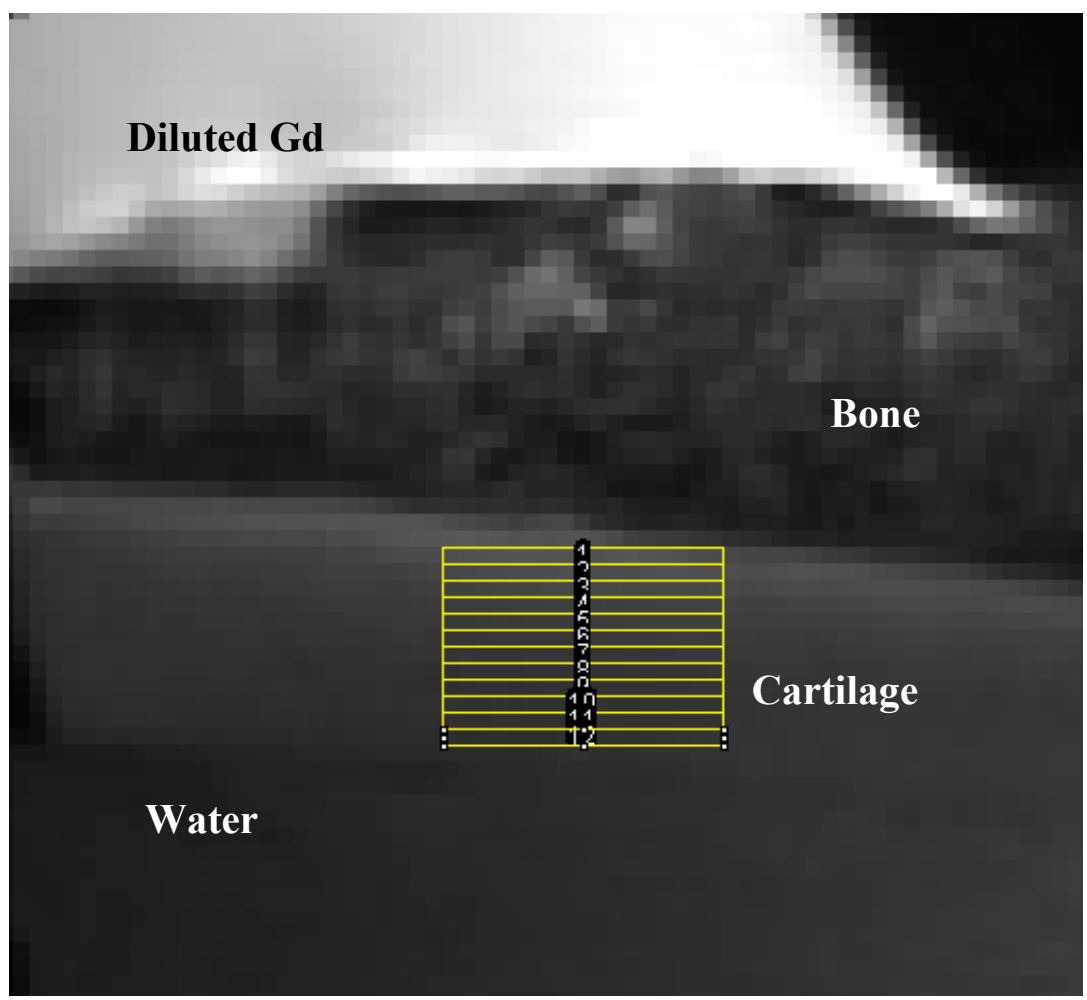

Illustration 6 MR image ROI selection for Sample 2

Table 4 Number of ROIs for each sample

\begin{tabular}{|c|c|}
\hline Sample number & Number of ROIs \\
\hline 1 & 6 \\
\hline 2 & 12 \\
\hline 3 & 8 \\
\hline 4 & 11 \\
5 & 6 \\
\hline 6 & 9 \\
\hline 7 & 11 \\
\hline
\end{tabular}


The middle $y$-value of the first ROI for each sample was selected at approximately $0.025 \mathrm{~cm}$ away from the middle of the tidemark. This distance was chosen because any distance closer was still considered to be part of the osteochondral junction image. Each ROI had an approximate height of $0.0135 \mathrm{~cm}$ with a width varying between 0.108 and $0.203 \mathrm{~cm}$. The software produced average image intensities for each ROI, at each time point (every 5 minutes). For analytical purposes, the intensity was observed at the following time points: 0 hours, 2 hours, 5 hours, 10 hours, 20 hours, 40 hours, 50 hours and 60 hours, when applicable. Each average intensity was converted to relative intensity by diving it by the initial intensity for the respective ROI (before gadobutrol was added):

$$
I_{t-r e l}=\frac{I_{t}}{I_{0}}
$$

It is assumed that the relative intensity is proportional to the concentration of the relative concentration of gadobutrol. The relationship between intensity and signal is as follows:

$$
\frac{S}{S_{0}}=\frac{I}{I_{0}}
$$

The relative intensities of each ROI for each image were converted to concentration values. This was achieved by first determining the apparent density of bone using the literary values of porcine bone density, $0.34 \mathrm{~g} / \mathrm{cm}^{3}$, and the following equation $[34,90]$ :

$$
\rho_{\text {apparent }}=\rho_{\text {tissue }}(1-\emptyset)
$$

In this equation, $\varnothing$ is the porosity of bone. The apparent density of bone and the density of cartilage were used to determine the $\mathrm{T}_{1}{ }^{+}$and $\mathrm{T}_{2}{ }^{+}$values (without gadobutrol) for both bone and cartilage from literature [84]. The relaxivity values, $r 1$ and $r 2$, for gadobutrol in cartilage and bone are found in Table 5 [85-87]. 
Table 5 Relaxivity values of cartilage and water

\begin{tabular}{|c|c|c|}
\hline & $\mathbf{r}_{1}(\mathbf{m M o l} \cdot \mathbf{s})^{-\mathbf{1}}$ & $\mathbf{r}_{2}(\mathbf{m M o l} \cdot \mathbf{s})^{-\mathbf{1}}$ \\
\hline Cartilage & 5.76 & 8.59 \\
\cline { 2 - 3 } Water (Bone) & 4.16 & 5.55 \\
\hline
\end{tabular}

Because the bone region consists of a large, open-pore structure, and would be filled with distilled water, it is assumed to be an infinite source, and so its relaxivity values are the same as that of water. The $T_{1}$ and $T_{2}$ values of bone, cartilage and water could then be determined using the following equations:

$$
\begin{aligned}
& T_{1}=1000 /\left(r_{1} \cdot[G d]+\left(1000 / T_{1}^{+}\right)\right. \\
& T_{2}=1000 /\left(r_{2} \cdot[G d]+\left(1000 / T_{2}{ }^{+}\right)\right.
\end{aligned}
$$

These relationships can also be shown graphically in Illustrations 7 and 8:

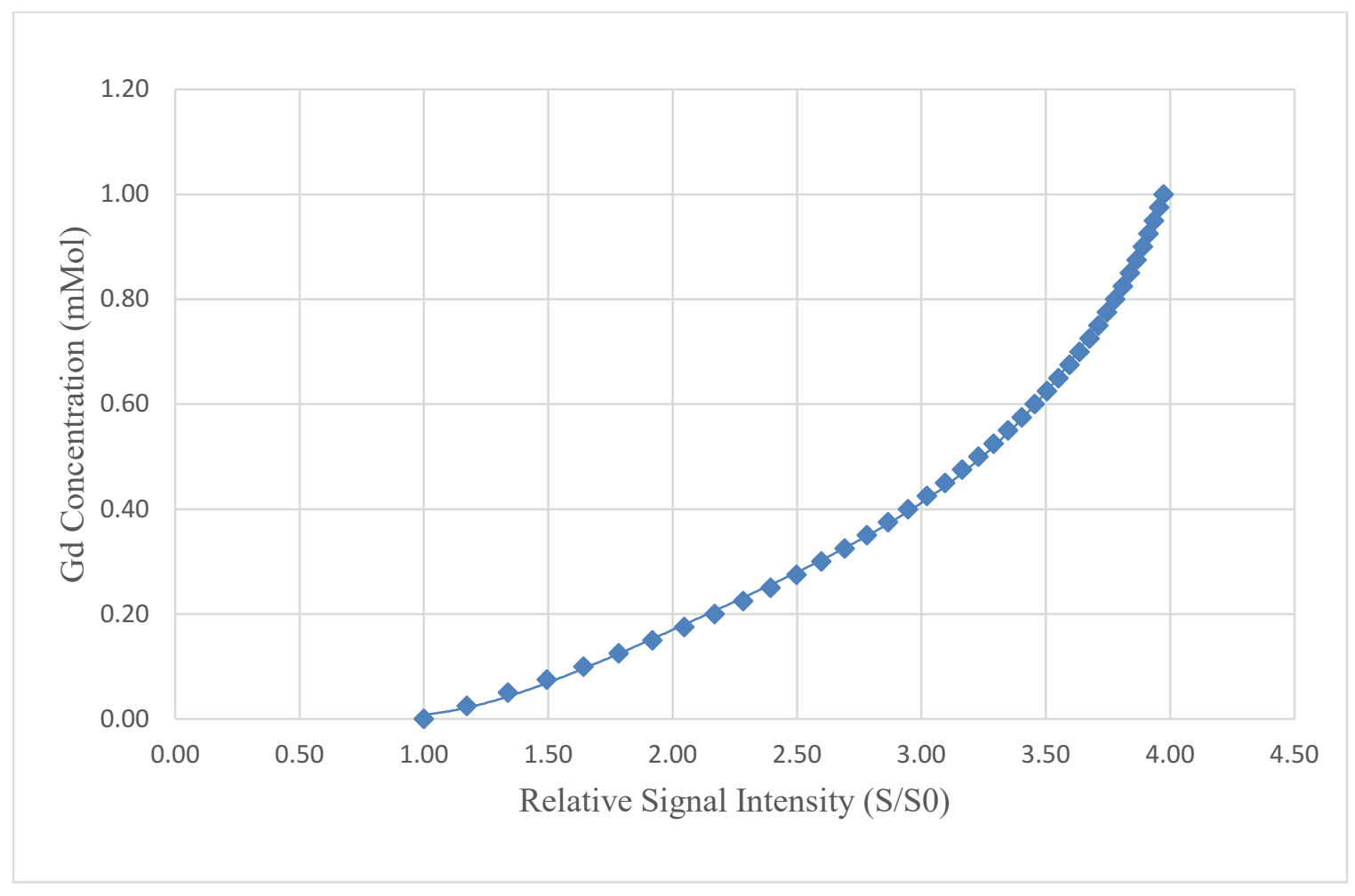

Illustration 7 Relationship between cartilage MR signal and gadobutrol concentration 


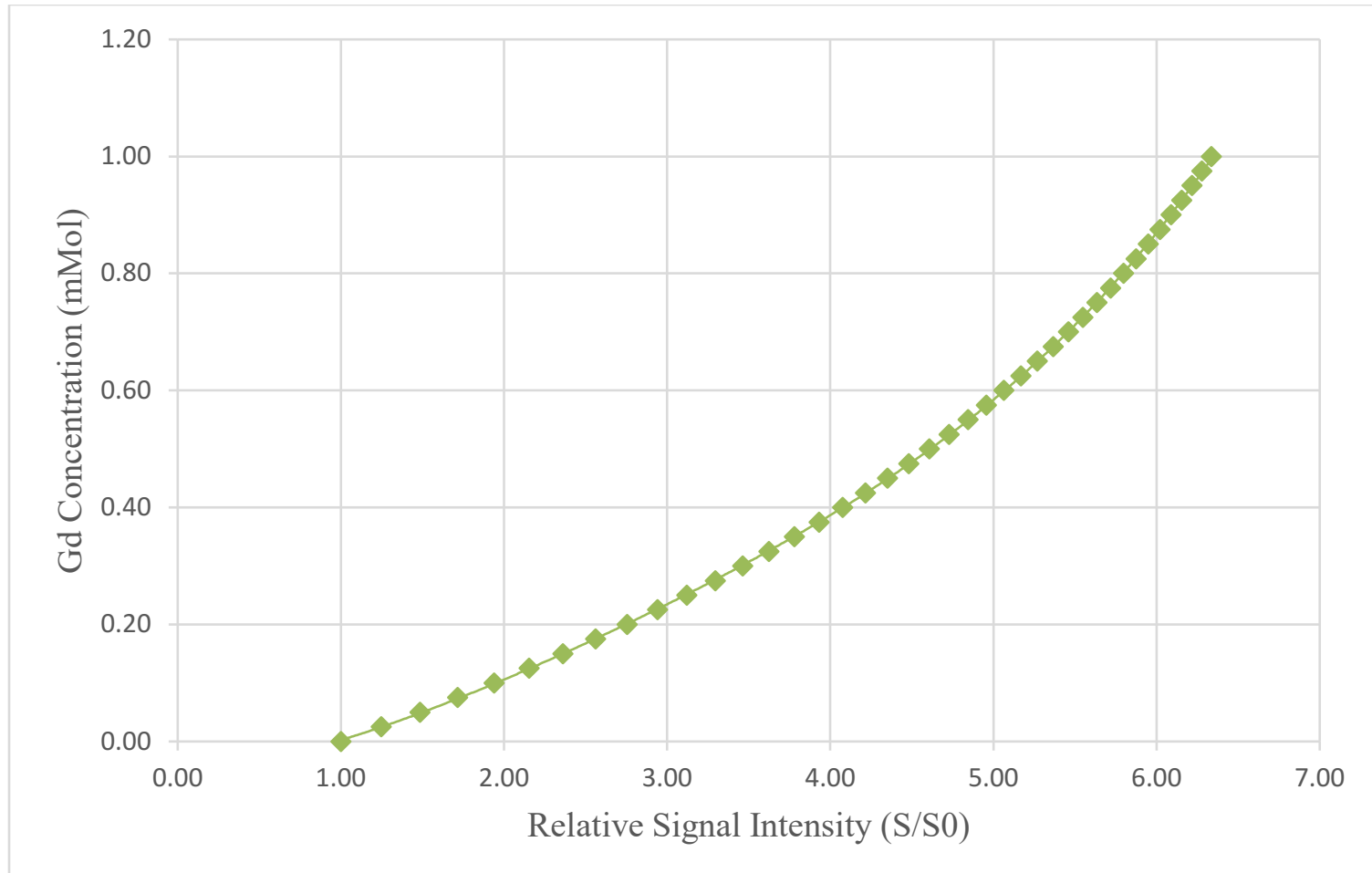

Illustration 8 Relationship between bone MR signal and gadobutrol concentration

The gadobutrol concentration [Gd] can then be found using the following equation [86]:

$$
[G d]=\frac{1}{r_{1}}\left(\frac{1}{T_{1}^{+}}-\frac{1}{T_{1}^{o}}\right)
$$

Where the $\mathrm{T}_{1}{ }^{+}$represents the $\mathrm{T}_{1}$ value for the tissue without contrast agent and $\mathrm{T}_{1}{ }^{0}$ represents the $T_{1}$ value with gadobutrol. The relationship between the signal and the $T 1$ and $\mathrm{T} 2$ values is defined as follows [86]:

$$
s=e^{-(12.3 / T 2)} \cdot\left(1-e^{\left(-\frac{300}{T 1}\right)}\right)
$$

Finally, the diffusivity at the selected time points could be determined using the onedimensional diffusion equation and the distribution of gadobutrol concentration $\mathrm{C}(\mathrm{x}, \mathrm{t})$ [26]:

$$
C(x, t)=C(x, \infty) \operatorname{erfc}(x / 2 \sqrt{D t})
$$


Where $\mathrm{x}$ is the distance from the osteochondral junction, $\mathrm{t}$ is the time, $\infty$ is taken as the last image acquired, $\mathrm{D}$ is the diffusivity and erfe is the complimentary error function. For this study, it was assumed that the bone was porous and provided no barrier to diffuse and could therefore be assumed as an infinite source. A purpose-written MATLAB ${ }^{\circledR}$ code and the curve fitting tool were employed to solve for diffusivity.

\subsection{Results}

The MR images obtained at time 0 hours, show very low signal intensity for both bone and cartilage (Illustration 9).

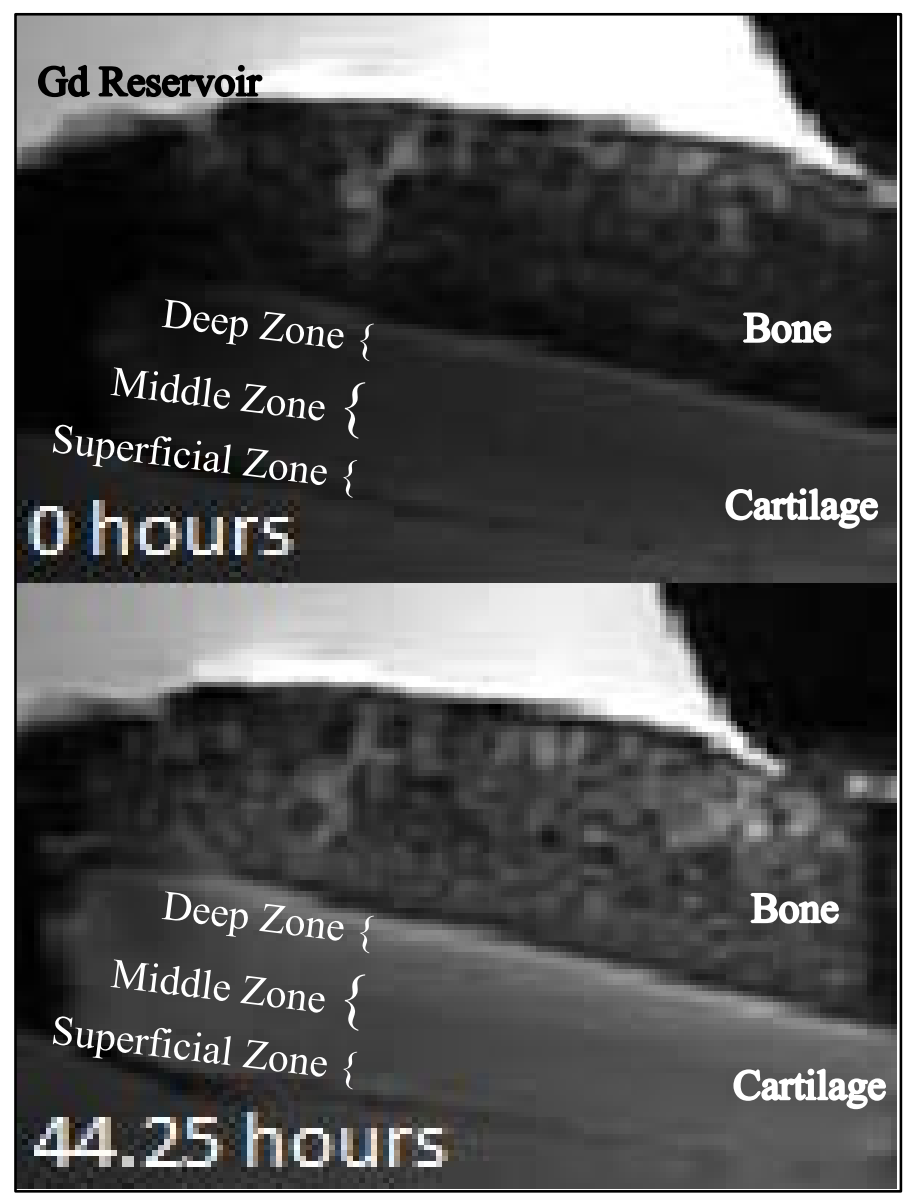

Illustration 9 Sample 2 MR image at 0 hours and 44.25 hours 
The distribution of gadobutrol was non-uniform, with the greatest uptake of tracer in the deep zone and the lesser uptake in the superficial zone (Illustration 10). The deep zone ( 0.027 to $0.041 \mathrm{~cm}$ depth) seemed to be nearing its point of equilibrium after 40 hours of exposure, while the middle and superficial zone concentrations continued to increase the entire 60 hours. The superficial zone in particular, had a lower concentration which is consistent with its high diffusivity [26].

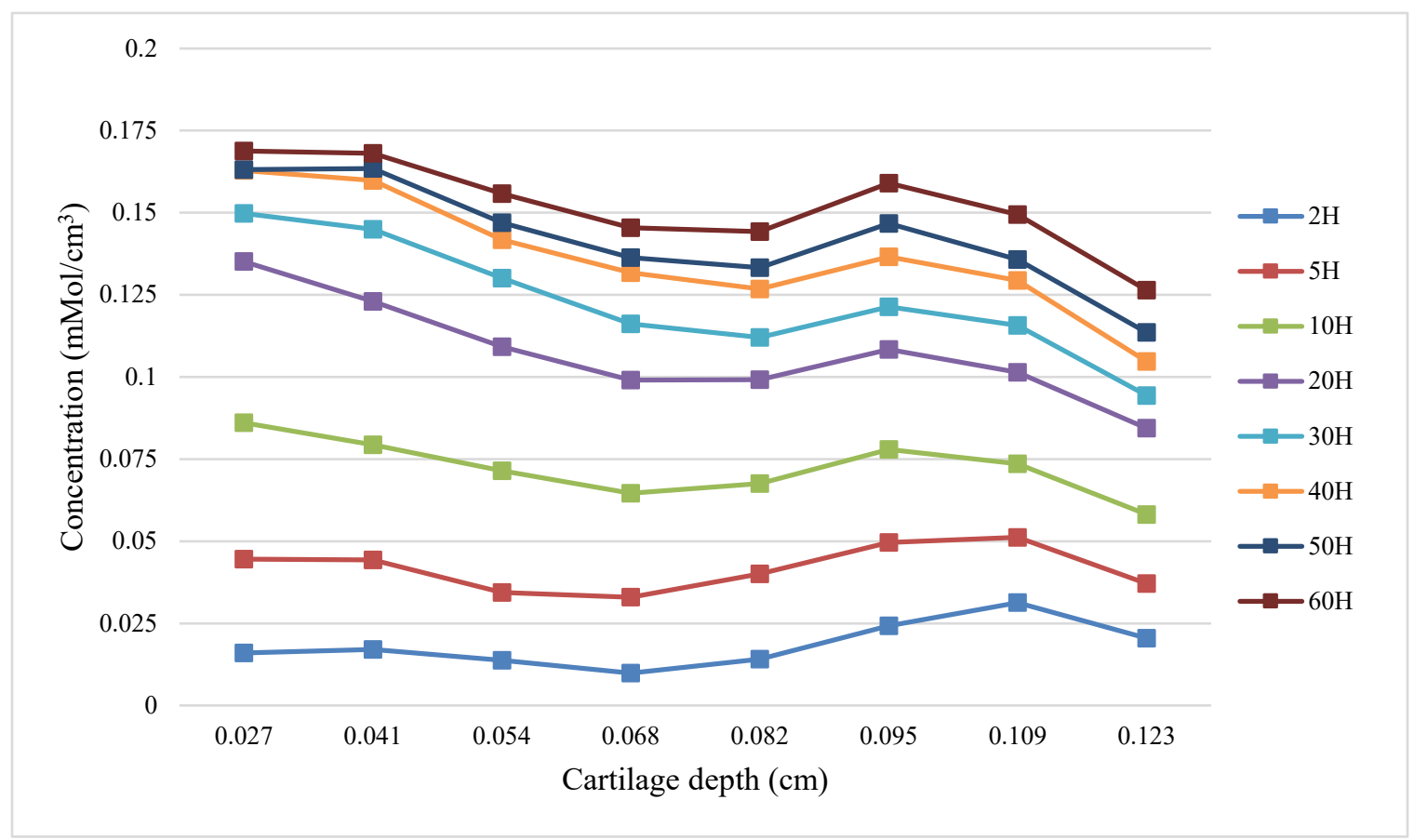

Illustration 10 Average Gd concentration at select times and cartilage depths for all samples

Concentration graphs for all samples individually can be found in Appendix B. 
Table 6 Diffusivity values of Samples 1-7 for select times

\begin{tabular}{|c|c|c|c|c|c|c|c|c|c|}
\hline \multirow[b]{3}{*}{ hours } & \multicolumn{9}{|c|}{ diffusivity $\left(\times 10^{-6} \mathrm{~cm}^{2 /} \mathrm{s}\right)$} \\
\hline & \multicolumn{7}{|c|}{ sample } & \multirow[b]{2}{*}{ mean } & \multirow{2}{*}{$\begin{array}{l}\text { standard } \\
\text { deviation }\end{array}$} \\
\hline & 1 & 2 & 3 & 4 & 5 & 6 & 7 & & \\
\hline 2 & 0.044 & 0.285 & 0.339 & 0.029 & 0.037 & 0.283 & 0.053 & 0.153 & 0.0171 \\
\hline 5 & 0.082 & 0.352 & 0.367 & 0.126 & 0.107 & 0.003 & 0.015 & 0.150 & 0.0192 \\
\hline 10 & 0.073 & 0.340 & 0.432 & 0.220 & 0.213 & 0.074 & 0.046 & 0.200 & 0.0185 \\
\hline 20 & 0.174 & 0.338 & 0.310 & 0.247 & 0.220 & 0.162 & 0.094 & 0.221 & 0.0063 \\
\hline 30 & 0.203 & 0.333 & 0.273 & 0.234 & 0.192 & 0.186 & 0.130 & 0.221 & 0.0037 \\
\hline 40 & & 0.277 & 0.196 & 0.215 & 0.186 & 0.215 & 0.163 & 0.209 & 0.0012 \\
\hline 50 & & & & 0.198 & 0.194 & 0.223 & 0.183 & 0.200 & 0.0002 \\
\hline
\end{tabular}

Table 7 Diffusivity values of Samples 1-7 by distance from the tidemark

\begin{tabular}{|c|c|c|c|c|c|c|c|c|c|}
\hline \multirow{3}{*}{$\begin{array}{l}\text { distance } \\
\text { from } \\
\text { tidemark } \\
\text { (cm) }\end{array}$} & \multicolumn{9}{|c|}{ diffusivity $\left(x 10^{-6} \mathrm{~cm}^{2} / \mathrm{s}\right)$} \\
\hline & \multicolumn{7}{|c|}{ sample } & \multirow[b]{2}{*}{ mean } & \multirow{2}{*}{$\begin{array}{l}\text { standard } \\
\text { deviation }\end{array}$} \\
\hline & 1 & 2 & 3 & 4 & 5 & 6 & 7 & & \\
\hline 0.027 & 0.030 & 0.443 & 0.665 & 0.031 & 0.049 & 0.049 & 0.022 & 0.184 & 0.058 \\
\hline 0.041 & 0.068 & 0.108 & 2.89 & 0.065 & 0.053 & 0.052 & 0.025 & 0.460 & 0.951 \\
\hline 0.054 & 0.141 & 0.167 & 21.5 & 0.100 & 0.083 & 0.079 & 0.048 & 3.160 & 56.06 \\
\hline 0.068 & 0.313 & 0.263 & 1.684 & 0.148 & 0.136 & 0.088 & 0.082 & 0.388 & 0.287 \\
\hline 0.082 & 0.376 & 0.351 & 1.183 & 0.231 & 0.200 & 0.123 & 0.114 & 0.368 & 0.120 \\
\hline 0.095 & & 0.515 & 1.039 & 0.337 & 0.252 & 0.156 & 0.133 & 0.405 & 0.096 \\
\hline 0.109 & & 0.651 & 0.867 & 0.489 & & 0.228 & 0.171 & 0.481 & 0.068 \\
\hline 0.123 & & 0.986 & 0.824 & 0.519 & & 0.331 & 0.200 & 0.572 & 0.087 \\
\hline 0.136 & & 1.06 & & 0.699 & & 0.416 & 0.237 & 0.602 & 0.096 \\
\hline 0.15 & & 1.169 & & 0.649 & & & 0.267 & 0.695 & 0.137 \\
\hline 0.164 & & 1.360 & & 0.673 & & & 0.332 & 0.788 & 0.183 \\
\hline
\end{tabular}




\begin{tabular}{|l|l|l|l|l|l|l|l|}
\hline \multirow{2}{*}{$\begin{array}{l}\text { distance } \\
\text { from }\end{array}$} & \multicolumn{5}{|l|}{ Diffusivity $\left(\mathrm{x} 10-6 \mathrm{~cm}^{2} / \mathrm{s}\right)$} \\
\cline { 2 - 8 } & Sample & Sample & Sample & Sample & \multicolumn{2}{l|}{ Sample } & \multicolumn{2}{l|}{ Sample } & Sample \\
tidemark & 1 & 2 & 3 & 4 & 5 & 6 & 7 \\
\hline 0.027 & 0.030 & 0.443 & 0.665 & 0.031 & 0.049 & 0.049 & 0.022 \\
\hline 0.041 & 0.068 & 0.108 & 2.848 & 0.065 & 0.053 & 0.052 & 0.025 \\
\hline 0.054 & 0.141 & 0.167 & 21.500 & 0.100 & 0.083 & 0.079 & 0.048 \\
\hline 0.068 & 0.313 & 0.263 & 1.684 & 0.148 & 0.136 & 0.088 & 0.082 \\
\hline 0.082 & 0.376 & 0.351 & 1.183 & 0.231 & 0.200 & 0.123 & 0.114 \\
\hline 0.095 & & 0.515 & 1.039 & 0.337 & 0.252 & 0.156 & 0.133 \\
\hline 0.109 & & 0.651 & 0.867 & 0.489 & & 0.228 & 0.171 \\
\hline 0.123 & & 0.986 & 0.824 & 0.519 & & 0.331 & 0.200 \\
\hline 0.136 & & 1.055 & & 0.699 & & 0.416 & 0.237 \\
\hline 0.15 & & 1.169 & & 0.649 & & & 0.267 \\
\hline 0.164 & & 1.360 & & 0.673 & & & 0.332 \\
\hline
\end{tabular}

The average diffusivity of all samples, for the entire cartilage, at each selected time point can be seen in Illustration 11. The diffusivity values for select time and for select locations can be seen in Tables 6 and 7, respectively. Looking at Illustration 11 it appears diffusivity values are converging over time. The average diffusivity values for the duration of the experiment, by distance from the tidemark can be seen in Illustration 12 . 


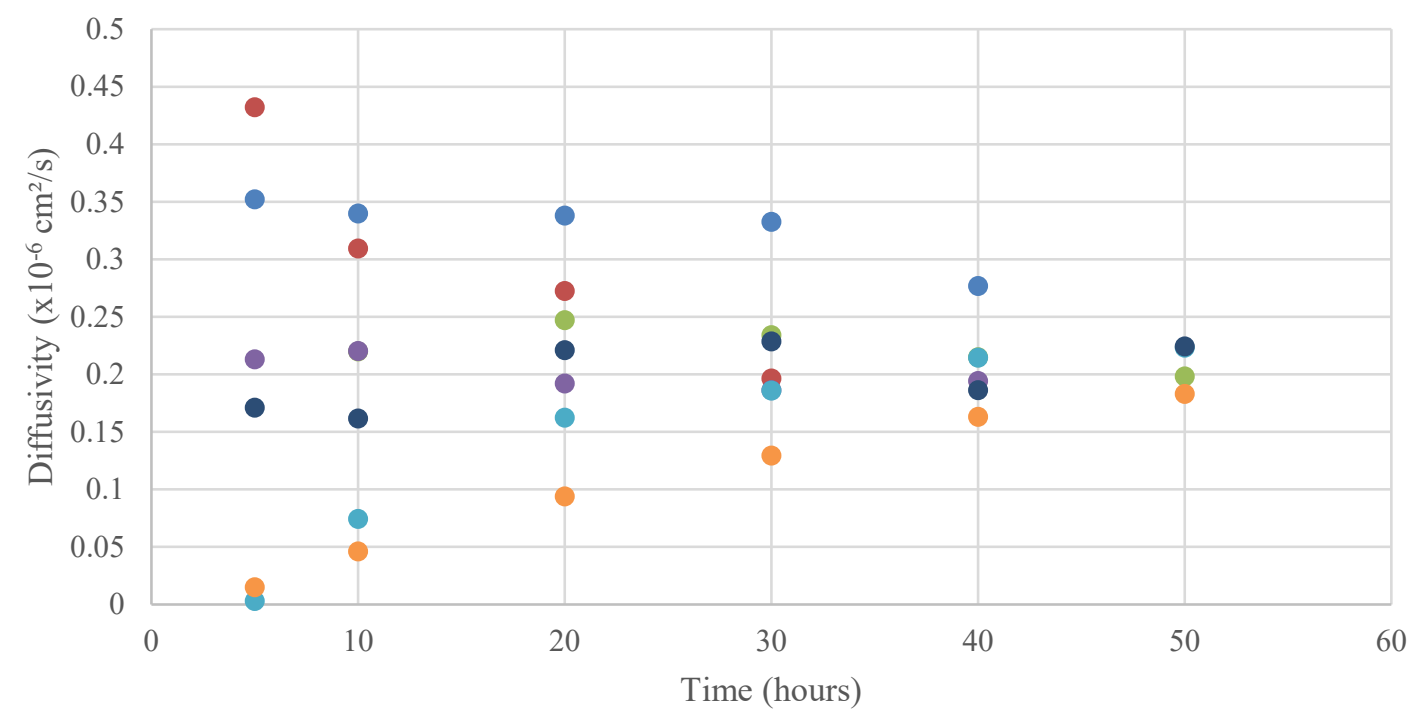

Illustration 11 Diffusivity of Gd through cartilage for durations up to 50 hours

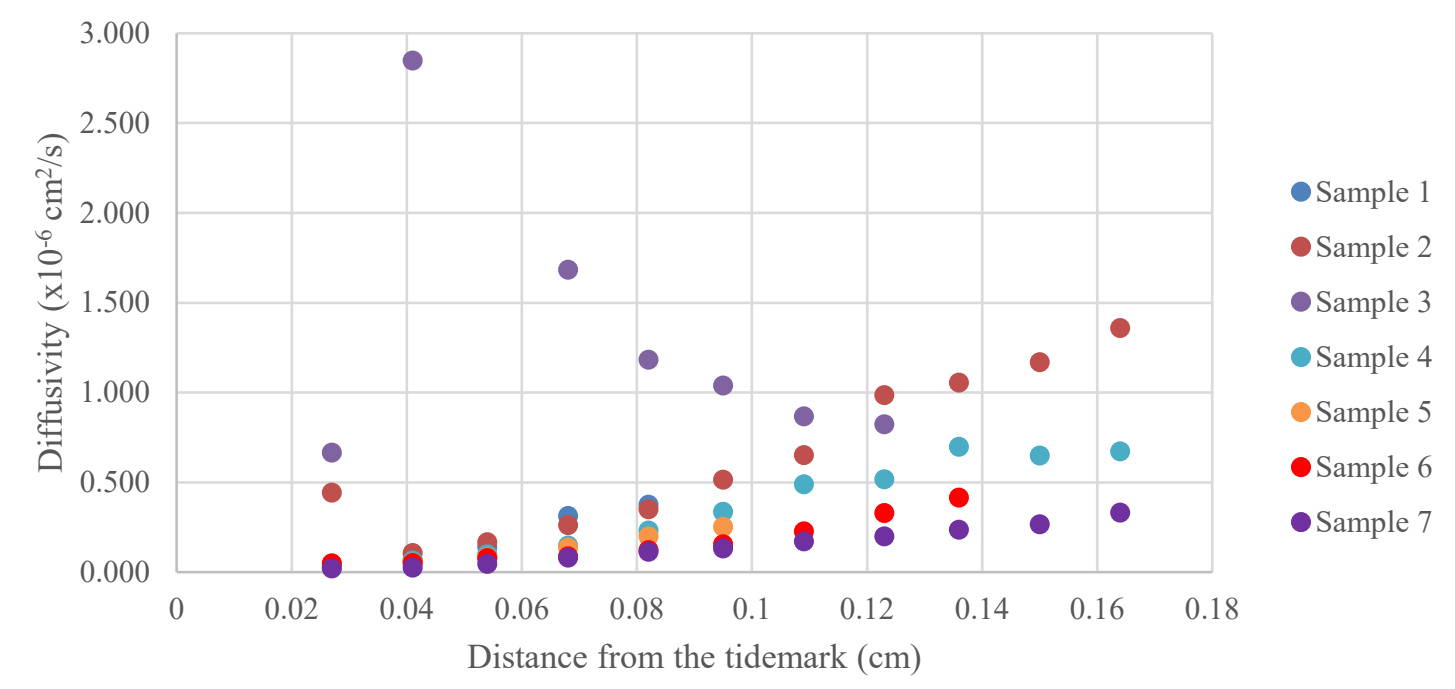

Illustration 12 Diffusivity values of Gd through cartilage at select distances from the tidemark 


\subsection{Discussion}

The aim of this experiment was to measure the diffusion of Gadovist ${ }^{\circledR}$ throughout the cartilage depth. Looking at the first and last images it was immediately apparent that the image intensity had increased in both the bone and cartilage.

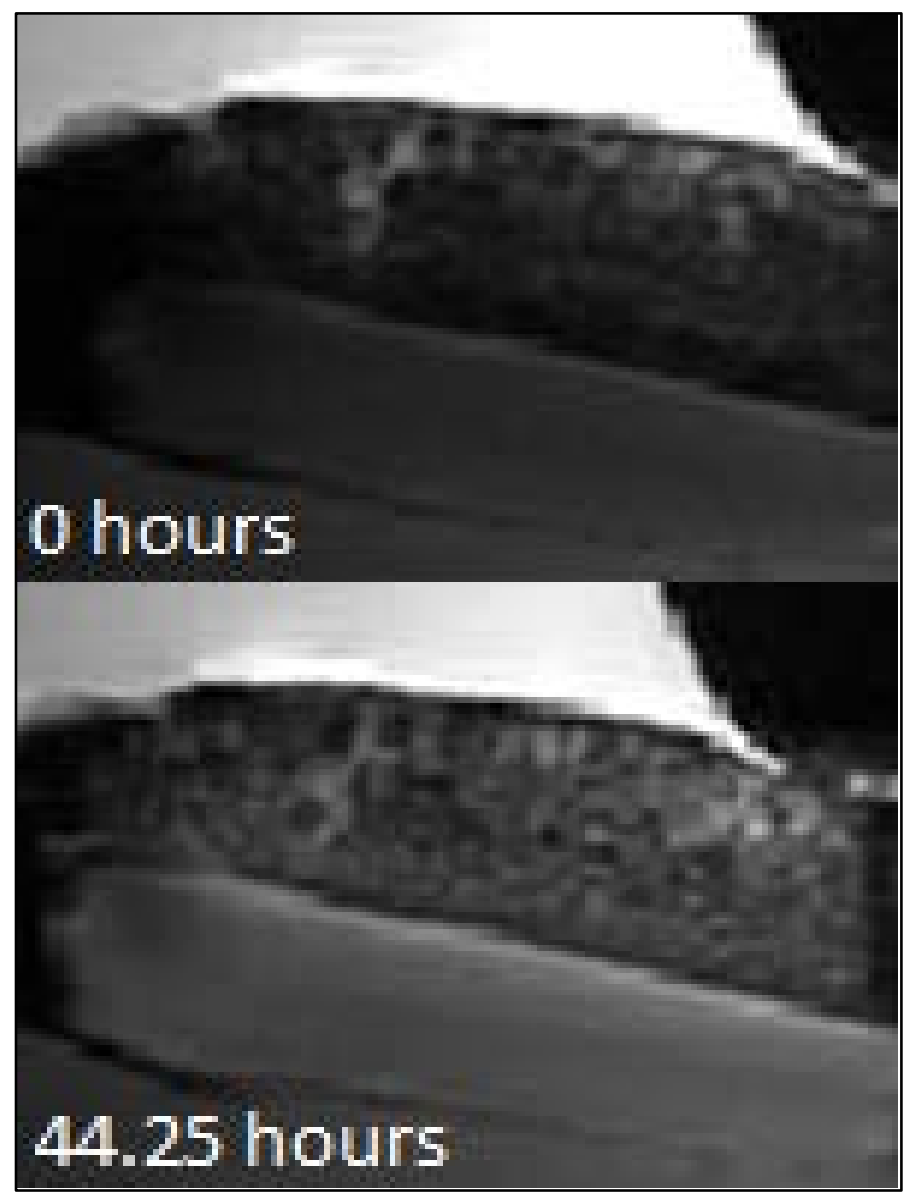

Illustration 13 Comparison of first and last images of sample 2

The image above clearly demonstrates an uptake of Gadovist ${ }^{\circledR}$ throughout the cartilage depth. Diffusivity of the contrast agent through the cartilage was successfully measured.

As can be seen in Illustration 13, the bone typically images as black (low signal) since the relaxation time of bone is too fast for standard MR imaging. Although the contrast 
agent is seen in the bone after 44.25 hours, it can be seen that a thin layer directly above the cartilage remains black. This is a thin layer of subchondral bone which may act as a barrier to diffusion.

MRI was chosen as the imaging modality because it permits for continuous, nondestructive imaging for long periods of time. Gadovist ${ }^{\circledR}$ was an excellent option because of its low molecular weight, neutral charge and high imaging intensity. It is also approved by Health Canada and the Food and Drug Administration for use in humans and thus could be used in future studies in patients. The images acquired were of good quality for the scale of the sample. The resolution allowed for precise monitoring of gadobutrol migration. Motion artifact was detected in one of the images at a specific time for one specimen, however the diffusivity data was consistent throughout the samples. It appears in Illustration 10 that the concentration in the deep zone locations (select points closest to the tidemark) seems to be nearing equilibrium as the rate of increase of concentration is decreasing. The results clearly demonstrated that the bone-cartilage boundary was permeable to the contrast agent and it diffused throughout the cartilage layers. Illustration 12 demonstrates the increase in diffusivity values with increasing distance from the tidemark which further confirms that the tidemark hinders diffusion, but once past this barrier the diffusivity increases. Further study is required to assess the relationship between regional variation of diffusivity with variation in proteoglycan content and collagen orientation. However the concentration of gadobutrol was consistent with the known orientation of collagen fibers and the water content in each cartilage zone [95]. In the selected locations, furthest from the tidemark (superficial zone), it appears that the rate at 
which the concentration is increasing is not lowering like that of the deep zone and so this zone is not nearing equilibrium.

It is likely that the gadobutrol had some chemical interactions with the extracellular matrix of cartilage which would influence its rate of diffusion and its concentration. The deep zone demonstrated highest uptake of tracer and the superficial zone demonstrating the lowest which is consistent with the proteoglycan distribution. This also agrees with studies that showed anisotropic permeability to water flow in poroelastic cartilage models that varies with collagen orientation throughout the tissue [95]. If OA leads to a decreased amount of proteoglycans, tracer uptake would increase. The implications of these results are not only for research purposes but could also provide evidence of the use of contrastenhanced MRIs in clinical settings for diagnosis and monitoring. The uptake of tracer could possibly be used as a measure of OA progression, but requires further investigation using tissue samples with varying degeneration. The current results also confirm the theory that a plausible mechanism for cartilage to receive its nutrients and dispose of metabolites is through diffusion across osteochondral junction. These results also agree with a similar study conducted by Arkill where fluorescent dyes of similar molecular weight were employed instead of contrast agents, although tests were destructive [26].

The diffusivity values showed variability at short exposure times, however they were more consistent at longer exposure times. Although diffusivity was calculated for various exposure times, in each sample, it is expected to be a constant material parameter for each specimen. The observed variability could be due to the subchondral bone resistivity affecting short-term measurements of concentration. At longer times the distribution of gadobutrol is determined by the diffusivity of the cartilage rather than the 
barrier of the subchondral bone. For practical significance, it's recommended to run the experiment for a minimum of 50 hours to calculate the diffusivity value to ensure the resistivity is not affecting the diffusivity. The influence of the tidemark to gadobutrol diffusion will be investigated in the next chapter.

A sensitivity analysis on the bit-depth of the MRI images was undertaken by changing the bit size from its original 32-bit to 16-bit for sample 2, by post-imaging artificial bit reduction using ImageJ. Qualitatively, when switching from 32-bit to 16-bit the 16-bit image was visibly brighter (Illustrations 14 and 15).

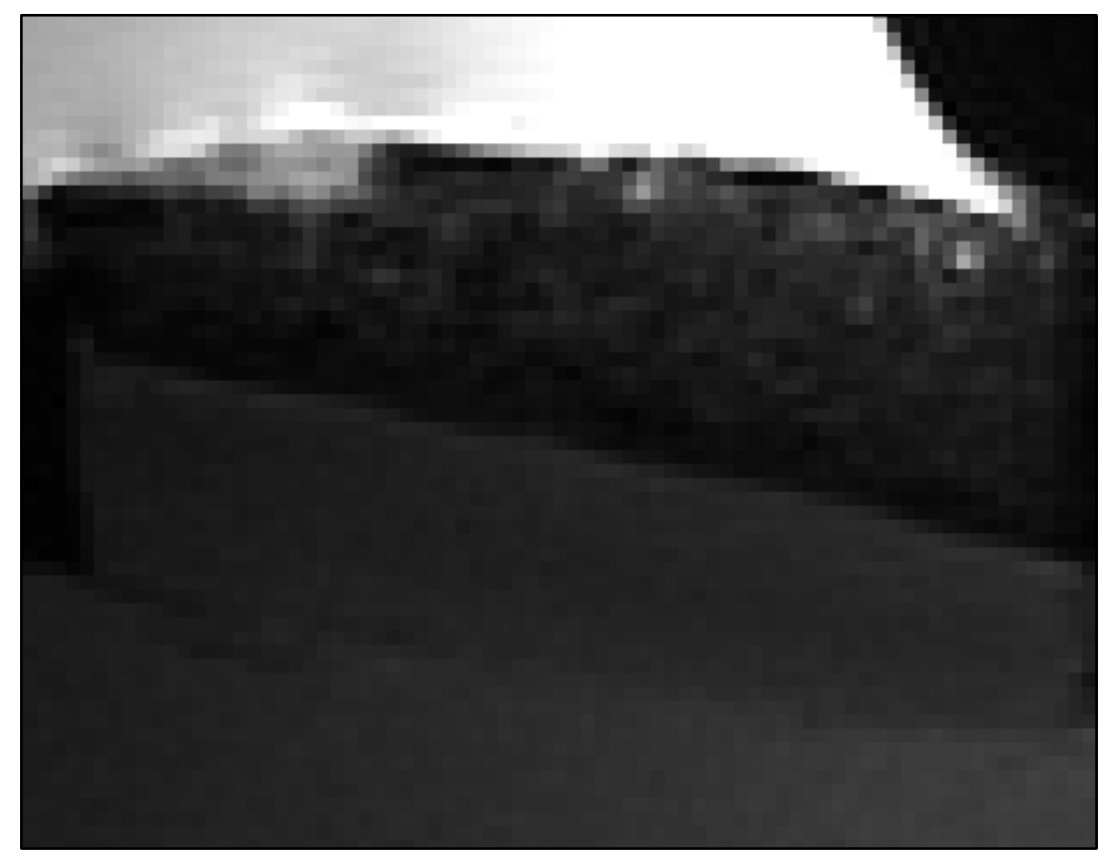

Illustration 14 32-bit image of Sample 2 at 0 hours

Quantitatively, the concentration values increased anywhere between 0.1 and 0.04 $\mathrm{mMol} / \mathrm{cm}^{3}$, with a root mean square error value of $0.041 \mathrm{mMol} / \mathrm{cm}^{3}$ and a mean absolute error value of $0.031 \mathrm{mMol} / \mathrm{cm}^{3}$. The average diffusivity value of sample 2 increased from $0.0703 \mathrm{~cm}^{2} / \mathrm{s}$ to $0.1077 \mathrm{~cm}^{2} / \mathrm{s}$. Another sensitivity analysis was conducted by changing the 


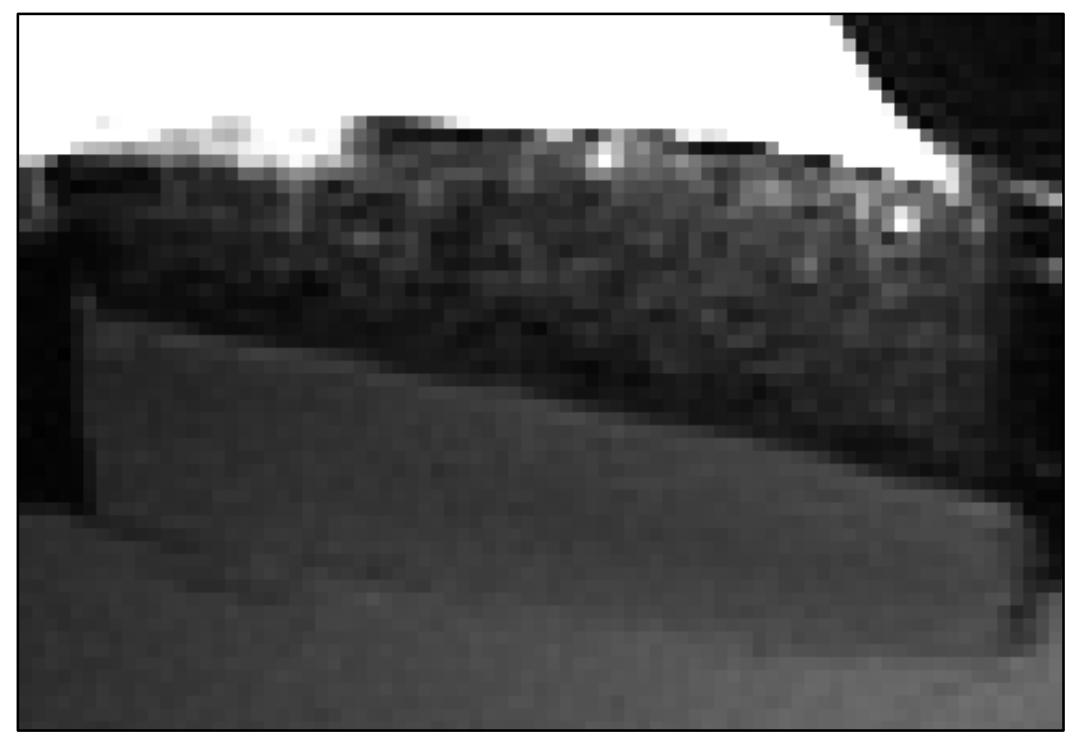

Illustration 15 16-bit image of sample 2 at 0 hours

relaxivity value $\mathrm{r}_{1}$ from $5.79 \mathrm{mMol}^{-1} \mathrm{~s}^{-1}$ to $4.79 \mathrm{mMol}^{-1} \mathrm{~s}^{-1}$ and $6.79 \mathrm{mMol}^{-1} \mathrm{~s}^{-1}$. In the first case, where relaxivity was decreased by $1.0 \mathrm{mMol}^{-1} \mathrm{~s}^{-1}$ the diffusivity was seen to increase from $0.0703 \mathrm{~cm}^{2} / \mathrm{s}$ to $0.1373 \mathrm{~cm}^{2} / \mathrm{s}$. In the second case where the relaxivity was increased by $1.0 \mathrm{mMol}^{-1} \mathrm{~s}^{-1}$ the diffusivity decreased to $0.0636 \mathrm{~cm}^{2} / \mathrm{s}$. Altering $\mathrm{r}_{1}$ value led to an opposite change in the diffusivity, i.e. increasing $r_{1}$ led to a decrease in diffusivity. Similarly, $\mathrm{r}_{2}$ was decreased by $1.0 \mathrm{mMol}^{-1} \mathrm{~s}^{-1}$ and the diffusivity was seen to increase to $0.1252 \mathrm{~cm}^{2} / \mathrm{s}$, while increasing by $1.0 \mathrm{mMol}^{-1} \mathrm{~s}^{-1}$ increased the diffusivity to $0.0373 \mathrm{~cm}^{2} / \mathrm{s}$. In conclusion, the diffusivity values determined are sensitive to the assumed relaxivity value for collagen: an increase in relaxivity, either $\mathrm{r} 1$ or $\mathrm{r} 2$ led to a decrease in diffusivity and a decreased in the bit-resolution led to an increase in diffusivity. Future work should be undertaken to experimentally determine the relaxivity values of pig bone and cartilage.

The temperature inside of the MRI scanner is expected to have increased to temperatures up to $35^{\circ} \mathrm{C}$. This inconsistency in temperature was not accounted for and is expected to have had an increase on the diffusion rate of the gadobutrol. It is unknown when the temperature would have reached its equilibrium point. 
To verify the silicone seal was not leaking or that the Gadovist ${ }^{\circledR}$ was not affecting the silicone a test was done with an impermeable plastic disk replacing the washer and osteochondral plug. The disk was sealed using the same marine grade silicone and imaged for 65 hours (Illustrations 16 and 17). If the Gadovist ${ }^{\circledR}$ were to leak through the silicone the intensity of the image of the water in the bottom chamber would increase over time. There was no change in intensity seen over the 65 hours ensuring that the only possible path of diffusion into the cartilage was through the bone (Illustration 18).

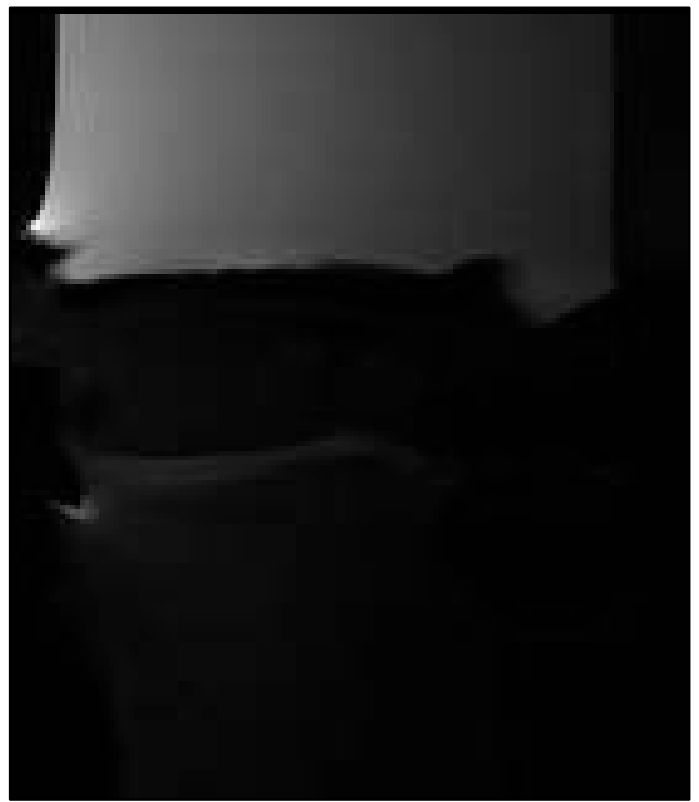

Illustration 16 Plastic disk MR image at 65 hours 


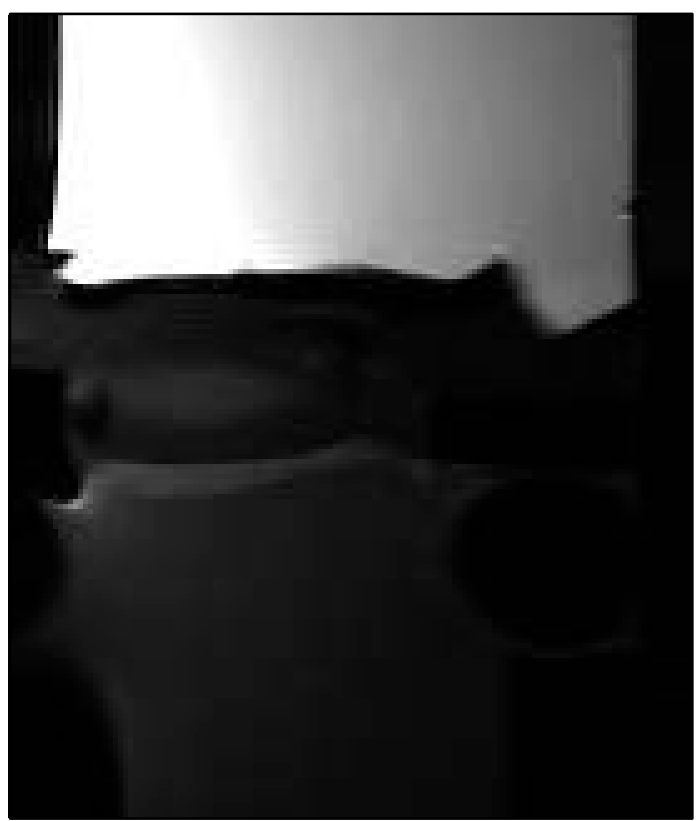

Illustration 17 Plastic disk MR image at 0 hours

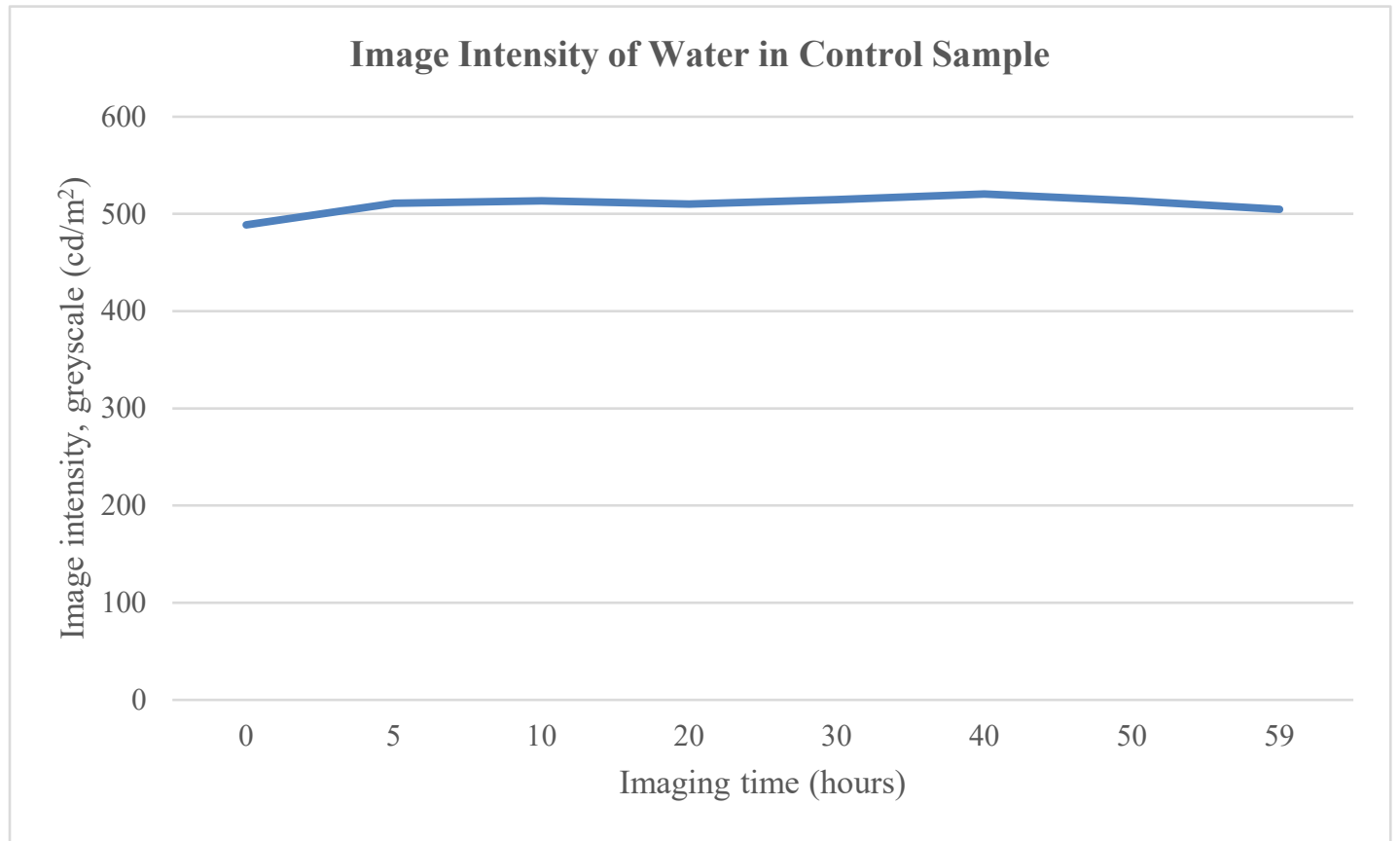

\section{Illustration 18 Image intensity of control sample}

Various limitations can be found with this study. Although pig bones are often thought to have similar properties to human bones, there is no way of knowing if porcine cartilage has different diffusion properties than human cartilage. The small sample size and relatively large standard deviations, especially for lower exposure times may render this 
impractical for clinical diagnosis but remains useful as a research tool. Although a control test was conducted, it is impossible to know if any one of the tests had a defective seal. As it can be seen in Illustration 10, Gd concentration in the superficial zone continues to increase and seems to have not yet reached equilibrium and so it is suggested that studies surpassing 60 hours of Gd exposure and imaging be undertaken to determine the time at which equilibrium is reached. Future work would also include investigating a change in diffusivity with joint degeneration. Equilibrium distribution of Gd is dependent on collagen orientation as well as proteoglycan concentration. If the diffusion of Gd is affected by joint degeneration, this method could be useful in assessing the progression of OA. Furthermore, the technique may be useful in assessing non-uniform changes in diffusivity through the tissue during degeneration. Distilled water was chosen as the solvent, however it does not perfectly mimic synovial fluid. The use of distilled water was consistent throughout samples and the goal of this study was to demonstrate that molecular diffusion could be measured with contrast-enhanced MRI and it is not expected that a different solvent would change this conclusion.

In conclusion, the passive molecular diffusion across the osteochondral junction can be measured using contrast-enhanced MR imaging. The distribution of gadobutrol was not consistent throughout the cartilage but rather demonstrated that gadobutrol concentration correlated to proteoglycan content with the deep zone showing the highest concentration of both proteoglycan and gadobutrol. This study showed that measurement of diffusion in osteochondral tissues can be performed using contrast-enhanced MRI which allows in vivo investigation of diffusion processes that may provide information about degenerative changes in diseases such as OA. 


\section{Chapter: Numerical simulation of diffusion in osteochondral samples}

\subsection{Introduction}

The focus of osteoarthritis research has shifted to consider the joint as a whole and more specifically the subchondral bone and its possible effects on the health of cartilage. $\mathrm{OA}$ is a disease that changes the composition and consequentially the structure of cartilage and subchondral bone. In cartilage, proteoglycan loss and consequentially alterations in water content are indications of OA and have the potential to be used as a way of measuring the progression of OA $[65,66]$. It has also been determined that bone undergoes structural changes in OA affected joints, such as increase in bone thickness, reduction of trabecular bone density and the development of bone marrow lesions [96]. Together, these changes affect the functionality and structure of the joint as a whole. What remains unknown is whether these changes affect the ability of solutes to diffuse across the osteochondral junction. As was shown in Chapter 2, nutrient-size solutes can diffuse across the osteochondral junction in healthy joints. Gaining a better understanding of the diffusivity threshold of external solutes across the osteochondral junction will help to better understand the structural differences between healthy and OA osteochondral components. Due to the destructive nature of the experiments undertaken in Chapter 2, it would be impossible to reproduce this experiment at various levels of OA on the same joint. For this purpose, it would be beneficial to simulate the Gd diffusion experiment and modify certain properties of the joint and observe the effects on Gd concentration distribution. We hypothesize that the osteochondral junction represents a barrier to molecule diffusion that may be measured by a resistivity. The resistivity of the osteochondral junction may be a 
function of the joint health and is expected to change as the structure of the bone and cartilage change during the progression of OA.

\subsubsection{Diffusion Theory}

Reproduction of the diffusion of Gd across the osteochondral junction requires the application of some diffusion laws. To measure the diffusion of a substance through another material, Fick's Laws of diffusion can be employed. Fick's first law is the following [83]:

$$
\Gamma=-D \frac{\partial C}{\partial x}
$$

In this equation $\Gamma$ represents the molar flux, a dimension that measures the amount of substance flowing per unit time $\left(\mathrm{mol} / \mathrm{m}^{2} \mathrm{~s}^{-1}\right)$. The concentration is represented by $\mathrm{C}$ and gives dimension to the amount of substance per unit volume $\left(\mathrm{mol} / \mathrm{m}^{3}\right)$. The diffusion coefficient or diffusivity (D), is a proportionality constant between the molar flux occurring due to diffusion and the concentration gradient of the material through which a substance is diffusing [83]. In this study the spatial unit (x) represents the depth of the material through which diffusion is occurring. Fick's first law only applies in steady state conditions. When concentration is a function of time, this becomes a partial differential equation, governed by Fick's Second Law [83]:

$$
\frac{\partial C}{\partial t}=D \frac{\partial^{2} C}{\partial x^{2}}
$$

The Gd must first diffuse through a thin layer of dense subchondral bone that acts as a barrier to diffusion. As shown in Chapter 2, the bottom image of Illustration 13, at 44 hours, a part of the bone directly above the tidemark remains black. This black 'line' is the thin 
layer of subchondral bone. We assume that the flux through the bone layer is proportional to the concentration difference across the layer:

$$
\left.\frac{d C}{d t}\right|_{x=0}=\frac{1}{R}\left(C_{b}-C(0, t)\right)
$$

Where $0 \leq \mathrm{x} \leq \mathrm{L}$ is the cartilage region. In this experiment bone is acting as a barrier to the diffusion of $\mathrm{Gd}$. The resistivity of the bone can be represented by $\mathrm{R}$ and $\mathrm{C}_{\mathrm{b}}$ is the constant solute concentration in the reservoir in contact with the bone (Illustration 19).

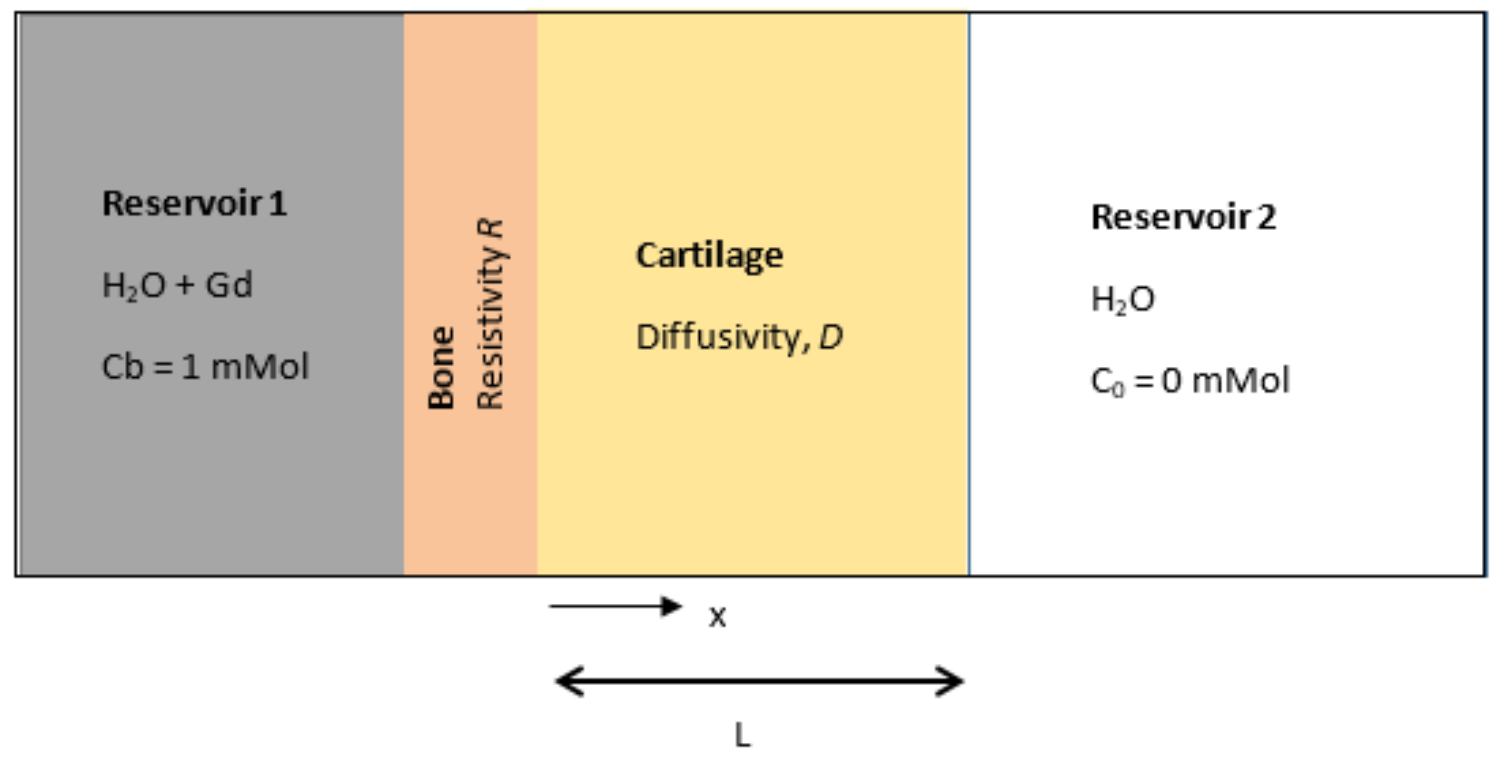

Illustration 19 Model of diffusion setup for FD analysis and simulation

To solve the diffusion equation for the cartilage some additional theory is required. The Crank-Nicolson method is a finite difference method that can be applied to solve partial differential equations such as Fick's Second law. The Crank-Nicolson method is often applied to solve linear 1D diffusion problems [101]. Applying the Crank-Nicolson method, the diffusion equation is given as:

$$
\frac{\partial C}{\partial t}=D \frac{\partial^{2} C}{\partial x^{2}}-U_{x} \frac{\partial C}{\partial x}
$$


Where $U_{x}$ is the flow velocity and $U_{x} \frac{\partial C}{\partial x}$ represents advection. With the Crank-Nicolson method, each component of the derivative is numerically approximated:

$$
\begin{aligned}
& \frac{\partial C}{\partial t} \rightarrow \frac{C_{i}^{j+1}-C_{i}^{j}}{\Delta t} \\
& \frac{\partial^{2} C}{\partial x^{2}} \rightarrow \frac{1}{2(\Delta x)^{2}}\left(\left(C_{i+1}^{j+1}-2 C_{i}^{j+1}+C_{i-1}^{j+1}\right)+\left(C_{i+1}^{j}-2 C_{i}^{j}+C_{i-1}^{j}\right)\right) \\
& \frac{\partial C}{\partial t} \rightarrow \frac{1}{2}\left(\frac{\left(C_{i+1}^{j+1}-C_{i-1}^{j+1}\right)}{2(\Delta x)}+\frac{\left(C_{i+1}^{j}-C_{i-1}^{j}\right)}{2(\Delta x)}\right) \\
& C \rightarrow \frac{1}{2}\left(C_{i}^{j+1}+C_{i}^{j}\right)
\end{aligned}
$$

To simplify, the following constants are created:

$$
\begin{aligned}
& \lambda=\frac{D_{x} \Delta t}{2 \Delta x^{2}} \\
& \alpha=\frac{U_{x} \Delta t}{4 \Delta x}
\end{aligned}
$$

In this study, there was no flow $\left(U_{x}=0\right)$ so constant $\alpha$ is 0 . Substituting equations 5-9 into equation 4 gives the following:

$$
\lambda C_{i-1}^{j+1}+2 \lambda C_{i}^{j+1}-\lambda C_{i+1}^{j+1}=\lambda C_{i-1}^{j}-2 \lambda C_{i}^{j}+\lambda C_{i+1}^{j}
$$

This equation can be solved in the matrix form:

$$
[A A]\left[C^{j+1}\right]=[B B]\left[C^{j}\right]+[d]
$$

To find the concentration at any time, the matrix equation can be rearranged as follows:

$$
\left[C^{j+1}\right]=\left[A A^{-1}\right]\left([B B]\left[C^{j}\right]+[d]\right)
$$

These matrices can become quite large depending on the number of nodes selected and thus it is recommended to solve using a numerical computing software such as MATLAB®. 


\subsection{Methods}

Diffusion of gadobutrol molecules in cartilage was modeled computationally by performing a one-dimensional (1D) finite difference (FD) analysis using the CrankNicolson method described above. This was implemented using MATLAB ${ }^{\circledR}$ software. The diffusion of gadobutrol was assumed to occur only in the thickness direction which allowed for the 1D model. The cartilage was taken to be a single zone, with constant properties throughout the cartilage. It was assumed that the sample was uniform both radially and across its thickness. The cartilage FD model used a 10-node, evenly distributed, linear element for mesh and the total length was specified to each of the experimental specimens being reproduced. Some simplifying boundary conditions could be applied. As was done in the experimental design, the diffusion was allowed only from the bone side of the specimen. The diffusivity values were assumed to be constant throughout the cartilage depth. The concentration of gadobutrol was assumed to remain constant at $1.0 \mathrm{mMol}$ in the upper chamber (left side of Illustration 19) and $0 \mathrm{mMol}$ in the lower water-filled chamber (right side of Illustration 19). The boundary and initial conditions for concentration $\mathrm{C}(\mathrm{x}, \mathrm{t})$ could then be applied as follows:

1. The initial concentration everywhere in cartilage is $0 \mathrm{mMol}$.

$$
\text { At } \mathrm{t}=0 \mathrm{~s} \quad \mathrm{C}(\mathrm{x}, 0)=0 \mathrm{mMol}
$$

2. The concentration in the reservoir is $1.0 \mathrm{mMol}$ and is constant and in the waterfilled chamber is $0 \mathrm{mMol}$.

$$
\text { For } \mathrm{t} \in[0, \infty] \quad \mathrm{C}_{\mathrm{b}}=1.0 \mathrm{mMol}
$$

3. The mass flux in the circumferential direction and the negative x-direction (cartilage to bone) is $0 \mathrm{mMol} / \mathrm{cm}^{2} \mathrm{~s}$. 
As a starting point the diffusivity was made to be the average diffusivity value of the corresponding experimental sample. The $\mathrm{R}$ and $\mathrm{D}$ values were then optimized using a nonlinear data fitting MATLAB ${ }^{\circledR}$ built in function, lsqcurvefit to minimize the difference between experimental and theoretical values. This function used the time-dependent experimental concentration data to best fit simulated concentration data by determining the optimum diffusivity value in cartilage and a resistivity value for the bone barrier. The simulation was repeated without the effect of the resistivity of bone. The error terms were compared from the optimization routines to show the improvement in the model due to the bone resistivity.

\subsection{Results}

The best-fit concentration distribution determined from the simulation in a typical specimen at specific times is shown for the model the included cartilage diffusivity and bone resistivity (Illustration 20) and for the model that included only cartilage diffusivity (Illustration 21). It can be seen qualitatively that including bone resistivity in the model provided much better fitted results. 


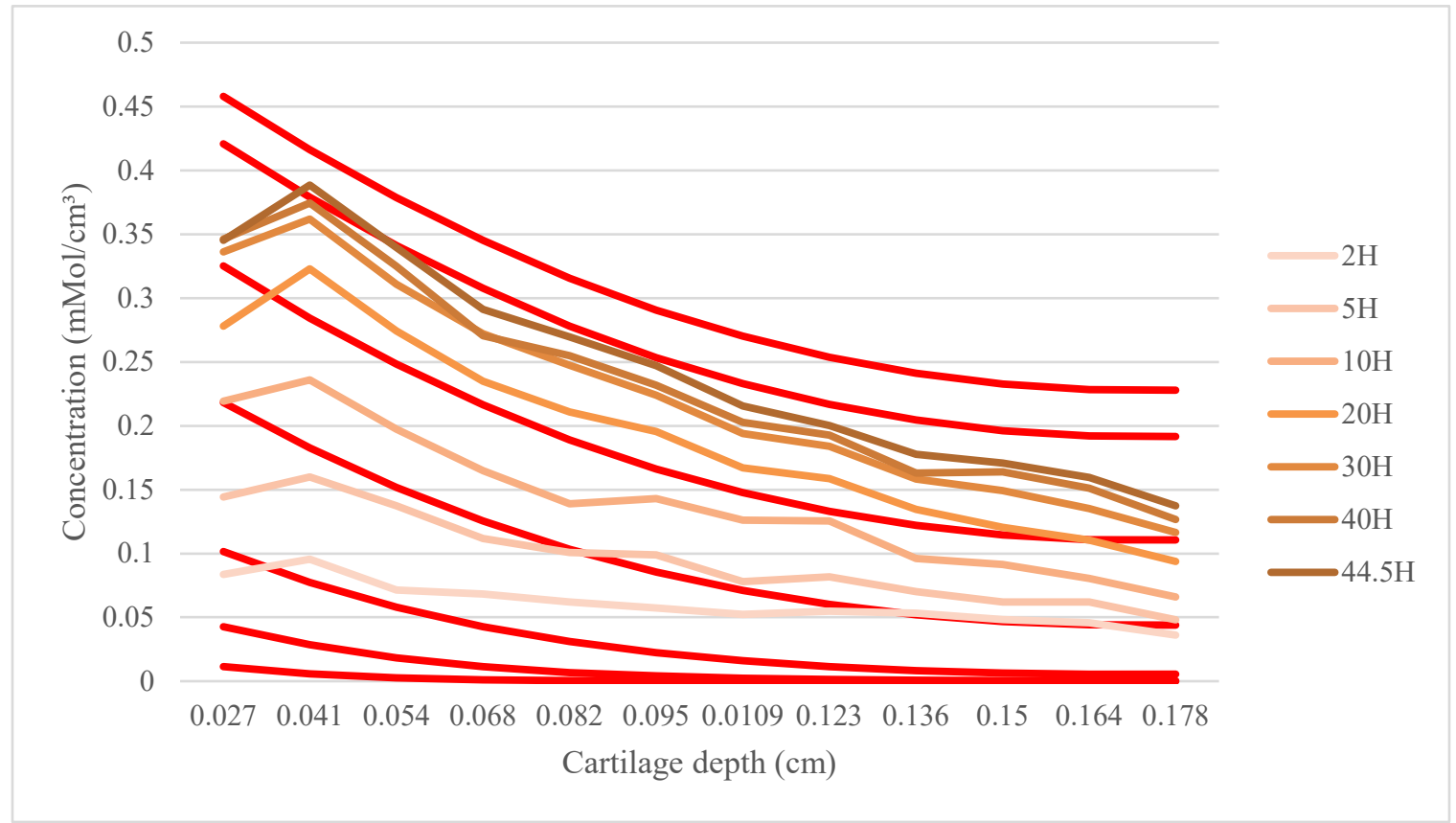

Illustration 20 Simulated best fit to sample 2 experimental concentrations. The simulation model included cartilage diffusivity and bone resistivity.

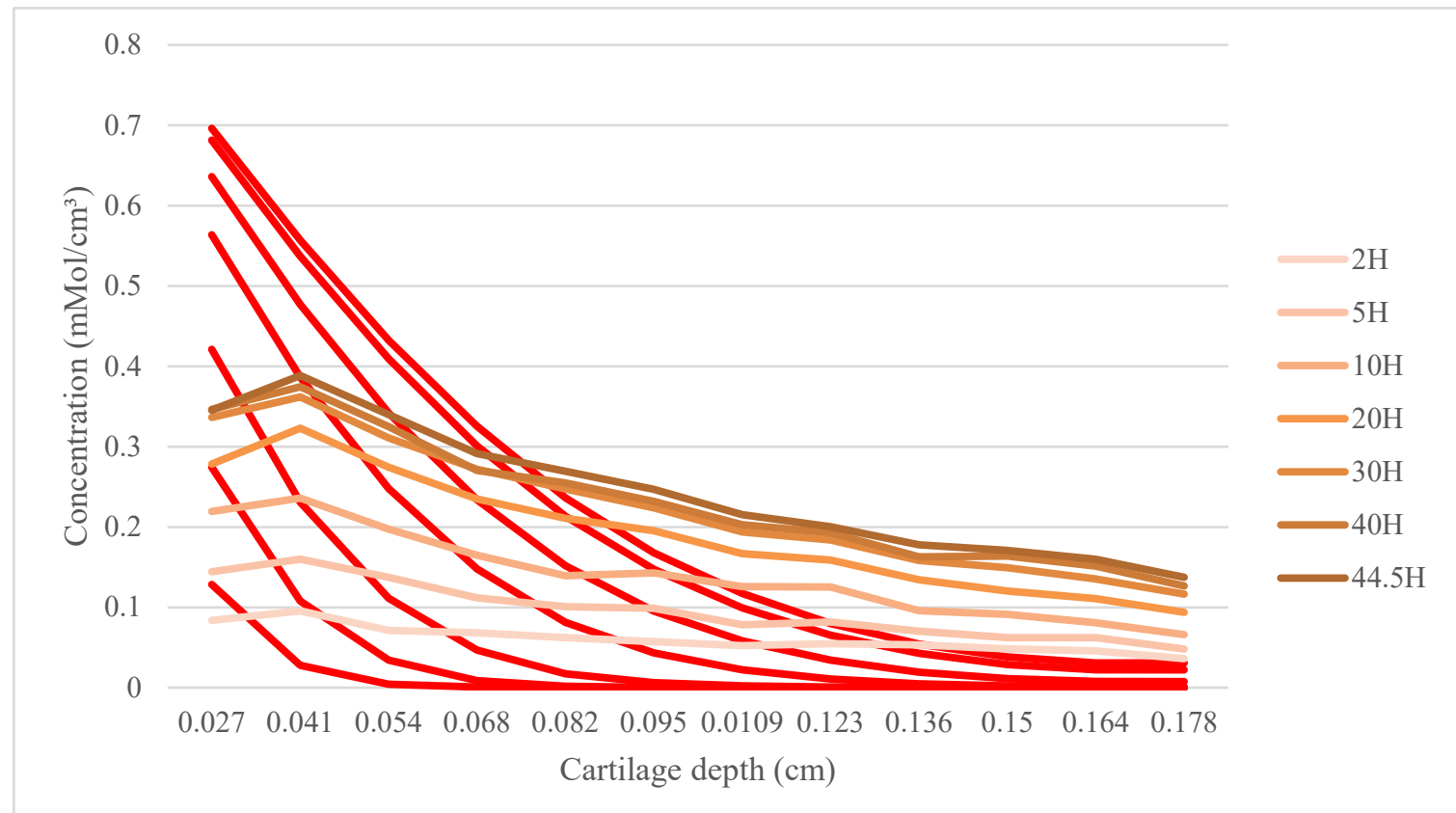

Illustration 21 Simulated best fit to sample 2 experimental concentrations. Bone resistivity was not included in the simulation model. 
Graphs for samples 1, 3-7 can be seen in appendices C and D (Illustrations 32-45).

Sample averaged diffusivity values of gadobutrol in cartilage were determined and compared to experimental values (see Table 7 and Illustration 19). The simulated calculations considered the resistivity of bone. For comparison, the root mean square (RMS) error and relative error are shown in Table 8. Relative error was calculated by dividing the RMS error by the concentration at the tidemark at the last time point. The large values of diffusivity obtained in the resistivity model (Table 8) occurred in specimens that did not display the typical monotonic decrease in concentration from the deep to the superficial zone. Rather, curves were typically flat (Appendix D) resulting in large best-fit diffusivity values.

Table 8 Experimental and simulated average diffusivity values at select times. Experimental values were determined in Chapter 2.

\begin{tabular}{|c|c|c|c|}
\hline \multirow{2}{*}{ 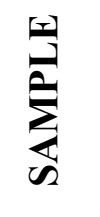 } & \multicolumn{3}{|c|}{ Diffusivity $\left(\times 10^{-6} \mathrm{~cm}^{2} / \mathrm{s}\right)$} \\
\hline & Experimental & With Resistivity & $\begin{array}{c}\text { Without } \\
\text { Resistivity }\end{array}$ \\
\hline 1 & 0.321 & 646 & 0.00236 \\
\hline 2 & 0.328 & 0.109 & 0.0149 \\
\hline 3 & 0.315 & 0.0281 & 0.00129 \\
\hline 4 & 0.207 & 3620 & 0.00156 \\
\hline 5 & 0.186 & 1230 & 0.00138 \\
\hline 6 & 0.144 & 0.0345 & 0.00532 \\
\hline 7 & 0.105 & 3.88 & 0.00109 \\
\hline AVG & 0.229 & 785 & 0.00398 \\
\hline
\end{tabular}


Table 9 RMS error and relative error of simulated concentrations with and without resistivity

\section{WITH RESISTIVITY WITHOUT RESISTIVITY}

\begin{tabular}{l|cccc}
\hline SAMPLE & $\begin{array}{l}\text { RMSE } \\
\left(\mathbf{m M o l} / \mathbf{c m}^{\mathbf{3}}\right)\end{array}$ & $\begin{array}{c}\text { relative } \\
\text { error } \mathbf{( \% )}\end{array}$ & $\begin{array}{c}\text { RMSE } \\
\left(\mathbf{m M o l} / \mathbf{c m}^{\mathbf{3}}\right)\end{array}$ & $\begin{array}{c}\text { relative } \\
\text { error } \mathbf{( \% )}\end{array}$ \\
$\mathbf{1}$ & 0.213 & 0.056 & 0.606 \\
$\mathbf{2}$ & 0.072 & 0.136 & 0.136 & 0.195 \\
$\mathbf{3}$ & 0.037 & 0.259 & 0.052 & 0.216 \\
$\mathbf{4}$ & 0.035 & 0.205 & 0.100 & 0.593 \\
$\mathbf{5}$ & 0.029 & 0.213 & 0.113 & 0.824 \\
$\mathbf{6}$ & 0.069 & 0.213 & 0.097 & 0.298 \\
$\mathbf{7}$ & 0.024 & 0.258 & 0.113 & 1.233 \\
\hline AVERAGE & $\mathbf{0 . 0 4 1}$ & $\mathbf{0 . 2 1 4}$ & $\mathbf{0 . 0 9 5}$ & $\mathbf{0 . 5 6 7}$ \\
& & & &
\end{tabular}

To determine the effect of bone on the diffusion of Gd into cartilage, the bone was modeled as a barrier. The $\mathrm{R}$ values for each sample can be seen in Table 9 .

Table 10 Best fit resisitivity values of each sample

\begin{tabular}{|r|r|}
\hline Sample & \multicolumn{2}{c|}{$\begin{array}{l}\text { Resistivity of } \\
\text { Bone, } R\left(\times 10^{-6} \mathbf{s}^{-1}\right)\end{array}$} \\
\hline 1 & 0.619 \\
2 & 5.00 \\
3 & 1.19 \\
4 & 0.573 \\
5 & 0.655 \\
6 & 2.89 \\
7 & 0.660 \\
\hline AVERAGE & 1.65 \\
\hline
\end{tabular}




\subsection{Discussion}

This study simulated the diffusion of gadobutrol in cartilage to determine the bone's resistance to diffusion of gadobutrol. Many assumptions were taken to simplify the model. Diffusivity in the highly porous trabecular subchondral bone was assumed to be high such that the initial concentration could be assumed $\mathrm{C}_{\mathrm{b}}$ everywhere. However, a dense but thin layer of subchondral bone is less permeable and was included in the current model as R (see Illustration 19). The exact thickness of the irregular subchondral bone layer is difficult to measure and changes with degeneration. The model implementation simplified the effect of the bone layer to a single resistivity value. Diffusivity is not a function of time, however was assessed at specific times. Diffusivity is a property of the material and so it was assumed that the properties of cartilage, such as the diffusivity, was uniform throughout the experiment. However, the assumption that the diffusivity is consistent throughout the cartilage depth may be incorrect as it has been confirmed that the properties differ in each zone. This can also be confirmed looking at experimental results (see Appendix C). The simulations do not exhibit this behavior as the assumption of constant diffusivity is implied when in fact diffusivity may vary due to the collagen network or concentration of GAG molecules.

As previously mentioned, it is highly likely that chemical or steric interactions between Gd and the ECM resulted in variations in diffusivity through the tissue depth which were not accounted for in the simulations. This would locally affect the rate of diffusion as well as the concentrations at equilibrium. Although Gadovist ${ }^{\circledR}$ is not a charged contrast agent, it is anticipated that comparable contrast agents with a charge such as Magnevist ${ }^{\circledR}$ would have different diffusivities. Diffusion would also largely depend on the 
size of the molecule. The larger the molecule, the lower the diffusion rate and there is likely a molecular weight threshold for diffusion across the osteochondral junction. In future work it would be valuable to examine the relationship of resistance to molecular weight of the solute being diffused.

In this study, only six to eight time points were selected. It is expected that higher number of time points would have resulted in a better fitted model, particularly the earlier times as the simulated values were very different in comparison to the experimental values and the optimization seemed to be less accurate.

The first experiment took into account the resistivity and optimized both the resistivity of bone and diffusivity of $\mathrm{Gd}$ by curve fitting the concentration to the experimental concentrations in cartilage (see Appendix C). The second experiment was conducted in the same manor, but did not consider the resistivity and simulated an experiment where the bone would be entirely removed and the Gd reservoir would be exposed directly to cartilage (see Appendix D). In Table 8, it can be seen that the average RMS error and relative error is lower when the resistivity of the bone barrier was taken into account. This aligns with the experimental part of this thesis (Chapter 2), which left some bone on the osteochondral plug. This was also confirmed in the images of the osteochondral plug where the thin layer of subchondral bone remained black in the images. In future experiments, it would be useful to experimentally remove the bone and try to simulate the results for comparison. Conversely, the cartilage could be removed and the bone resistivity measured directly.

It was assumed that the Gd concentration in the reservoir above the bone remained constant, however after converting the signal intensity of the image of the Gd reservoir at 
all times, it could be seen that the concentration was in fact decreasing by $22 \%$ to $50 \%$. Because there was a finite amount of Gd in the reservoir it was assumed that as the $\mathrm{Gd}$ distribution reaches equilibrium the concentration in the reservoir will decrease. To determine the effect of the changing concentration on the diffusivity value, the simulation was run for samples 2,3 and 6 while applying the experimental values of Gd concentration in the reservoir. The diffusivity value changed from $0.109 \mathrm{E}-06 \mathrm{~cm}^{2} / \mathrm{s}$ to $0.123 \mathrm{E}-06 \mathrm{~cm}^{2} / \mathrm{s}$ and the resistivity from $5.03 \mathrm{E}-06 \mathrm{~s}^{-1}$ to $2.21 \mathrm{E}-06 \mathrm{~s}^{-1}$., i.e. changes of $13 \%$ and $44 \%$, respectively. These differing values in $\mathrm{D}$ and $\mathrm{R}$ would indicate that the assumption of constant Gd concentration is incorrect and affects the accuracy of these results. Because samples were not imaged until equilibrium is reached, the final concentration of Gd in the reservoir once equilibrium was reached in the sample, could not be determined. The time to reach equilibrium is expected to vary based on the physical properties of the sample, such as the resistivity of the bone as well as the volume of the bone.

A sensitivity analysis was done to determine the impacts of changing parameters on resistivity and diffusivity. The parameter change that had the greatest impact on resistivity was an increase in the concentration of gadobutrol in the reservoir. When this concentration was doubled, the resistivity decreased by $43 \%$ and the diffusivity slightly increased by $7 \%$. Changing the number of time steps or the number of ROIs by a factor of $1 / 2$ and 2 had no impact on the resistivity or diffusivity, when increased by a factor of 10 , there was a very slight increase in diffusivity $(2 \%)$ and resistivity (1\%).

To better understand diffusivity of external solutes in joints, it would be of value to assess the role of dynamic joint loading on the rate of diffusion across the osteochondral junction by simulating time-varying pressure gradients or fluid flow as a forcing function 
to assess role of joint loading in diffusion of nutrients and biochemical signals. Future studies using a more complex FD or a finite element model without the simplifications made in this study would provide more accurate results. Having theoretical values of resistivity, it would be important to have experimental results for comparison. Experimentally, to assess the impact of resistivity, the bone could be removed entirely before diffusing a solute. This could be done applying the method described in Chapter 2 with an MR scanner. Alternatively, this could also be measured by diffusing a solute with quantifiable properties, i.e. acidity $(\mathrm{pH})$ or salinity. A mild acid, that would not affect the bone mineral, could be diffused across the osteochondral junction and quantified using a pH meter on the cartilage side.

In conclusion, the experimental results could be reproduced applying 1D diffusion theory and FD analysis. Through this method a resistance value could be assigned to the bone and the average was found to be $0.48 \times 10^{6} \mathrm{~cm}^{2} / \mathrm{s}$. The inclusion of $\mathrm{R}$ in the FD model improved the results predicted by the 1D diffusion model. 


\section{Chapter: Fluorescence Spectroscopy}

\subsection{Introduction}

Articular cartilage is avascular, aneural and alympthatic tissue that must receive nutrients and other molecules necessary for tissue function. Extracellular matrix comprises approximately $90 \%$ of cartilage volume with collagen representing approximately $60-70 \%$ of the dry weight [17]. The fluidic phase of cartilage is largely composed of proteoglycans [16]. Proteoglycans are highly anionic and are determinants in the distribution of solutes as they attract various cations, including water and repulse anions [16]. Chondrocytes are cells found in cartilage that are responsible for maintaining homeostasis and give rise to the mechanical properties of cartilage. Cartilage structure differs in organisms and even changes throughout the depth of the tissue [26], including changes in the orientation of collagen in each of the three zones [15-16]. As shown in Chapter 3 and in previous studies, this may result in heterogeneous and anisotropic diffusivity. Chondrocytes exhibit improved viability when bone tissue is present in the culture media but the mechanism remains unclear [14]. How the bone affects the cartilage is not yet proven, but it has been shown in a previous study by Arkill and Winlove and in Chapter 2, that small molecular weight solutes can passively diffuse across the osteochondral junction [26]. It is not yet clear if larger molecules, such as cytokines (see Table 2) can diffuse across the osteochondral junction. It has been established that larger molecules experience large steric interactions which could have an impact on their ability to diffusive passively [26]. Larger molecules that fluoresce when exposed to light of a specific wavelength may be used as tracer molecules to assess diffusion in microscope sections. Contrast-enhanced MRI with 
large molecules is not possible since available gadolinium-based compounds all have low molecular weight.

\subsubsection{Fluorescence and Fluorescent Microscopy}

Luminescence is the emission of light from any substance [103].

Photoluminescence is a type of luminescence that refers to a species absorbing light (photons) causing a state of electron excitation followed by light emission as the species de-excites [104]. Photoluminescence can further be divided into the fluorescence and phosphorescence $[103,104]$. The difference between these two is the time between absorption and emission [103-105]. Fluorescence emission occurs within $10^{-8}$ seconds, while phosphorescence emission times are typically between $10^{-3}$ and 1 seconds [103]. Fluorescence is not visible to the human eye and can only occur if the excitation light wavelength is in the ultraviolet region of the light spectrum [105]. Molecules exhibiting fluorescence, known as fluorophores, have long been employed to observe physical or chemical processes and structures in biological studies [104]. To study structure and physical parameters, a specific type of fluorophore, known as a fluorescent probe is employed [104]. Fluorophores each have a very specific range of excitation wavelengths required to induce a state of excitation and similarly have a specific range of emission wavelengths that are independent of excitation wavelength (Kasha's rule) [103, 104]. Many biological substances have primary fluorescence, also known as autofluorescence [103]. This is particularly important for the current study as bone and cartilage emit some autofluorescence [106]. This is largely due to the collagen and osteons [106-108]. In bone, the older osteons fluoresced the most and the new ones the least [106]. The autofluorescence could not be corrected by demineralizing the bone, and further to this, 
using solvents also had no effect [106, 107]. The autofluorescence in bone was not uniform, but varied with the varied concentration of collagen and varied age of osteons [106].

Fluorescent microscopes use xenon, mercury or metal halide arc lamp to produce ultraviolet light $[109,110]$. The light source is then filtered using a dichroic mirror, which reflects one range of wavelength and allows another to pass [109, 110]. Excitation and emission filters are also positioned in the optical path [109]. The emission filter is required to excite the fluorophore at its specified excitation wavelength and causes the fluorophore to emit light at a specific wavelength which passes through the emission filter before being detected by the optical lens or displayed on a computer software using a camera [110]. A large variety of fluorophores with different properties are available. Fluorophores can even be synthesized to meet specific needs. In Chapter 2, Gadovist was chosen as a diffusion tracer because it was readily available and had a molecular weight (MW) comparable to nutrients. Unfortunately, synthesizing MR contrast agents is not straightforward and there were no MR contrast agents with high MW. Thus, an alternative method was required to study the diffusion of larger molecules. This study set out to demonstrate that larger neutral solutes, up to $70 \mathrm{kDa}$ can passively diffuse across the osteochondral junction in human specimens. Fluorophores with comparable MW to the biomarkers listed in Table 2 are readily available. We also hypothesized that the diffusivity changes with degeneration of the osteochondral joint. 


\subsection{Methods}

\subsubsection{Sample Preparation}

The orthopedic surgical team at the Ottawa Hospital General Campus performed knee and hip replacement surgeries on ten patients. The patients were undergoing hip replacement surgery due to osteoarthritis or femoral neck fracture and were both females and males above the age of 50. As it is impossible to obtain healthy, living samples, for this study, the samples that were collected from hip trauma (fracture) patients were considered to be healthy control specimens. A total of ten femoral heads were collected, six from OA patients and four from femoral neck fracture patients. This study was reviewed and approved by the Ottawa Hospital Research Ethics Board (OHSN-REB, protocol 20150444). All patients provided informed consent prior to being enrolled in the study. From each femoral head, four osteochondral plugs were removed using a $10 \mathrm{~mm}$ trephine. The plugs were cylindrical, with a diameter of $10 \mathrm{~mm}$ and approximately $4 \mathrm{~mm}$ thickness, depending on the cartilage thickness. Once the bone was trimmed, the samples were fixed into plastic washers using quickset epoxy for use in the diffusion chamber described in Chapter 2. The bottom chamber of the diffusion chamber was filled with distilled water to prevent the cartilage from drying. Once the epoxy had hardened the washers containing the samples were placed into the middle of the diffusion chambers creating a separation between the top and bottom chambers (refer to Illustrations 3 and 4). The washer was sealed using marine-grade silicone and allowed to dry for 20 minutes. Once the silicone had dried, the top chamber was filled with a mixture of distilled water and fluorescent tracer $(0.10$ $\mathrm{mg} / \mathrm{mL}$ ). The 3 tracers selected for this study were fluorescein isothiocyanate-dextran (FITC) FD4 (Sigma-Aldrich, MW 3-5 kDa), dextran Texas Red® (Thermo Fisher 
Scientific, MW $10 \mathrm{kDa}$ ) and FITC FD70S (Sigma-Aldrich, $70 \mathrm{kDa}$ ). Molecular weight, excitation and emission information for the selected probes are summarized in Table .

Table 11 Excitation and emission values for fluorescein and Texas Red [111, 112]

\begin{tabular}{|l|l|l|l|}
\hline & $\begin{array}{l}\text { Molecular } \\
\text { Weight (kDa) }\end{array}$ & $\begin{array}{l}\text { Excitation } \\
(\mathbf{n m})\end{array}$ & $\begin{array}{l}\text { Emission } \\
(\mathbf{n m})\end{array}$ \\
\hline FITC FD4 & $3-5$ & 490 & 520 \\
FITC FD70s & 70 & 490 & 520 \\
Texas Red ${ }^{\circledR}$ & 10 & 595 & 615 \\
\hline
\end{tabular}

Table 10 shows the tracer selected, the state of the sample and the selected times for each specimen. The times were selected based on the Arkill and Winlove study, for comparison purposes [26].

Table 12 Sample and tracer information for fluorescent tracer diffusion experiments

\begin{tabular}{|c|l|l|l|}
\hline Specimen & OA or control & Tracer & Exposure times \\
\hline $\mathbf{1}$ & OA & FD4 & $2,5,17$ hours \\
\hline $\mathbf{2}$ & OA & FD70S & $2,5,17,160$ hours \\
\hline $\mathbf{3}$ & Control & FD4 & $2,5,17,160$ hours \\
\hline $\mathbf{4}$ & OA & FD4 & $2,5,17,160$ hours \\
\hline $\mathbf{5}$ & OA & FD70S & $2,5,17,160$ hours \\
\hline $\mathbf{6}$ & Control & FD70S & $2,5,17$ hours \\
\hline $\mathbf{7}$ & Control & Texas Red $\AA$ & $2,5,17,160$ hours \\
\hline $\mathbf{8}$ & Control & Texas Red $\AA$ & $2,5,17,160$ hours \\
\hline $\mathbf{9}$ & OA & Texas Red $\AA$ & $2,5,17,160$ hours \\
\hline $\mathbf{1 0}$ & OA & FD4 & 98 hours \\
\hline & & \multicolumn{2}{|l}{} \\
\hline
\end{tabular}


Because of the destructive nature of these tests, specific times were pre-selected: $2,5,17$ and 160 hours. A set of four osteochondral plugs was prepared from each patient, with each plug exposed to the fluorescent tracer for a different exposure time among the pre-selected times. The last time point was selected as it was expected that equilibrium would be reached at this time. Once the sample was exposed for the appropriate amount of time, it was removed from the diffusion chamber and placed in 10\% Formalin Fixative for 2 hours as per lab biosafety requirements. The sample was then embedded in optimal cutting temperature (OCT) compound and quick frozen using isopropane surrounded by dry ice. Once frozen, the embedded sample was then brought to a cryostat (Microtome Cryostat Microm HM, Thermo Scientific ${ }^{\mathrm{TM}}$ ) to be frozen onto a chuck using OCT compound to freeze-bind it. The chuck and sample were placed in the cryostat stage and five transverse sections with thicknesses of 30 microns were cut and placed onto slides. Once mounted onto the slide, VECTASHIELD ${ }^{\circledR}$ (Vector Laboratories), an antifade mounting medium was applied to cover the sample before placing a cover slip on top. To prevent leakage of VECTASHIELD® a clear nail polish was applied to the edges of the cover slip and allowed to dry before bringing to the microscope. This procedure was repeated for each sample at each exposure time.

\subsubsection{Imaging and Image Analysis}

Once the slides were ready, they could be observed using a confocal microscope (Zeiss LSM880 AxioObserverZ1). The microscope selected had filters specifically for

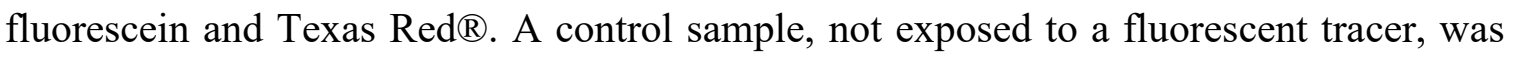
imaged as a reference and demonstrated autofluorescence across the ultraviolet spectrum, 
except for the far-red. For reference, the microscope software was used to measure the intensity of the autofluorescence.

\subsection{Results}

Sample 1, which was exposed to FD4 for 17 hours was imaged under microscope (Illustration 23). It was not visibly apparent that the tracer was present in the cartilage or the bone. Using the intensity value of the unexposed control sample as a reference (Illustration 22), the brighter areas of the Sample 1 image were measured to be approximately 200 relative florescence units (RFU) and were found to be approximately the same value as the control sample.

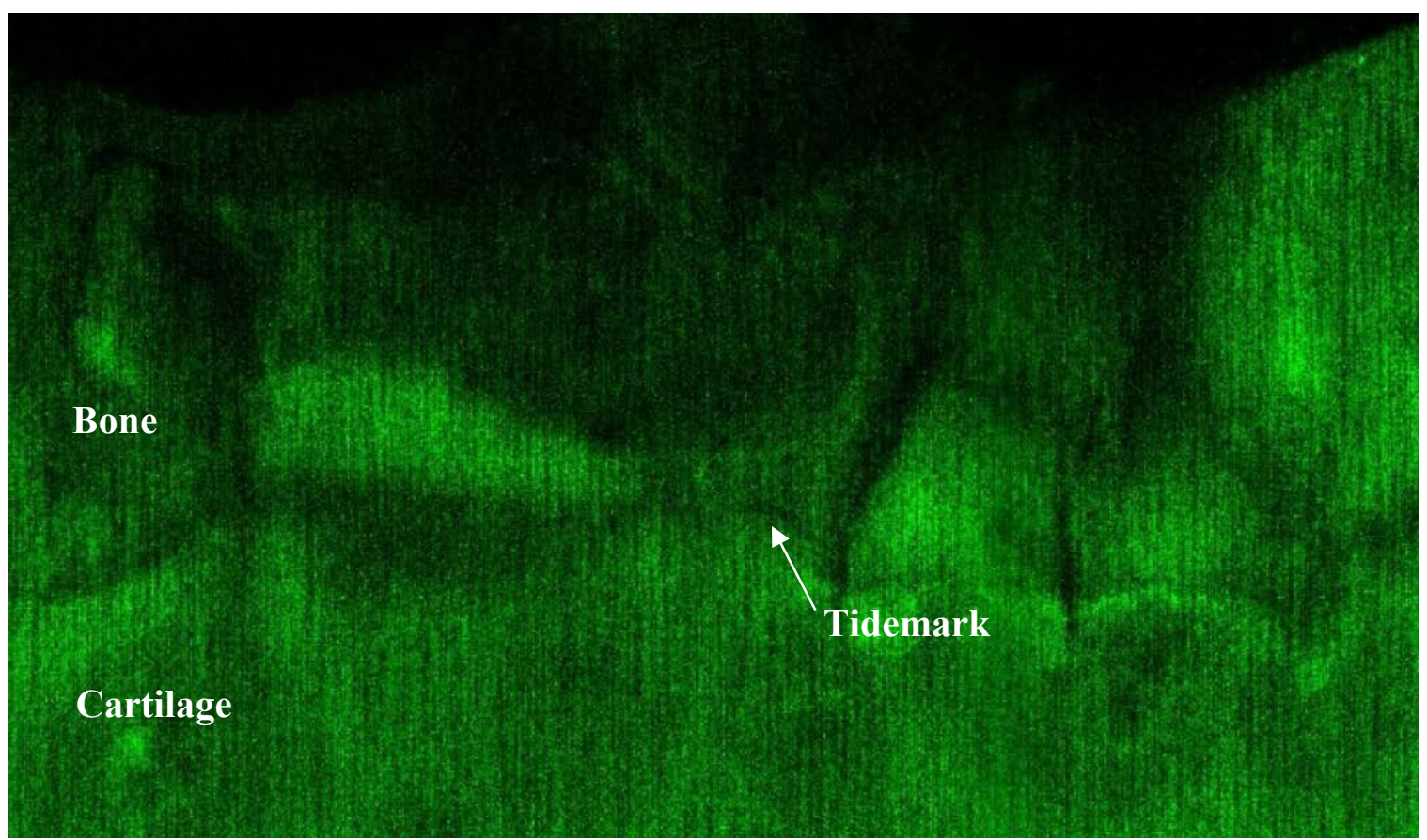

Illustration 22 Fluorescent Microscope image of an unexposed sample 


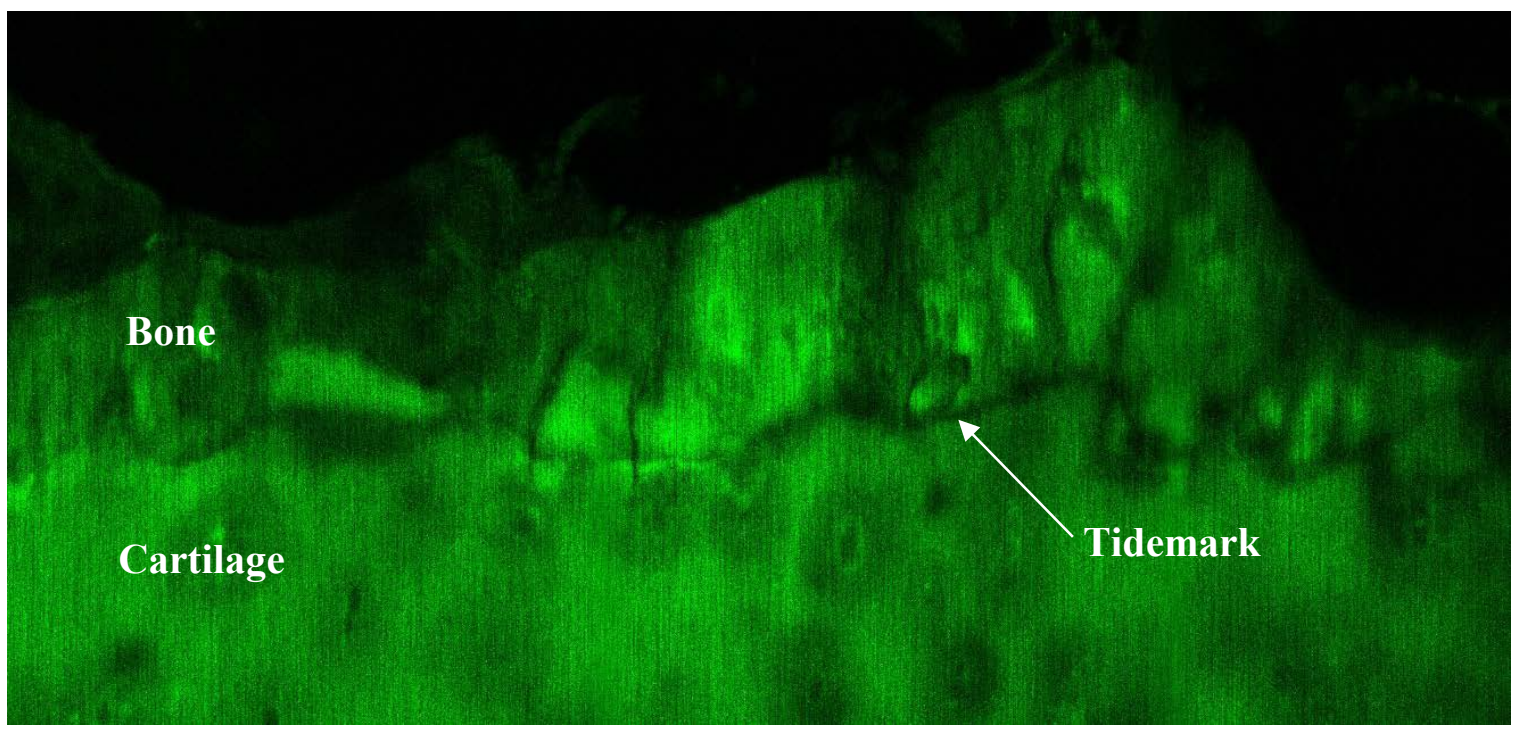

Illustration 23 Sample 1 exposed to FITC FD4 for 17 hours

Sample 2 which had been exposed to the largest fluorophore, FD70S also did not demonstrate any evidence of tracer in the bone or cartilage. A variety of ombinations with tracer and osteochondral health status were attempted (see Table 7). These results indicate that either the autofluorescence is masking the fluorescein tracer fluorescence, or no tracer could diffuse from the bone side into the cartilage.

To verify the tracer was not faulty, a small amount of diluted tracer was placed on a slide to observe under microscope. The images produced showed very high fluorescence intensity with values of approximately 1000 RFU and when compared to the intensity of the autofluorescence (approximately 200 RFU) of the control sample, it became obvious that the bright parts of the image of sample 1 were autofluorescence and not an accumulation of the tracer. Because of these results, it was decided to try and expose tracer directly to the cartilage to remove the added barrier to diffusion that is bone. A slight increase in image intensity could be seen at the tidemark location (see Illustration 24) but was inconclusive in confirming that the fluorescein had successfully diffused into the cartilage up to the tidemark. 


\section{Bone}

\section{Tidemark}

\section{Cartilage}

Illustration 24 Sample 10 cartilage exposed directly to Fluorescein FD4 for 98 hours

A summary of all results can be seen in Table 13 .

Table 13 Summary of findings for Fluorescent experiment (Specimens 1-10)

\begin{tabular}{|c|c|c|c|c|}
\hline Specimen & $\begin{array}{l}\text { Healthy } \\
\text { or OA }\end{array}$ & Tracer & $\begin{array}{l}\text { Exposure Times } \\
\text { (Hours) }\end{array}$ & Results \\
\hline 1 & $\mathrm{OA}$ & FD4 & $2,5,17$ & Negative for tracer \\
\hline 2 & $\mathrm{OA}$ & FD70S & $2,5,17,160$ & Negative for tracer \\
\hline 3 & Control & FD4 & $2,5,17,160$ & Negative for tracer \\
\hline 4 & $\mathrm{OA}$ & FD4 & $2,5,17,160$ & Negative for tracer \\
\hline 5 & $\mathrm{OA}$ & FD70S & $2,5,17,160$ & Negative for tracer \\
\hline 6 & Control & FD70S & $2,5,17$ hours & Negative for tracer \\
\hline 7 & Control & Texas Red $\AA$ & $2,5,17,160$ & Negative for tracer \\
\hline 8 & Control & Texas Red® & $2,5,17,160$ & Negative for tracer \\
\hline 9 & $\mathrm{OA}$ & Texas Red ( & $2,5,17,160$ & Negative for tracer \\
\hline 10 & $\mathrm{OA}$ & FD4 & 98 & Negative for tracer \\
\hline
\end{tabular}

\subsection{Discussion}

The purpose of this experiment was to reproduce Arkill's experiment but with larger MW solutes analogous to biochemical signal molecules such as cytokines. The tracers used in this study ranged from 3 to $70 \mathrm{kDa}$ to cover the range of biomarkers 
indicated in Table 2 (Chapter 1), namely TNF $\alpha$ (MW $26 \mathrm{kDa}$ ) and IGF (7.65 kDa). Initially, it was unclear if any of the probes were successfully reaching the cartilage as the image intensity was very similar to the unexposed control sample. After observing multiple samples at the four selected time points, it was evident that no tracer was passing through the bone. There are a number of hypotheses as to why the tracer was not able to diffuse into the bone. In one study by Frederico and Herzog, they believed that convective transport induced by cyclic compressive loading is important for larger solutes [95]. Human joints are constantly under cyclic compressive loading due to everyday movements which could play an essential role in diffusing larger molecules such as chondrocytes. In another study by Sterner et al., size and charge of molecule were crucial properties for retention in cartilage [115]. This study found that positively charged molecules with MW up to $233 \mathrm{kDa}$ successfully diffused and were retained in the negatively charged cartilage [115]. In an earlier study, cartilage's ability to intake solutes decreased steeply with increasing protein size [81]. Using a different approach, a study conducted by Walter, et al., TNF $\alpha$ with fluorescent markers were diffused into the nucleus pulposis of the intervertebral disc, a tissue very similar to articular cartilage, by static and dynamic loading, resulted in significantly greater amount of TNF $\alpha$ in the samples that had been dynamically loaded [118]. Similarly, when dynamically loading articular cartilage to aid the diffusion of IGF-1 into articular cartilage, the uptake was significantly faster (less than half the time) than the control tissue [119]. In a study conducted by Arkill, in-tact equine joints were perfused with small, nutrient-sized probes, rhodamine (MW 443 Da) and fluorescein (MW $332 \mathrm{Da}$ ), but there was no increase in tracer uptake due to loading compared to unloaded specimens [26]. In summary, many studies have concluded that 
dynamic loading resulted in higher rates of diffusion, for both small solutes and larger solutes $[26,95,115-119]$.

It is also believed that the diffusion of external solutes depends on two mechanisms, steric interactions caused by glycosaminoglycans and collagen fibers, and electrostatic interactions due to the glycosaminoglycans fixed charge density with the solutes [113]. Increase in proteoglycan concentration is reported to cause a negative steric interaction with molecules, particularly larger molecules [102]. In another study by Salo et al. a negatively charged small, external solute was diffused by creating a chemical gradient [114]. This study examined the permeability of cartilage to large molecules and concluded that diffusion of solutes is extremely sensitive to glycosaminoglycan content of the cartilage [81]. In future studies this in-tact limb experimental setup could be applied in attempt to drive the diffusion of larger solutes. Alternatively, a pressure gradient could be applied to the osteochondral plug itself to reduce the diffusion time.

This study demonstrated that larger neutral solutes, as little as 3-5 kDa could not passively diffuse across the osteochondral junction in human specimens. It was unclear if the fluorescein, FD4 did in fact diffuse passively when exposed directly to the cartilage surface. Future studies, with longer exposure times could confirm these results.

Limitations include low number of samples for practical reasons. As the rate of data collection was largely dictated by patient consent, only a limited number of samples could be collected in the allowed timeframe. It was also extremely difficult to cut the samples while maintaining the structural integrity of the bone. In almost all slide-mounted samples the bone structure was shifted or broken due to folds in the OCT, lifting of the sample 
during thawing or VECTASHIELD ${ }^{\circledR}$ causing parts of the sample to float or become mobile. This can be observed in Illustrations 22 to 24 .

In conclusion, larger solutes with molecular weights ranging between 3 and $70 \mathrm{kDa}$ could not passively diffuse across the osteochondral junction. Passive diffusion of fluorescein FD4 when exposed directly to the superficial cartilage was inconclusive. Future studies on diffusion of larger solutes are recommended to focus on forced diffusion of larger solutes. 


\section{Chapter: Discussion and Conclusions}

This thesis studied the molecular diffusion of Gadovist ${ }^{\circledR}$, as well as two fluorescein and two Texas Red fluorescent tracers. Molecular diffusion of gadobutrol-based contrast agent across the osteochondral junction could be measured using contrast-enhanced MR imaging. Fluorescent tracer diffusion could not be measured through fluorescent microscopy and further work is required to develop a valid test protocol. Simulation of the gadobutrol experiment demonstrated the effects of bone as barrier on the diffusion of solutes across the osteochondral junction.

Tissue-averaged diffusion coefficients for gadobutrol were determined and experimental results were reproduced using FD modeling. Although equilibrium was not yet reached after 60 hours of continuous imaging the rate of diffusion could be seen to decrease in the last 10 hours of imaging. The time-dependent distribution of gadobutrol seemed to reflect the difference in cartilage microstructure for each of the zones. Although the uptake of Gd in the different zones in cartilage were not compared directly, it could be seen in the graphs shown in Appendix B, that the concentration of Gd may demonstrate an inverse relationship with the concentration of proteoglycans agreeing with previous studies $[65,66]$. Furthermore, diffusion occurred parallel to collagen fibers in the deep zone, but perpendicular to fibers in the superficial zone. Further work is required to determine quantitative relationships between gadobutrol diffusion and collagen orientation and proteoglycan concentration.

By reproducing experimental results through FD analysis, resistivity values of the osteochondral junction, as a barrier, could be determined. By altering the resistance value, it could be seen that the osteochondral junction acts as a barrier to diffusion and likely 
decreases the molecular diffusion rate. Degenerative changes in OA such as cracks in, or thickening of, the calcified osteochondral junction [27] may affect the resistivity of the osteochondral junction to solute diffusion. Investigation of this in arthritic specimens may reveal a new mechanism in osteoarthritic degeneration.

In the diffusion of fluorescent tracers, it was not possible to determine diffusivity values for larger molecules $(3-70 \mathrm{kDa})$ as it appeared the tracers were unable to cross the osteochondral junction. The low permeability of cartilage may require much longer diffusion times for larger molecules. It is believed that a forced diffusion, created through pressure, fluid flow, or use of cationic tracers would influence the diffusion of larger solutes.

\subsection{Contributions}

The main contributions of this thesis are summarized as follows:

1. Developed a protocol for measuring diffusion of an MR contrast agent through osteochondral samples. This protocol allows non-destructive study of diffusion processes and could be implemented in vivo.

2. Applied a numerical simulation of the diffusion of solutes in osteochondral tissues to assess the effect of the compact bone layer as a barrier to diffusion.

3. Demonstrated that neutral solutes with molecular weight greater than $3000 \mathrm{Da}$ are not able to passively diffuse across the osteochondral junction. 


\section{References}

[1] Bombardier, Claire, et al. "The Impact Of Arthritis In Canada: Today And Over The Next 30 Years" Arthritis Alliance of Canacer, Toronto, Canada, 2011.

[2] Badley, Elizabeth M. "Arthritis in Canada: What do we Know and what should we Know?" Journal of Rheumatology, vol. 32, no. 72, 2005, pp. 39-41.

[3] Sandell, Linda J. "Etiology of Osteoarthritis: Genetics and Synovial Joint Development." Nature Reviews Rheumatology, vol. 8, no. 2, 2012., pp. 77-89.

[4] Gupta, Sanjay, et al. "The Economic Burden of Disabling Hip and Knee Osteoarthritis (OA) from the Perspective of Individuals Living with this Condition." Rheumatology, vol. 44, no. 12, 2005., pp. 1531-1537.

[5] Jordan, Joanne M., Virginia B. Kraus, and Marc C. Hochberg. "Genetics of Osteoarthritis." Current Rheumatology Reports, vol. 6, no. 1, 2004., pp. 7-13.

[6] Mow, Van C., and Rik Huiskes. Basic orthopaedic biomechanics \& mechanobiology, 3d Ed. Vol 29. Lippincott Williams \& Wilkins, Portland, 2005.

[7] Hasler, Evelyne M., et al. "Articular cartilage biomechanics: theoretical models, material properties, and biosynthetic response." Critical reviews in biomedical engineering. Vol. 27, no. 6, 1999, pp. 415-488.

[8] Kellgren, Jonas and Lawrence, J.S. "Radiological Assessment of Osteo-Arthrosis." Annals of the rheumatic diseases, vol. 16, no. 4, 1957., pp. 494-502.

[9] Litwic, Anna, et al. "Epidemiology and burden of osteoarthritis." British medical bulletin. Vol. 105, no.1, 2013, pp. 185-199.

[10] Moskowitz, Roland W. "The burden of osteoarthritis: clinical and quality-of-life issues." The American journal of managed care, vol. 15, no.8, 2009, pp. S223-9.

[11] Pavelka, Margit and Roth, Jürgen. "Articular Cartilage." Functional Ultrastructure: An Atlas of Tissue Biology and Pathology. Springer Vienna, Vienna, 2005, pp. $262-$ 263.

[12] Mobasheri, Ali, et al. "Articular Cartilage: Structure, Function, and Pathophysiology." Facilitative Glucose Transporters in Articular Chondrocytes: Expression, Distribution and Functional Regulation of GLUT Isoforms by Hypoxia, Hypoxia Mimetics, Growth Factors and Pro-Inflammatory Cytokines (2008): 5-13. 
[13] Shepherd, D. E. T., and B. B. Seedhom. "Thickness of human articular cartilage in joints of the lower limb." Annals of the rheumatic diseases 58.1 (1999): 27-34.

[14] Amin, A. K., et al. "Chondrocyte Survival in Articular Cartilage: The Influence of Subchondral Bone in a Bovine Model." Journal of Bone and Joint Surgery - Series $B$, vol. 91, no. 5, 2009., pp. 691-699.

[15] Martin, James A., and Joseph A. Buckwalter. "Articular Cartilage Biology." Sports Injuries. Springer Berlin Heidelberg, 2012. 685-692.

[16] Horvai, Andrew. "Anatomy and histology of cartilage." Cartilage Imaging. Springer New York, 2011. 1-10.

[17] Kapoor, Mohit, and Nizar N. Mahomed, eds. Osteoarthritis: Pathogenesis, diagnosis, available treatments, drug safety, regenerative and precision medicine. Springer, 2015.

[18] Wang, Christopher C., et al. "Optical Determination of Anisotropic Material Properties of Bovine Articular Cartilage in Compression." Journal of Biomechanics, vol. 36, no. 3, 2003., pp. 339-353.

[19] Flik, Kyle R., et al. "Articular cartilage." Cartilage Repair Strategies. Humana Press, 2007. 1-12.

[20] Madry, Henning, C. Niek van Dijk, and Magdalena Mueller-Gerbl. "The basic science of the subchondral bone." Knee surgery, sports traumatology, arthroscopy 18.4 (2010): 419-433.

[21] Castañeda, Santos, et al. "Subchondral Bone as a Key Target for Osteoarthritis Treatment." Biochemical pharmacology, vol. 83, no. 3, 2012., pp. 315-323.

[22] Roman-Blas, Jorge A., and Gabriel Herrero-Beaumont. "Targeting Subchondral Bone in Osteoporotic Osteoarthritis." Arthritis research \& therapy, vol. 16, no. 6, 2014., pp. 494-494.

[23] Radin, Eric, et al. "Effects of mechanical loading on the tissues of the rabbit knee." Journal of Orthopeadic Research 2(3) (1984): 221-34

[24] Goldring, Mary B., and Steven R. Goldring. "Articular cartilage and subchondral bone in the pathogenesis of osteoarthritis." Annals of the New York Academy of Sciences 1192.1 (2010): 230-237.

[25] Loeser, Richard F., et al. "Osteoarthritis: a disease of the joint as an organ." Arthritis \& Rheumatism 64.6 (2012): 1697-1707. 
[26] Arkill, K. P., and C. P. Winlove. "Solute transport in the deep and calcified zones of articular cartilage." Osteoarthritis and Cartilage 16.6 (2008): 708-714.

[27] Burr, David B., and Eric L. Radin. "Microfractures and microcracks in subchondral bone: are they relevant to osteoarthrosis?." Rheumatic Disease Clinics 29.4 (2003): 675-685.

[28] Tat, Steeve Kwan, et al. "Targeting subchondral bone for treating osteoarthritis: what is the evidence?." Best Practice \& Research Clinical Rheumatology 24.1 (2010): 51-70.

[29] Glyn-Jones, S., et al. "osteoarthritis." vol. 386, no. 9991, Lancet, London, England, 2015., pp. 376-387.

[30] Zhang, W., and M. Doherty. "EULAR recommendations for knee and hip osteoarthritis: a critique of the methodology." British journal of sports medicine 40.8 (2006): 664-669.

[31] Brandt, Kenneth D. "Osteoarthritis Diagnosis: Avoiding the Pitfalls." The Journal of Musculoskeletal Medicine, vol. 27, no. 11, 2010., pp. 445.

[32] Hunter, David J., Deborah Schofield, and Emily Callander. "The individual and socioeconomic impact of osteoarthritis." Nature Reviews Rheumatology 10.7 (2014): 437-441.

[33] March, Lynette M., and Clarissa JM Bachmeier. "10 Economics of osteoarthritis: a global perspective." Baillière's clinical rheumatology 11.4 (1997): 817-834.

[34] Goldring, Mary B., and Steven R. Goldring. "Osteoarthritis." Journal of Cellular Physiology, vol. 213, no. 3, 2007., pp. 626-634.

[35] Braun, Hillary J., and Garry E. Gold. "Diagnosis of Osteoarthritis: Imaging." Bone, vol. 51, no. 2, 2012., pp. 278-288.

[36] Brandt, Kenneth D., Paul Dieppe, and Eric L. Radin. "Etiopathogenesis of osteoarthritis." Rheumatic Disease Clinics of North America 34.3 (2008): 531-559.

[37] Dequeker, J., et al. "Generalized osteoarthritis associated with increased insulin-like growth factor types $i$ and ii and transforming growth factor $\beta$ in cortical bone from the iliac crest. possible mechanism of increased bone density and protection against osteoporosis." Arthritis \& Rheumatology 36.12 (1993): 1702-1708. 
[38] Dequeker, Jan, and F. P. Luyten. "The history of osteoarthritisosteoarthrosis." Annals of the rheumatic diseases 67.1 (2008): 5-10.

[39] Kapoor, Mohit, and Nizar N. Mahomed, eds. Osteoarthritis: Pathogenesis, diagnosis, available treatments, drug safety, regenerative and precision medicine. Springer, 2015.

[40] Bonnin, Michel, and Pierre Chambat, eds. Osteoarthritis of the Knee. Springer Science \& Business Media, 2008.

[41] Reynard, Louise N., and John Loughlin. "Genetics and epigenetics of osteoarthritis." Maturitas 71.3 (2012): 200-204.

[42] Anderson, A. Shane, and Richard F. Loeser. "Why is osteoarthritis an age-related disease?." Best Practice \& Research Clinical Rheumatology 24.1 (2010): 15-26.

[43] Lotz, Martin, and Richard F. Loeser. "Effects of aging on articular cartilage homeostasis." Bone 51.2 (2012): 241-248.

[44] Loeser, Richard F. "Age-related changes in the musculoskeletal system and the development of osteoarthritis." Clinics in geriatric medicine 26.3 (2010): 371-386.

[45] Bliddal, Henning, et al. "Osteoarthritis-a role for weight management in rheumatology practice: an update." Clinical obesity 1.1 (2011): 50-52.

[46] Oliveria, Susan A., et al. "Body weight, body mass index, and incident symptomatic osteoarthritis of the hand, hip, and knee." Epidemiology 10.2 (1999): 161-166.

[47] Wise, Barton L., et al. "The Association of Knee Shape with Sex: The Osteoarthritis Initiative." Arthritis \& Rheumatology 66 (2014): S791-S792.

[48] Boyan, Barbara D., et al. "Sex differences in osteoarthritis of the knee." Journal of the American Academy of Orthopaedic Surgeons 20.10 (2012): 668-669.

[49] Lohmander, L. Stefan, et al. "The long-term consequence of anterior cruciate ligament and meniscus injuries osteoarthritis." The American journal of sports medicine 35.10 (2007): 1756-1769.

[50] Heijink, Andras, et al. "Biomechanical considerations in the pathogenesis of osteoarthritis of the knee." Knee Surgery, Sports Traumatology, Arthroscopy 20.3 (2012): 423-435. 
[51] Jackson, B. D., et al. "Reviewing knee osteoarthritis-a biomechanical perspective." Journal of Science and Medicine in Sport 7.3 (2004): 347-357.

[52] Sandell, Linda J. "Biomarkers in osteoarthritis." HSS journal 8.1 (2012): 33-34.

[53] Goldring, Steven R., and Mary B. Goldring. "The role of cytokines in cartilage matrix degeneration in osteoarthritis." Clinical orthopaedics and related research 427 (2004): S27-S36.

[54] Moos, V., et al. "Regulation of expression of cytokines and growth factors in osteoarthritic cartilage explants." Clinical rheumatology 20.5 (2001): 353-358.

[55] Tat, Steeve Kwan, et al. "Modulation of OPG, RANK and RANKL by human chondrocytes and their implication during osteoarthritis." Rheumatology (2009): kep300.

[56] Hilal, George, et al. "Endogenous Prostaglandin E2 and Insulin-like Growth Factor 1 Can Modulate the Levels of Parathyroid Hormone Receptor in Human Osteoarthritic Osteoblasts." Journal of Bone and Mineral Research 16.4 (2001): 713-721.

[57] Lee, Sunggun. "Endogenous parathyroid hormone and knee osteoarthritis: a crosssectional study." International journal of rheumatic diseases (2015).

[58] Reboul, Pascal, et al. "Hepatocyte growth factor induction of collagenase 3 production in human osteoarthritic cartilage: Involvement of the stress-activated protein kinase/c-Jun N-terminal kinase pathway and a sensitive p38 mitogenactivated protein kinase inhibitor cascade." Arthritis \& Rheumatism 44.1 (2001): 73-84.

[59] Reboul, P., et al. "Hepatocyte growth factor in osteoarthritis: when bone and cartilage decide to have a chat." Arthritis research \& therapy 5 (2003): 1-2.

[60] Hunter, David J., et al. "Evolution of semi-quantitative whole joint assessment of knee OA: MOAKS (MRI Osteoarthritis Knee Score)." Osteoarthritis and Cartilage 19.8 (2011): 990-1002.

[61] Delgado-Calle, J., et al. "Wnt activity, osteocytes and sclerostin expression in hip fractures and osteoarthritis." Bone 48 (2011): S138-S139.

[62] Lee, Sonia, et al. "Scoring hip osteoarthritis with MRI (SHOMRI): a whole joint osteoarthritis evaluation system." Journal of Magnetic Resonance Imaging 41.6 (2015): 1549-1557. 
[63] Gardiner, Bruce S., et al. "Predicting knee osteoarthritis." Annals of biomedical engineering 44.1 (2016): 222-233.

[64] Lohrke, Jessica, et al. "25 Years of Contrast-Enhanced MRI: Developments, Current Challenges and Future Perspectives." Advances in therapy 33.1 (2016): 1-28.

[65] Choi, Jung-Ah, and Garry E. Gold. "MR imaging of articular cartilage physiology." Magnetic resonance imaging clinics of North America 19.2 (2011): 249-282.

[66] Li, Wei, et al. "Delayed contrast-enhanced MRI of cartilage: Comparison of nonionic and ionic contrast agents." Magnetic resonance in medicine 64.5 (2010): 1267-1273.

[67] Núñez, Montserrat, et al. "Quality of life after joint replacement for osteoarthritis." Aging Health 6.4 (2010): 481-494.

[68] Mow, Van C., and A. Ratcliffe. "Structure and function of articular cartilage and meniscus." Basic orthopaedic biomechanics 2 (1997): 113-177.

[69] Rosemann, Thomas, Gunter Laux, and Joachim Szecsenyi. "Osteoarthritis: quality of life, comorbidities, medication and health service utilization assessed in a large sample of primary care patients." Journal of orthopaedic surgery and research 2.1 (2007): 12 .

[70] Palo, Nishit, et al. "Effects of Osteoarthritis on Quality of life in Elderly Population of Bhubaneswar, India: A Prospective Multicenter Screening and Therapeutic Study of 2854 Patients." Geriatric orthopaedic surgery \& rehabilitation 6.4 (2015): 269275.

[71] Karina, L. "Determining the Quality of Life in Osteoarthritis Patients." Scandinavian Journal Of Rheumatology, vol. 41, 2012., pp. 56-56.

[72] Rutt, Brian K., and Donald H. Lee. "The impact of field strength on image quality in MRI." Journal of Magnetic Resonance Imaging 6.1 (1996): 57-62.

[73] Scher, Jose U. "Several approaches are combined with a focus on alleviating symptoms--Managing osteoarthritis: The treatment options." The Journal of Musculoskeletal Medicine 23.10 (2006): 701-701. 
[74] Bliddal, Henning, et al. "Weight loss as treatment for knee osteoarthritis symptoms in obese patients: 1-year results from a randomised controlled trial." Annals of the rheumatic diseases (2011): annrheumdis 142018.

[75] Warren, Ed, and G. P. Trainer. "Managing Osteoarthritic Pain." Practice Nurse, vol. 39, no. 12, 2010., pp. 16-19.

[76] Gahunia, Harpal K., and Kenneth P. H. Pritzker. "Effect of Exercise on Articular Cartilage." Orthopedic Clinics of North America, vol. 43, no. 2, 2012., pp. 187-199.

[77] Leopold, Seth S. "Minimally Invasive Total Knee Arthroplasty for Osteoarthritis." The New England Journal of Medicine, vol. 360, no. 17, 2009., pp. 1749-1758.

[78] Reichenbach, Stephan, et al. "Joint Lavage for Osteoarthritis of the Knee." Cochrane database of systematic reviews (Online), vol. 5, no. 5, 2010.

[79] Jones, G., et al. "Early radiographic osteoarthritis is associated with substantial changes in cartilage volume and tibial bone surface area in both males and females." Osteoarthritis and cartilage 12.2 (2004): 169-174.

[80] Comyn, John. "Introduction to polymer permeability and the mathematics of diffusion." Polymer permeability. Springer Netherlands, 1985. 1-10.

[81] Maroudas, A. "Transport of solutes through cartilage: permeability to large molecules." Journal of anatomy 122.Pt 2 (1976): 335.

[82] Arkill, Kenton P., and C. P. Winlove. "Fatty Acid Transport in Articular Cartilage." Archives of Biochemistry and Biophysics, vol. 456, no. 1, 2006., pp. 71-78.

[83] Crank, John. The mathematics of diffusion. Oxford university press, 1979.

[84] Davis, Craig, Genant, Harry and Dunham, Jeffery. "The Effects of Bone on Proton NMR Relaxation Times of Surrounding Liquids.” Investigative Radiology, vol. 21, 1986. pp. $472-477$

[85] Donahue, Kathleen M., et al. "Studies of Gd-DTPA Relaxivity and Proton Exchange Rates in Tissue." Magnetic resonance in medicine, vol. 32, no. 1, 1994., pp. 66-76.

[86] Gillis, Amy, Martha Gray, and Deborah Burstein. "Relaxivity and Diffusion of Gadolinium Agents in Cartilage." Magnetic Resonance in Medicine, vol. 48, no. 6, 2002., pp. 1068-1071. 
[87] Vignaud, Alexandre et al. "Comparison of marketed Gadolinium-based Contrast Agents Relaxivities on Clinical MR scanner at 1.5T, 3T and 7T in water and plasma for a large range of physiological concentrations." Proc. Intl. Soc. Mag. Reson. Med. Vol. 22. 2014

[88] Doty, F. David, et al. "Radio frequency coil technology for small-animal MRI." NMR in Biomedicine 20.3 (2007): 304-325.

[89] Plenge, Esben, et al. "Super-resolution methods in MRI: Can they improve the tradeoff between resolution, signal-to-noise ratio, and acquisition time?." Magnetic resonance in medicine 68.6 (2012): 1983-1993.

[90] Ioannidou, Evangelia. "Taphonomy of animal bones: species, sex, age and breed variability of sheep, cattle and pig bone density." Journal of Archaeological Science 30.3 (2003): 355-365.

[91] Lukas, Vanessa A., et al. "Classification of histologically scored human knee osteochondral plugs by quantitative analysis of magnetic resonance images at 3T." Journal of Orthopaedic Research 33.5 (2015): 640-650.

[92] Peterfy, Charles, and Manish Kothari. "Imaging osteoarthritis: magnetic resonance imaging versus x-ray." Current rheumatology reports 8.1 (2006): 16.

[93] Roemer, Frank W., et al. "The role of imaging in osteoarthritis." Best practice \& research Clinical rheumatology 28.1 (2014): 31-60.

[94] Frost, Harold M. "Wolff's Law and bone's structural adaptations to mechanical usage: an overview for clinicians." The Angle Orthodontist 64.3 (1994): 175-188.

[95] Federico, S., and W. Herzog. "Micro-structural models of articular cartilage." Journal of Biomechanics 39 (2006): S407.

[96] Suri, Sunita, and David A. Walsh. "Osteochondral alterations in osteoarthritis." Bone 51.2 (2012): 204-211.

[97] Brown, M. A., and R. C. Semelka. "MRI: basic principles and applications" John Wiley\&Sons. (2003): 197-212.

[98] Fleisch, Daniel. "Faraday's Law". A student's guide to Maxwell's Equations. Cambridge University Press, Cambridge, 2008.

[99] Weinmann, Hanns-Joachim et al. "Characteristics of Gadolinium-DTPA Complex: A potential NMR Contrast Agent". American Journal of Roentgenology, vol 142, no. 3, March 1984, pp 610-624. 
[100] "Gadobutrol." American Journal of Health-System Pharmacy, vol. 68, no. 9, 2011, p. 768. Academic OneFile

[101] Crank, J., and P. Nicolson. "A Practical Method for Numerical Evaluation of Solutions of Partial Differential Equations of the Heat-Conduction Type." Advances in Computational Mathematics, vol. 6, no. 3-4, 1996, pp. 207-226.

[102] Maroudas, A. "Distribution and diffusion of solutes in articular cartilage." Biophysical journal 10.5 (1970): 365-379.

[103] Lakowicz, Joseph R., and Barry R. Masters. "Principles of fluorescence spectroscopy." Journal of Biomedical Optics 13.2 (2008): 029901.

[104] Valeur, Bernard, and Mário Nuno Berberan-Santos. Molecular fluorescence: principles and applications. John Wiley \& Sons, 2012.

[105] Lakowicz, Joseph R. "Introduction to fluorescence." Principles of fluorescence spectroscopy. Springer US, 1999. 1-23.

[106] Prentice, A. I. D. "Autofluorescence of bone tissues." Journal of clinical pathology 20.5 (1967): 717-719.

[107] Armstrong, W. G., and Helena Joan Horsley. "Isolation of Fluorescent Components from Ox-bone Human Dentine and Gelatine." Nature 211 (1966): 981.

[108] Manning, Hugh B., et al. "Detection of cartilage matrix degradation by autofluorescence lifetime." Matrix Biology 32.1 (2013): 32-38.

[109] Mondal, Partha Pratim, and Alberto Diaspro. Fundamentals of fluorescence microscopy: exploring life with light. Springer Science \& Business Media, 2013.

[110] Murphy, Douglas B. Fundamentals of light microscopy and electronic imaging. John Wiley \& Sons, 2002.

[111] Antonini, E., et al. "Studies on dextran and dextran derivatives. I. Properties of native dextran in different solvents." Biopolymers 2.1 (1964): 27-34.

[112] Titus, Julie A., et al. "Texas red, a hydrophilic, red-emitting flourophore for use with flourescein in dual parameter flow microfluorometric and fluorescence microscopic studies." Journal of immunological methods 50.2 (1982): 193-204.

[113] Arbabi, Vahid, et al. "Multiphasic Modeling of Charged Solute Transport Across Articular Cartilage: Application of Multi-Zone Finite-Bath Model." Journal of Biomechanics, vol. 49, no. 9, 2016, pp. 1510-1517. 
[114] Salo, EN, et al. "Diffusion of Gd-DTPA(2-) into Articular Cartilage." Osteoarthritis and Cartilage, vol. 20, no. 2, 2012, pp. 117-126

[115] Sterner, B., et al. "The effect of polymer size and charge of molecules on permeation through synovial membrane and accumulation in hyaline articular cartilage." European Journal of Pharmaceutics and Biopharmaceutics 101 (2016): 126-136.

[116] Garcia, A. Minerva, et al. "Transport and binding of insulin-like growth factor I through articular cartilage." Archives of biochemistry and biophysics 415.1 (2003): 69-79.

[117] Garcia, A. Minerva, et al. "Contributions of fluid convection and electrical migration to transport in cartilage: relevance to loading." Archives of Biochemistry and Biophysics 333.2 (1996): 317-325.

[118] Walter, Benjamin A. et al. "TNF $\alpha$ Transport Induced by Dynamic Loading Alters Biomechanics of Intact Intervertebral Discs." Ed. Christoph Englert. PLoS ONE 10.3 (2015): e0118358.

[119] Bonassar, Lawrence J., et al. "The effect of dynamic compression on the response of articular cartilage to insulin-like growth factor-I." Journal of Orthopaedic Research 19.1 (2001): 11-17. 


\section{Appendices}

Appendix A - Diffusion chamber engineering drawing

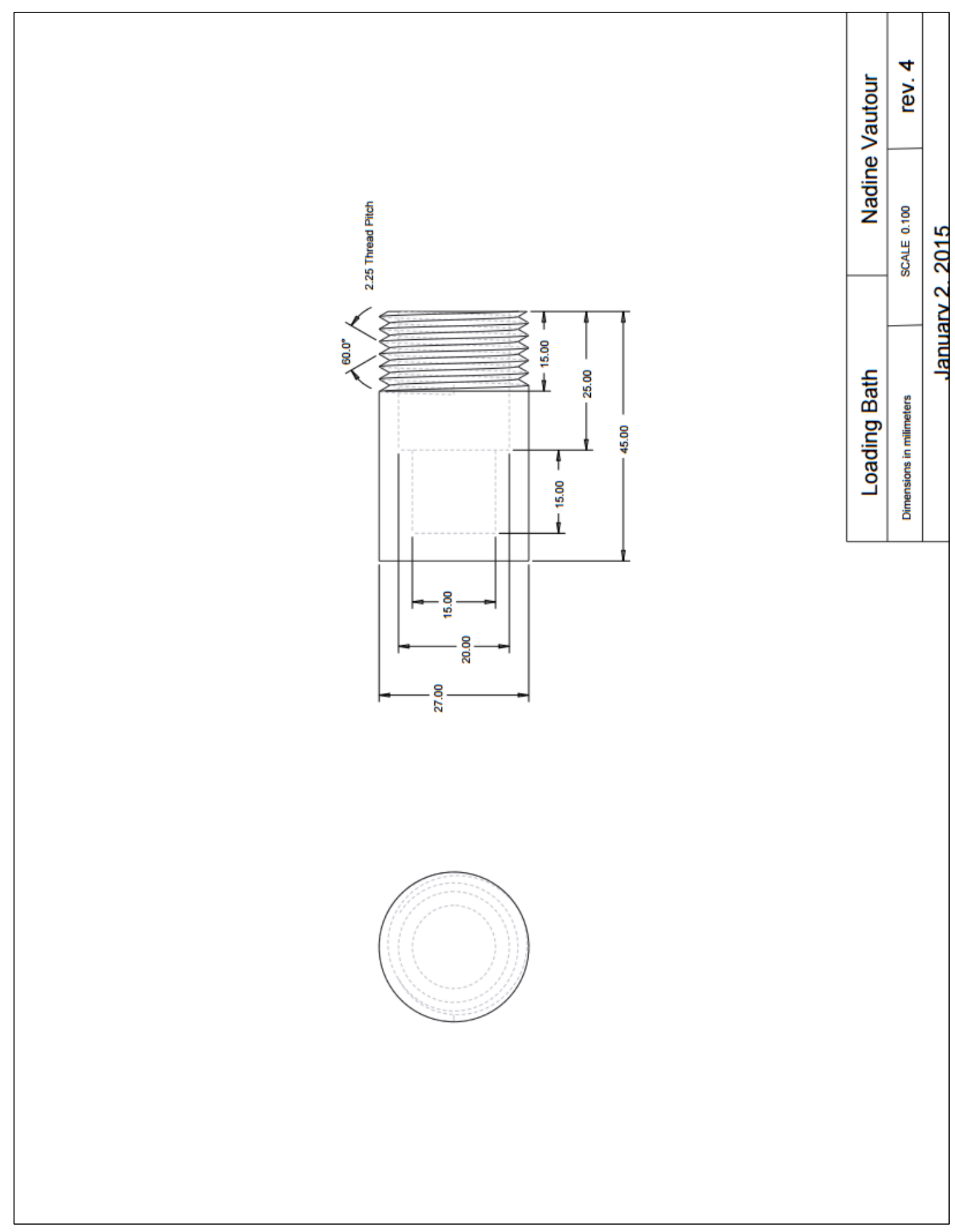




\section{Appendix B - Experimental Gd concentration at select cartilage depths and times}

\section{for all samples}

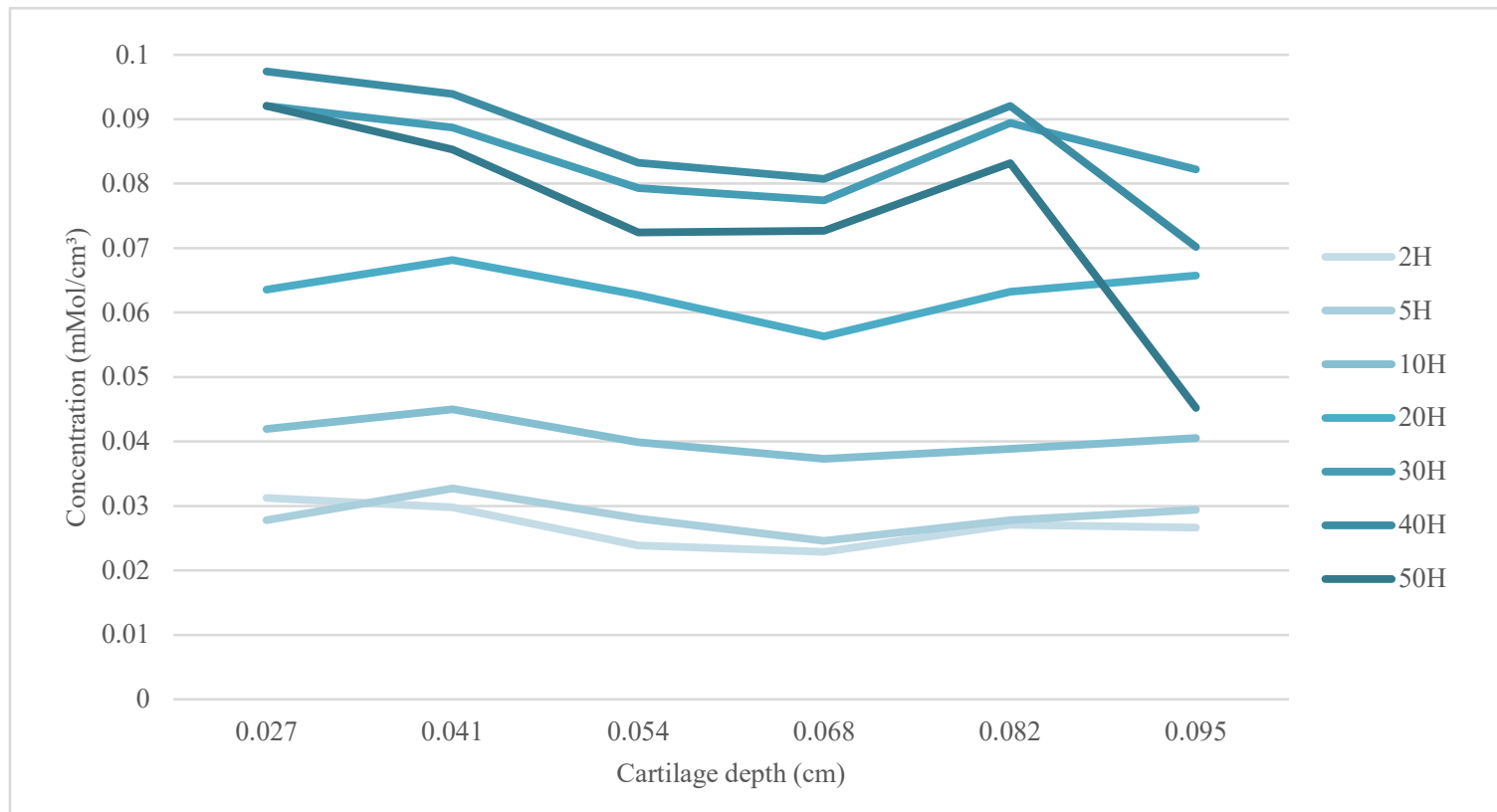

Illustration 25 Gd concentration at various times, throughout the cartilage depth for all sample 1

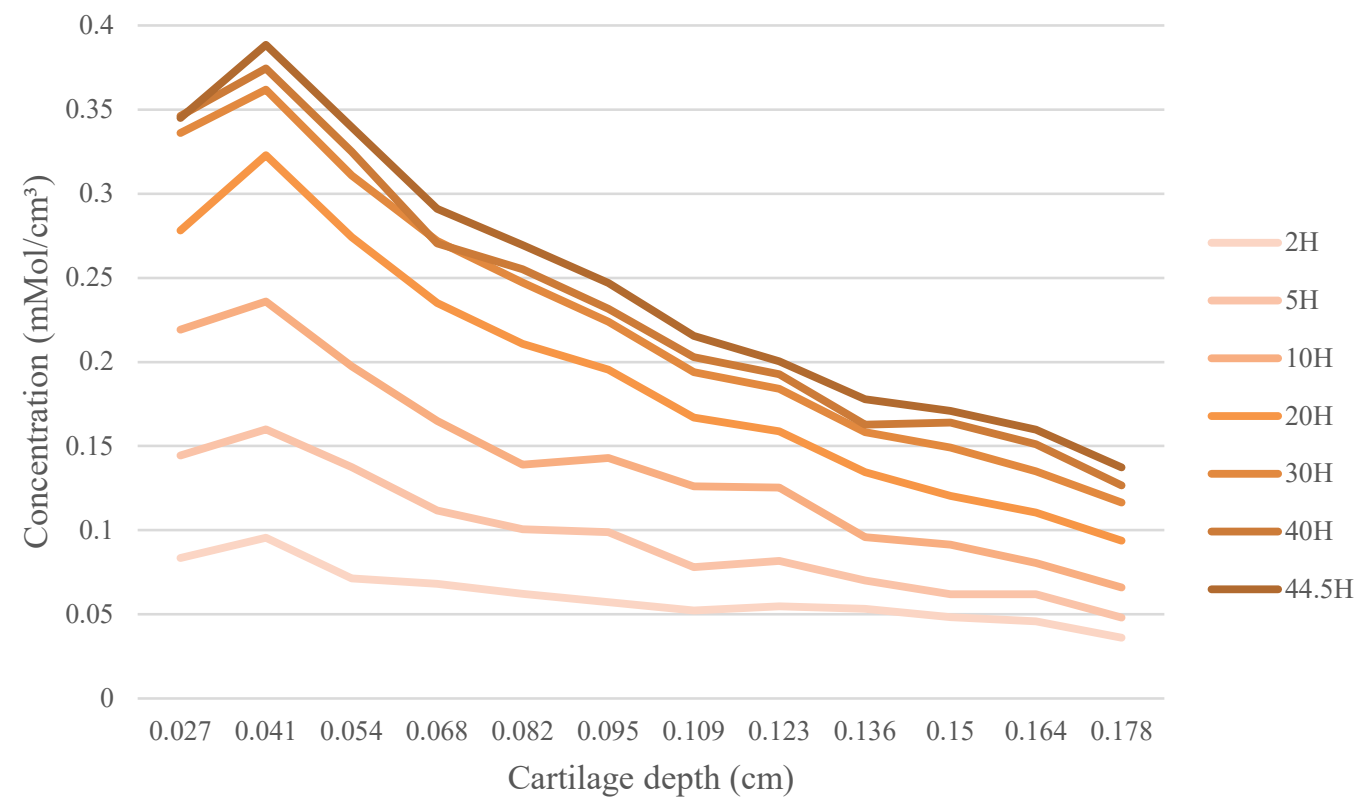

Illustration 26 Gd concentration at various times, throughout the cartilage depth for all sample 2 


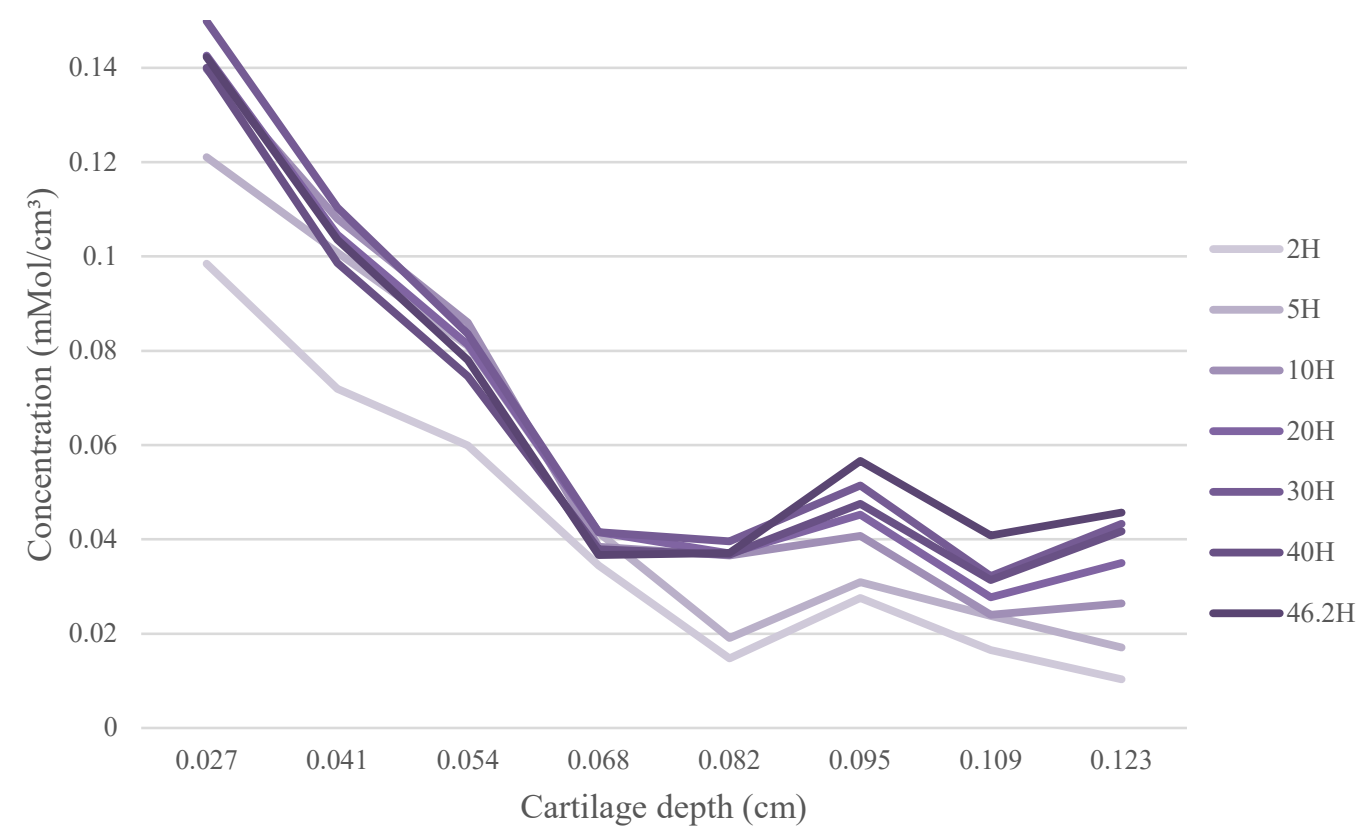

Illustration 28 Gd concentration at various times, throughout the cartilage depth for all sample 3

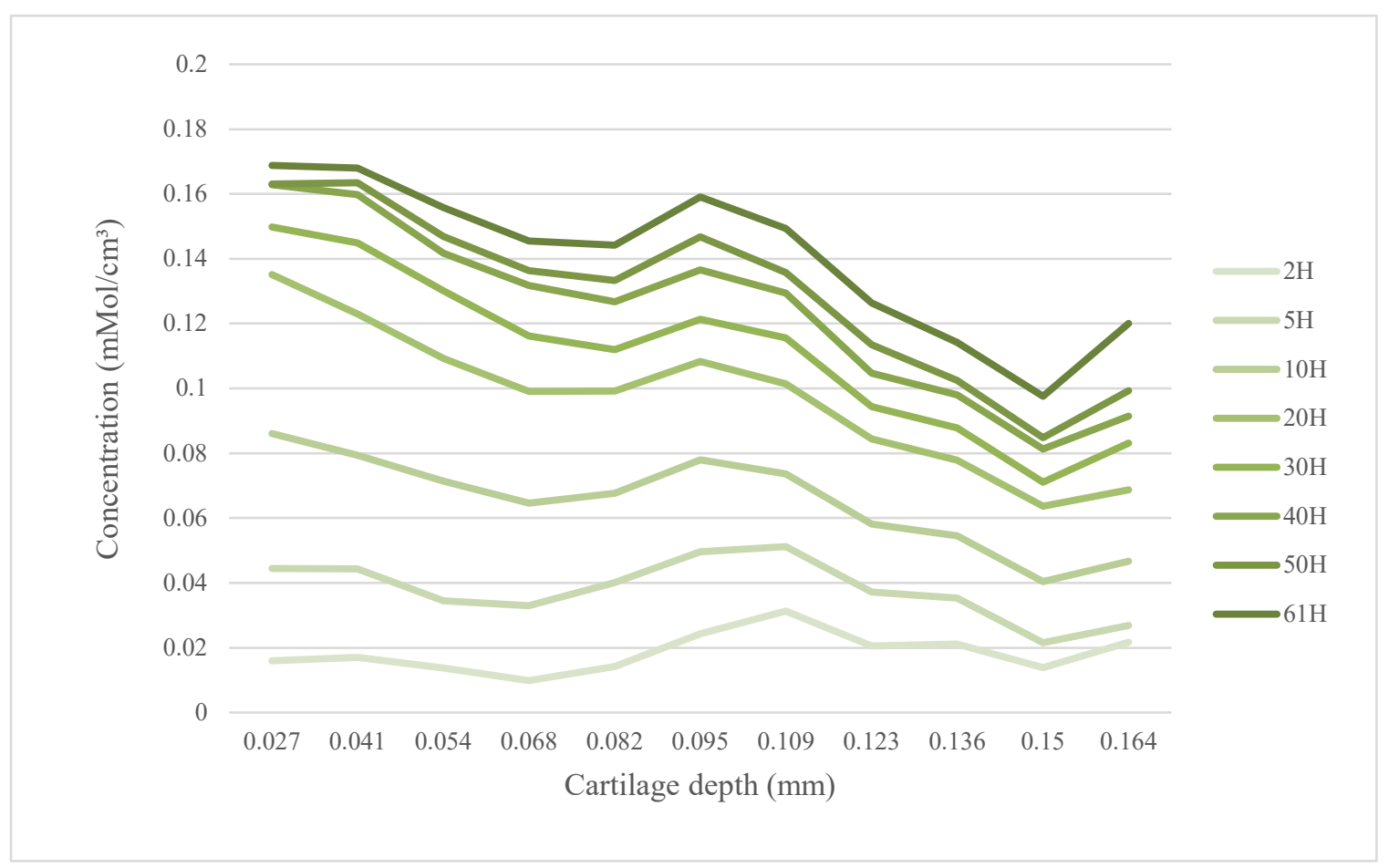

Illustration 27 Gd concentration at various times, throughout the cartilage depth for all sample 4 


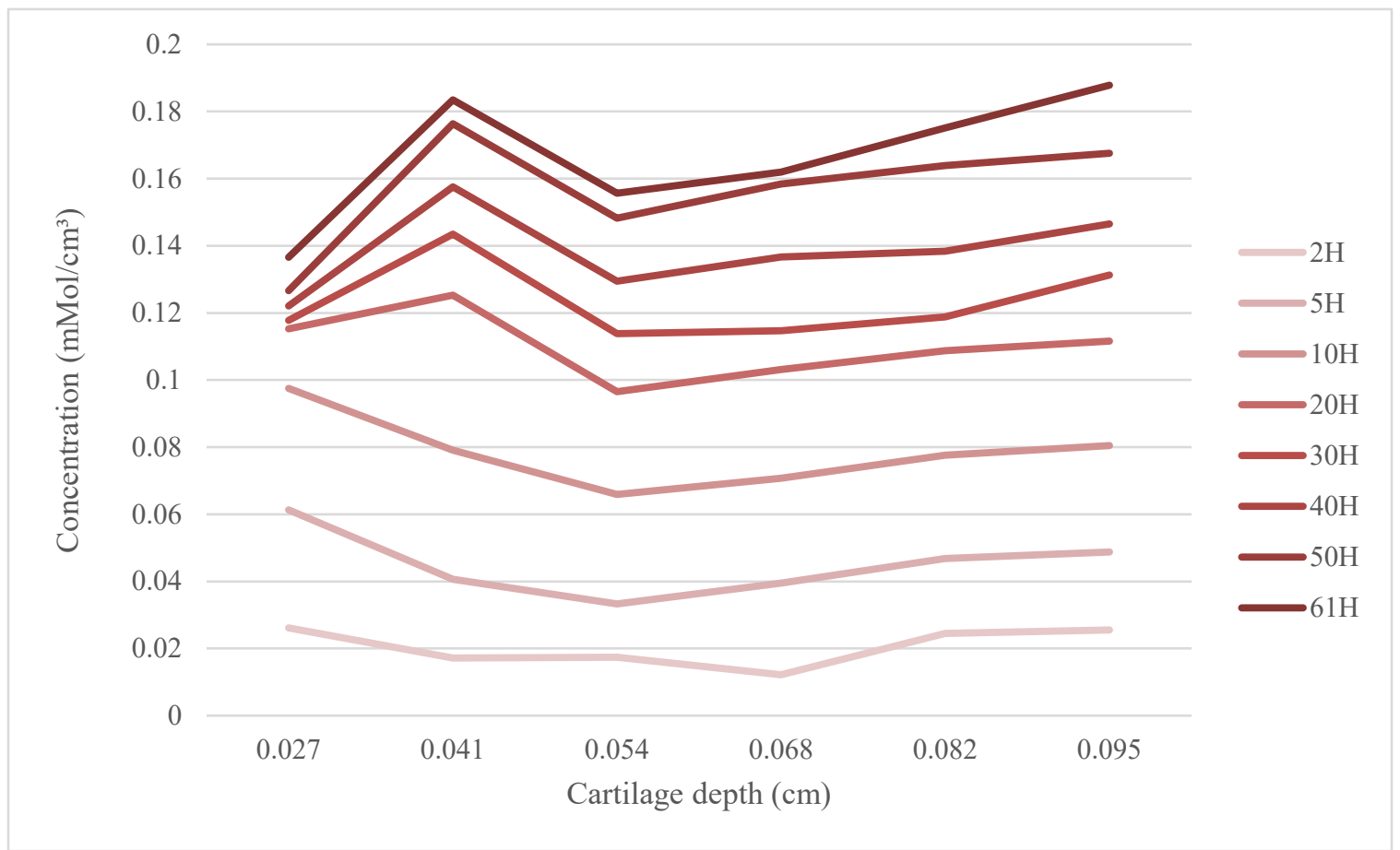

Illustration 30 Gd concentration at various times, throughout the cartilage depth for all sample 5

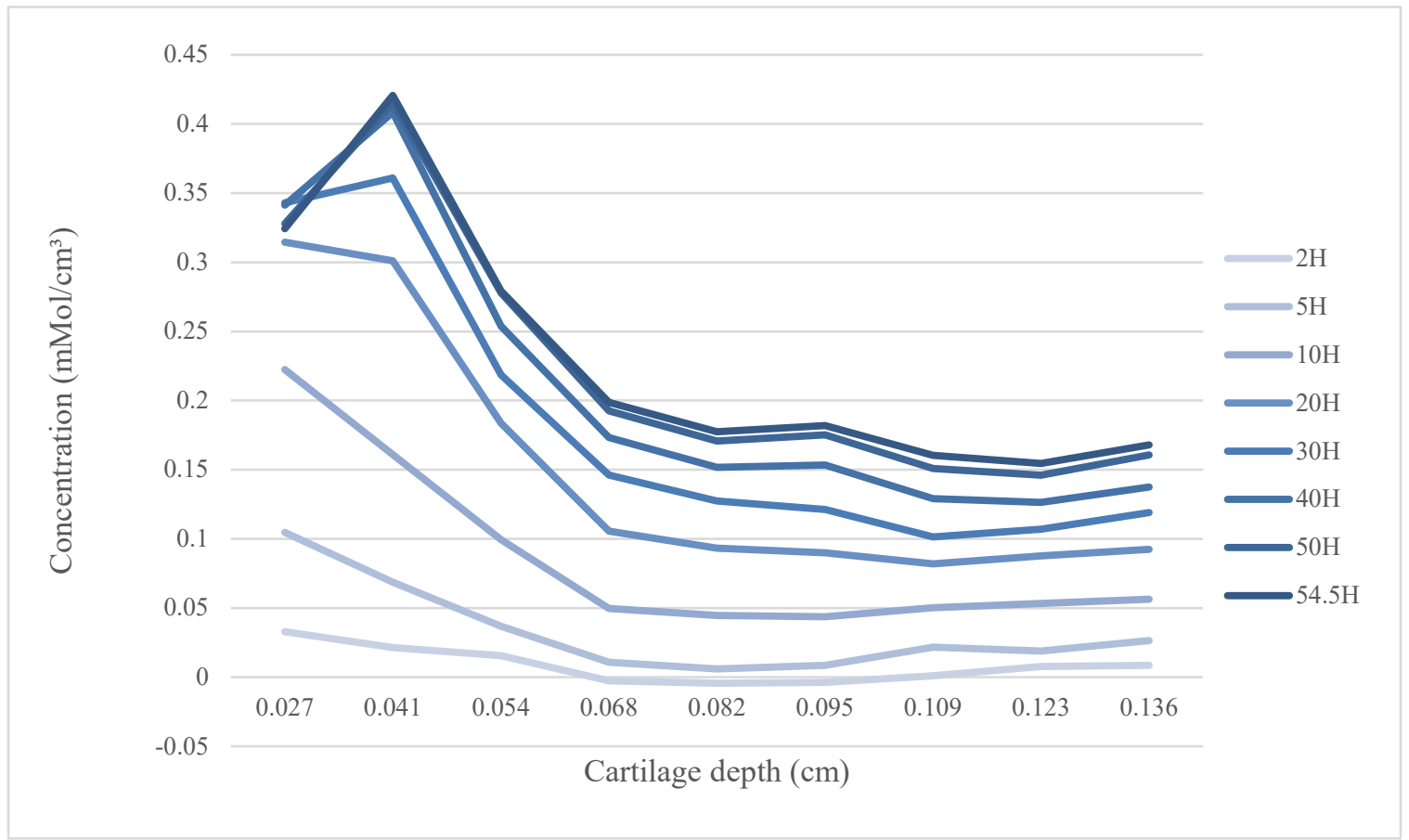

Illustration 29 Gd concentration at various times, throughout the cartilage depth for all sample 6 


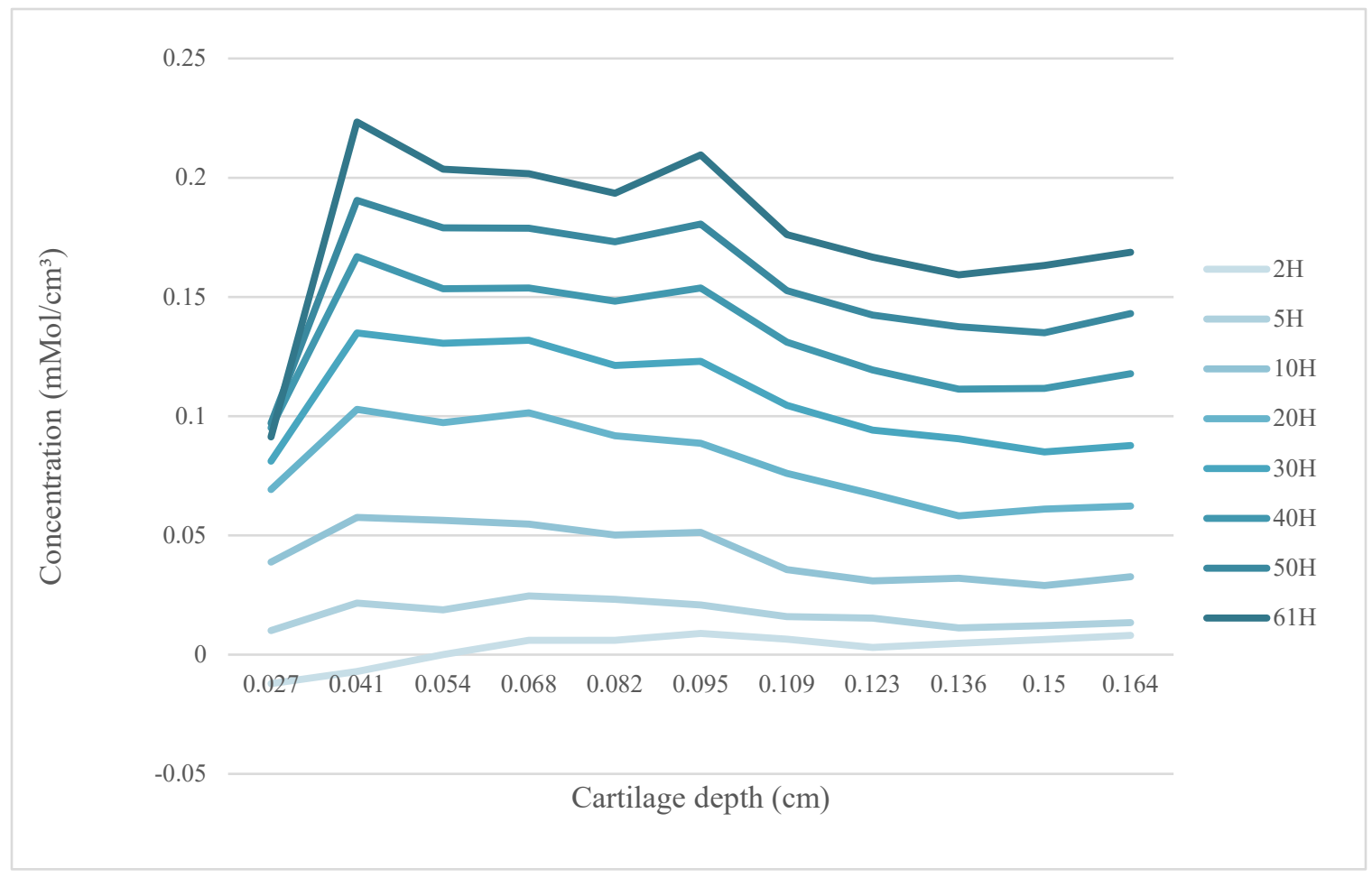

Illustration 31 Gd concentration at various times, throughout the cartilage depth for all sample 7 


\section{Appendix C - Simulated Gd concentration fit to experimental results at select cartilage depths and times, for all samples}

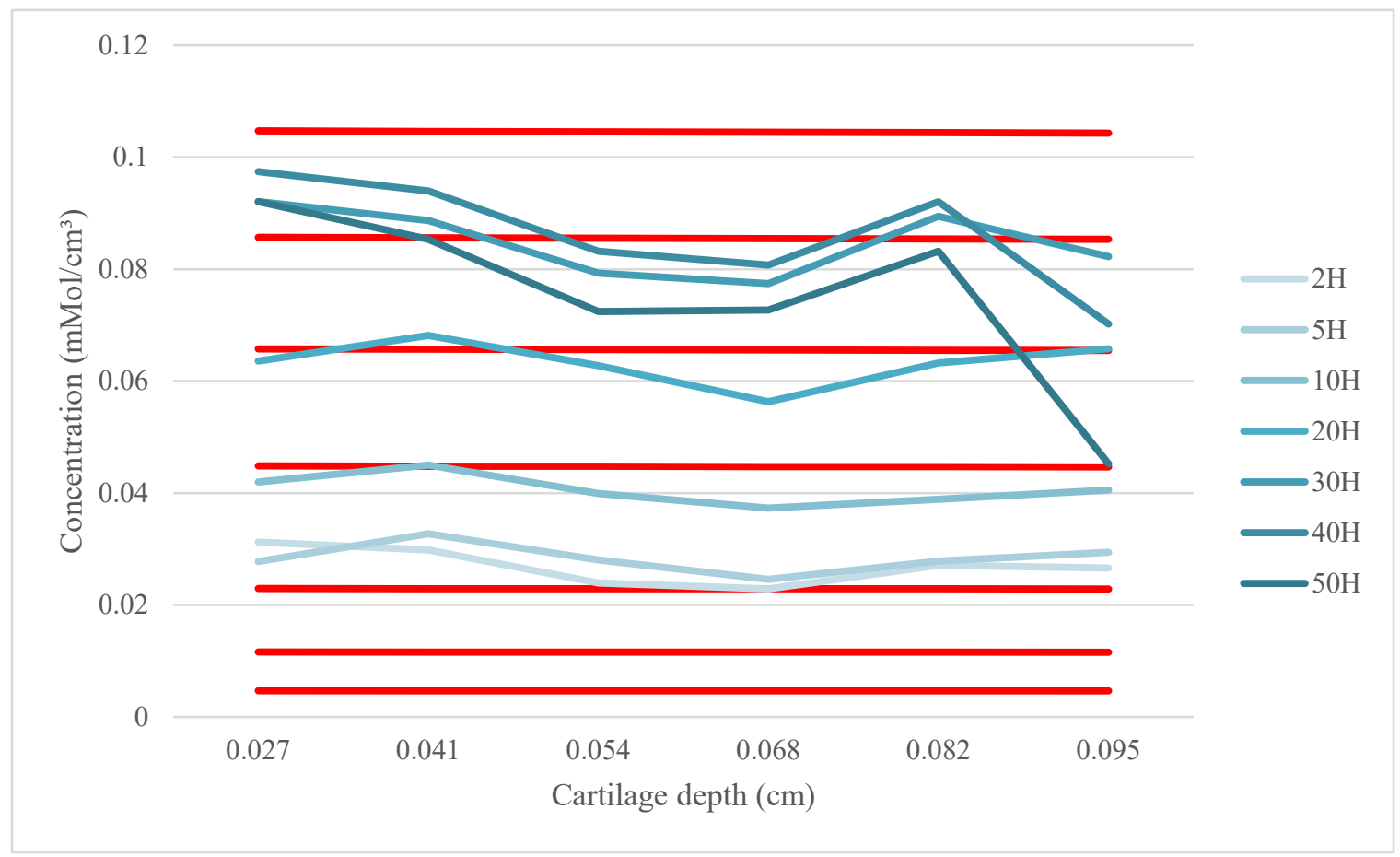

Illustration 32 Sample 1: simulated fit to experimental concentrations

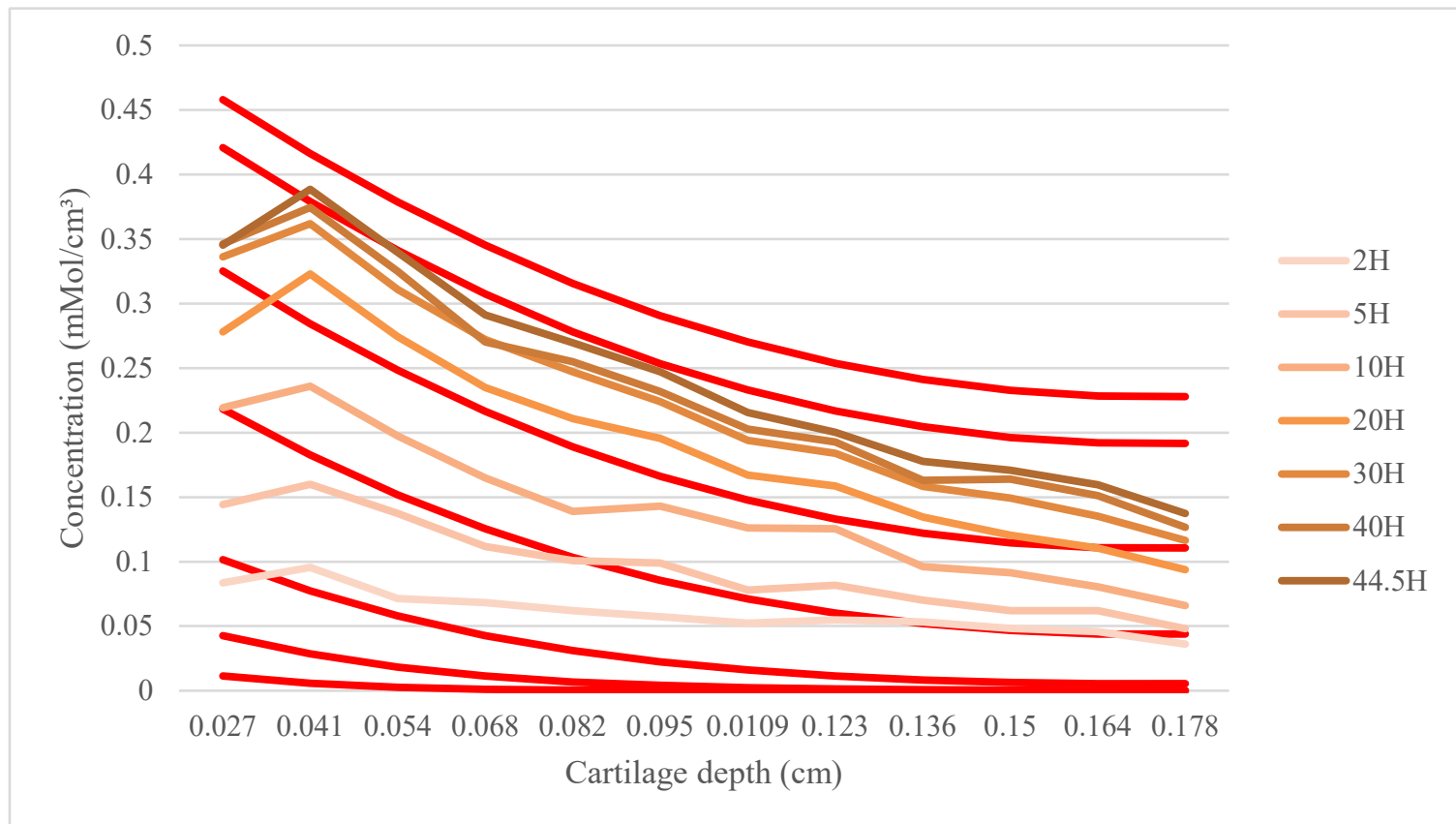

Illustration 33 Sample 2: simulated fit to experimental concentrations 


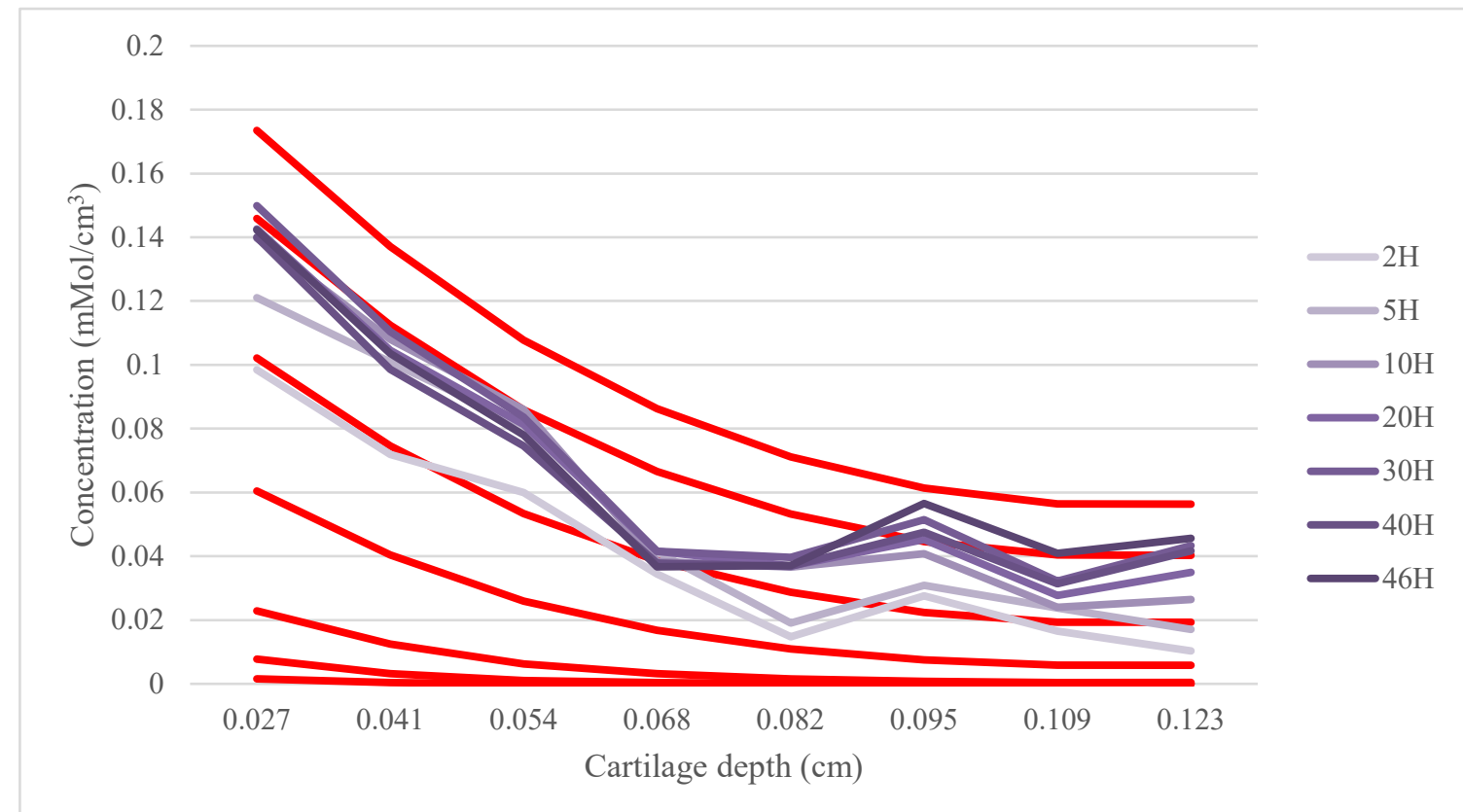

Illustration 34 Sample 3: simulated fit to experimental concentrations

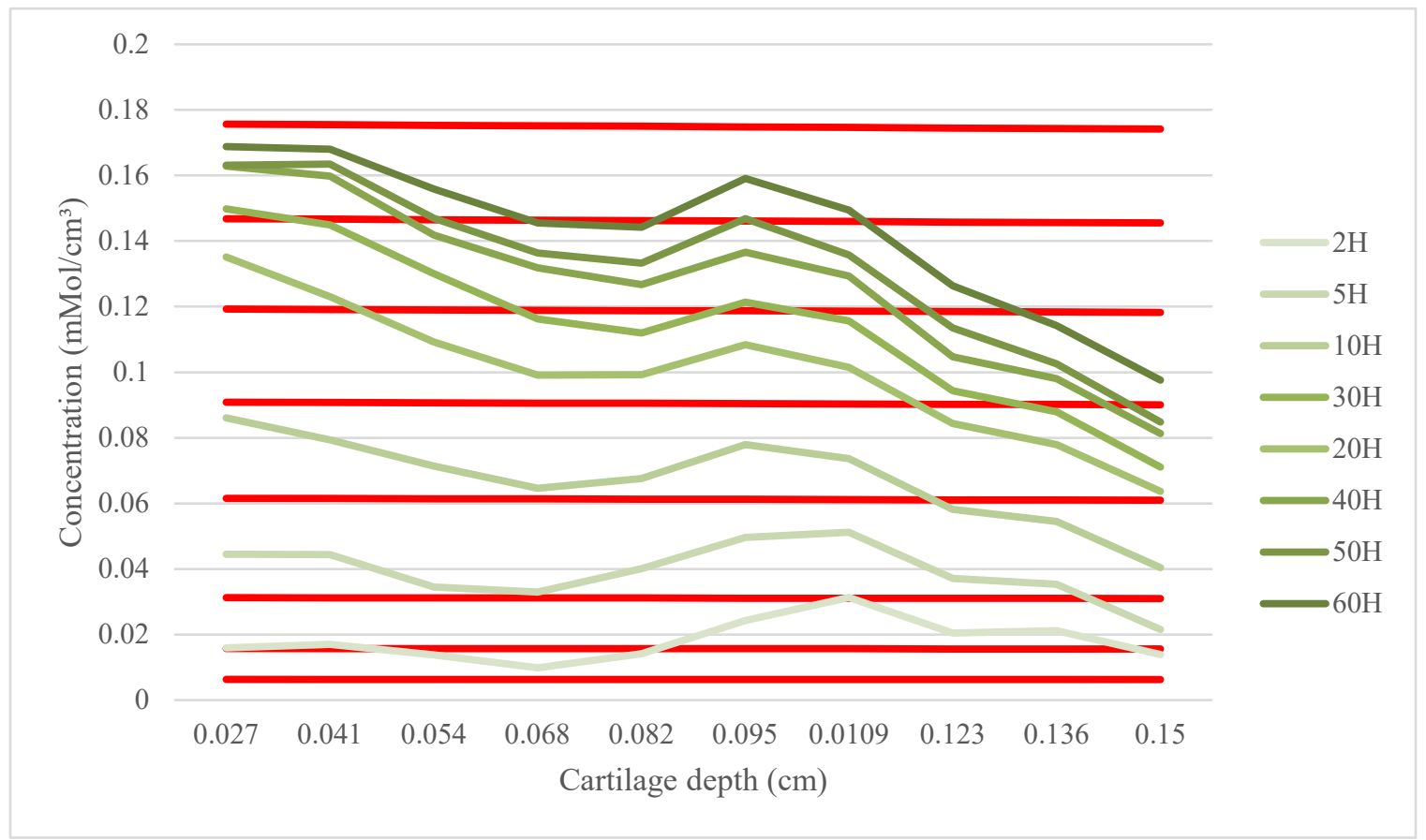

Illustration 35 Sample 4: simulated fit to experimental concentrations 

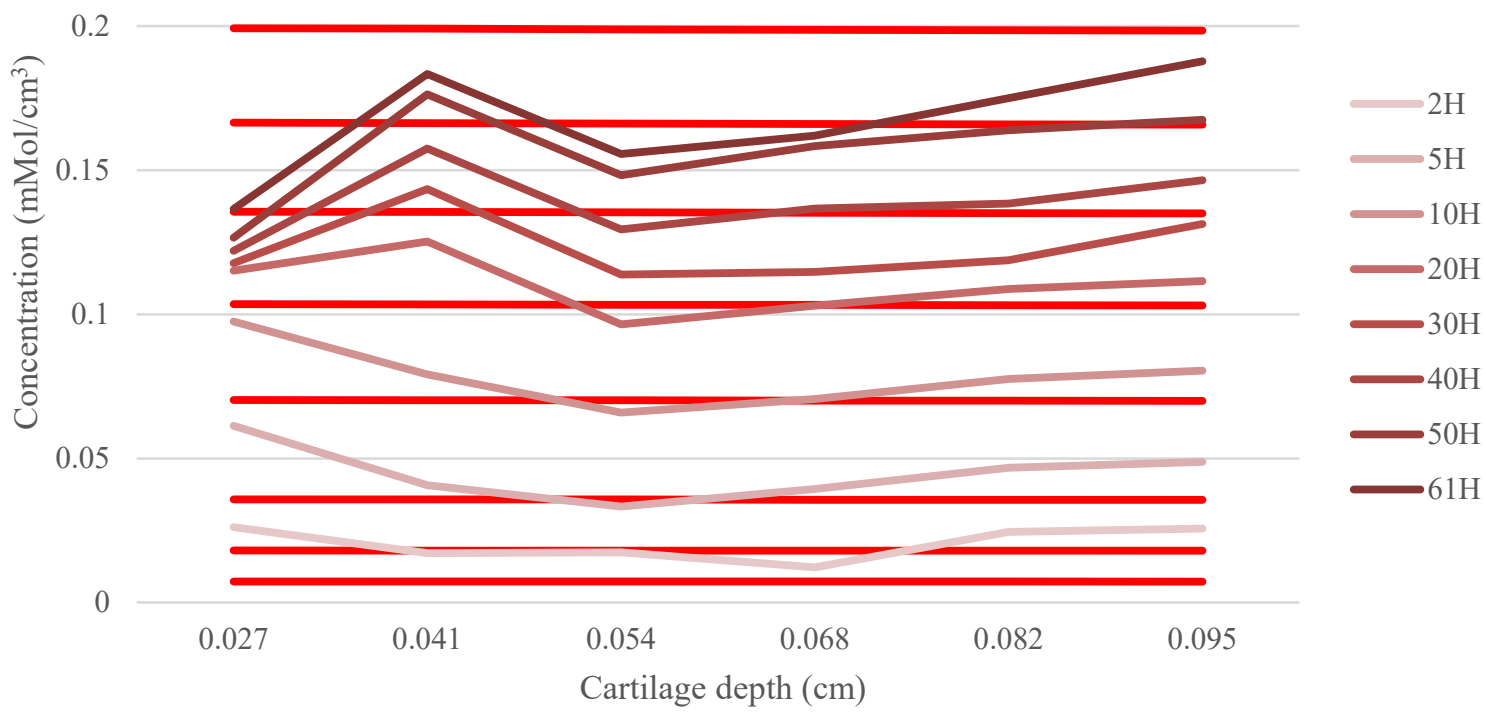

Illustration 36 Sample 5: simulated fit to experimental concentrations

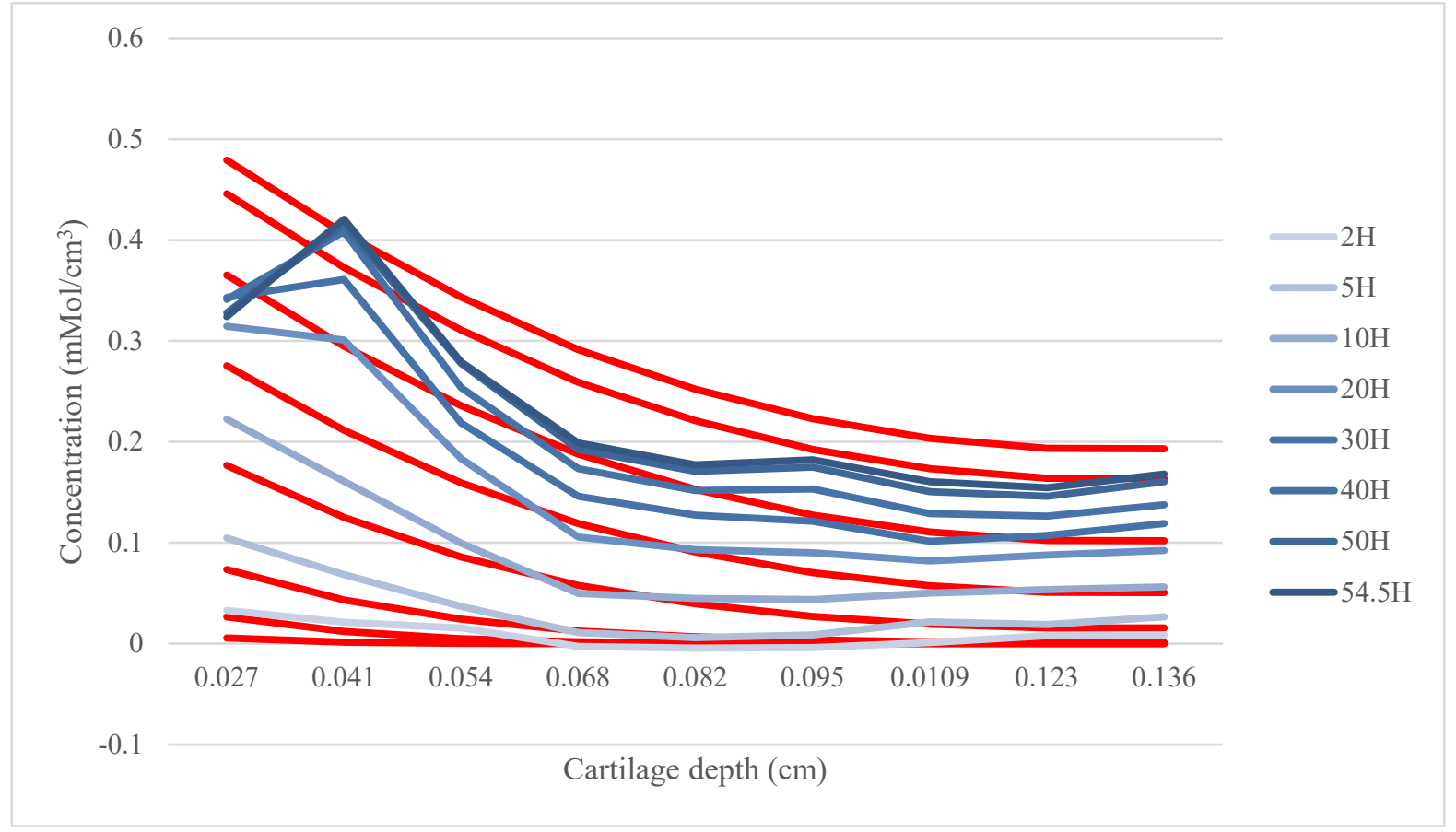

Illustration 37 Sample 6: simulated fit to experimental concentrations 


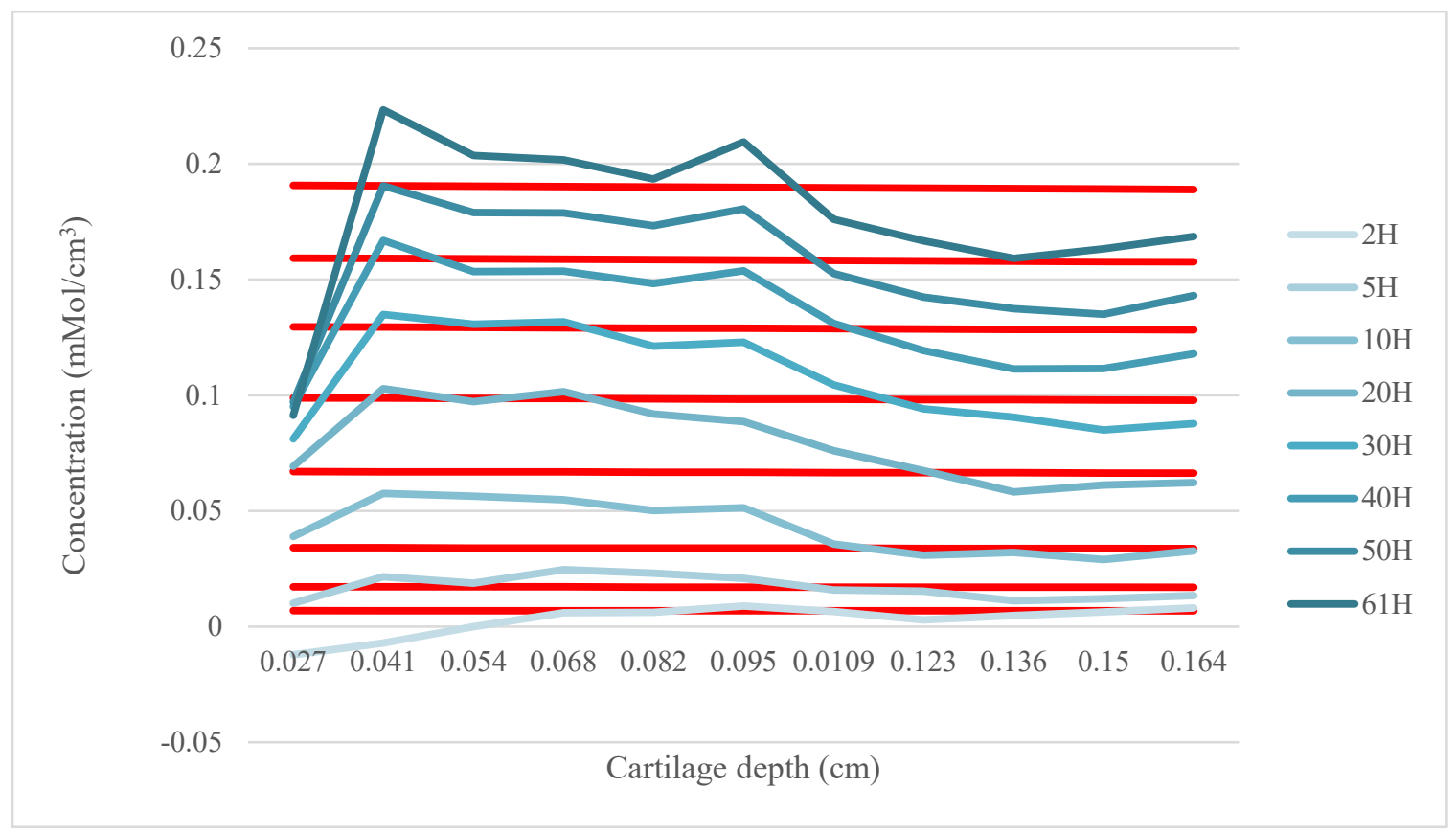

Illustration 38 Sample 7: simulated fit to experimental concentrations 


\section{Appendix D Simulated Gd concentration without the bone barrier fit to}

experimental results at select cartilage depths and times, for all samples

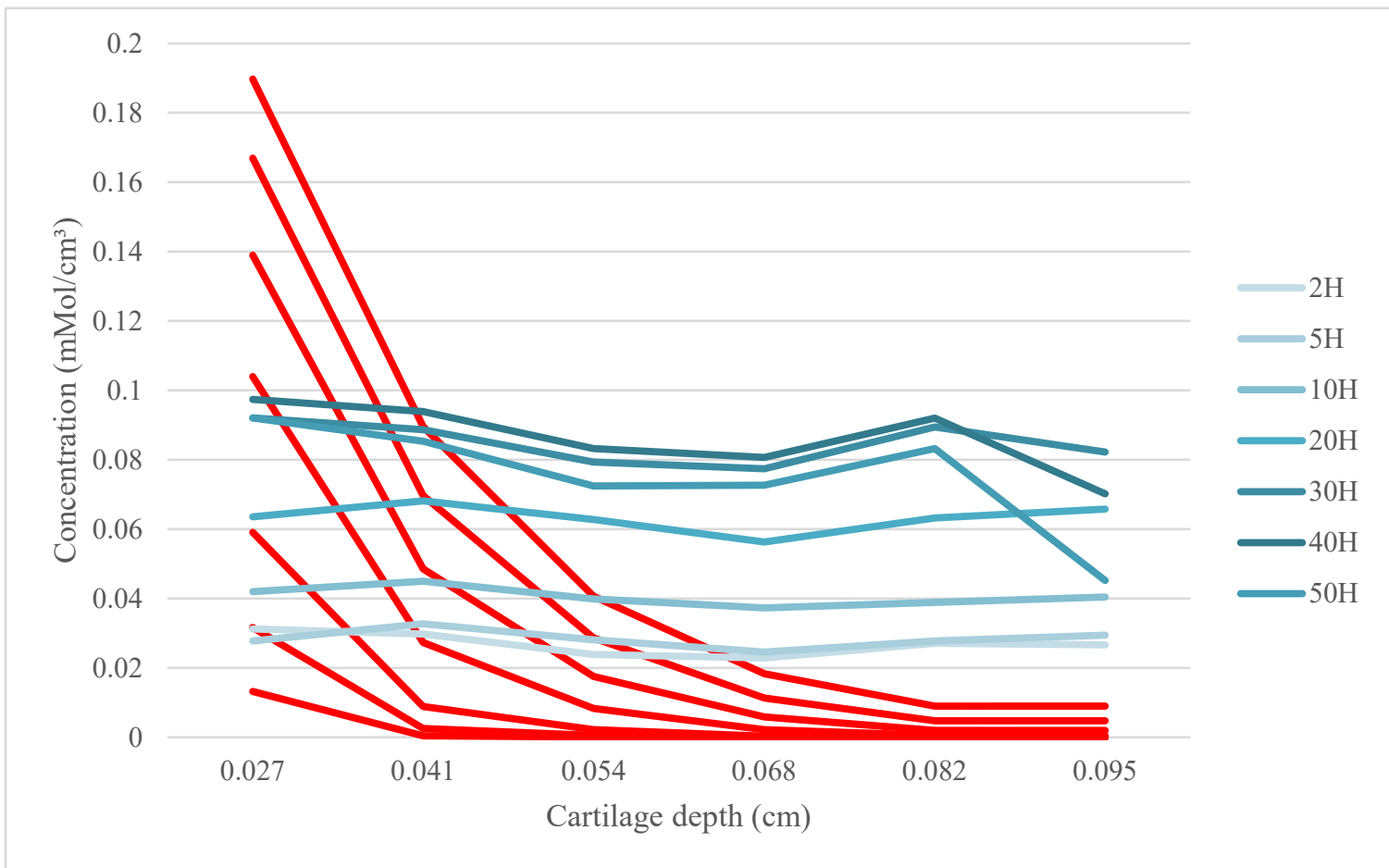

Illustration 39 Sample 1: simulated fit to experimental concentrations, without the bone barrier 


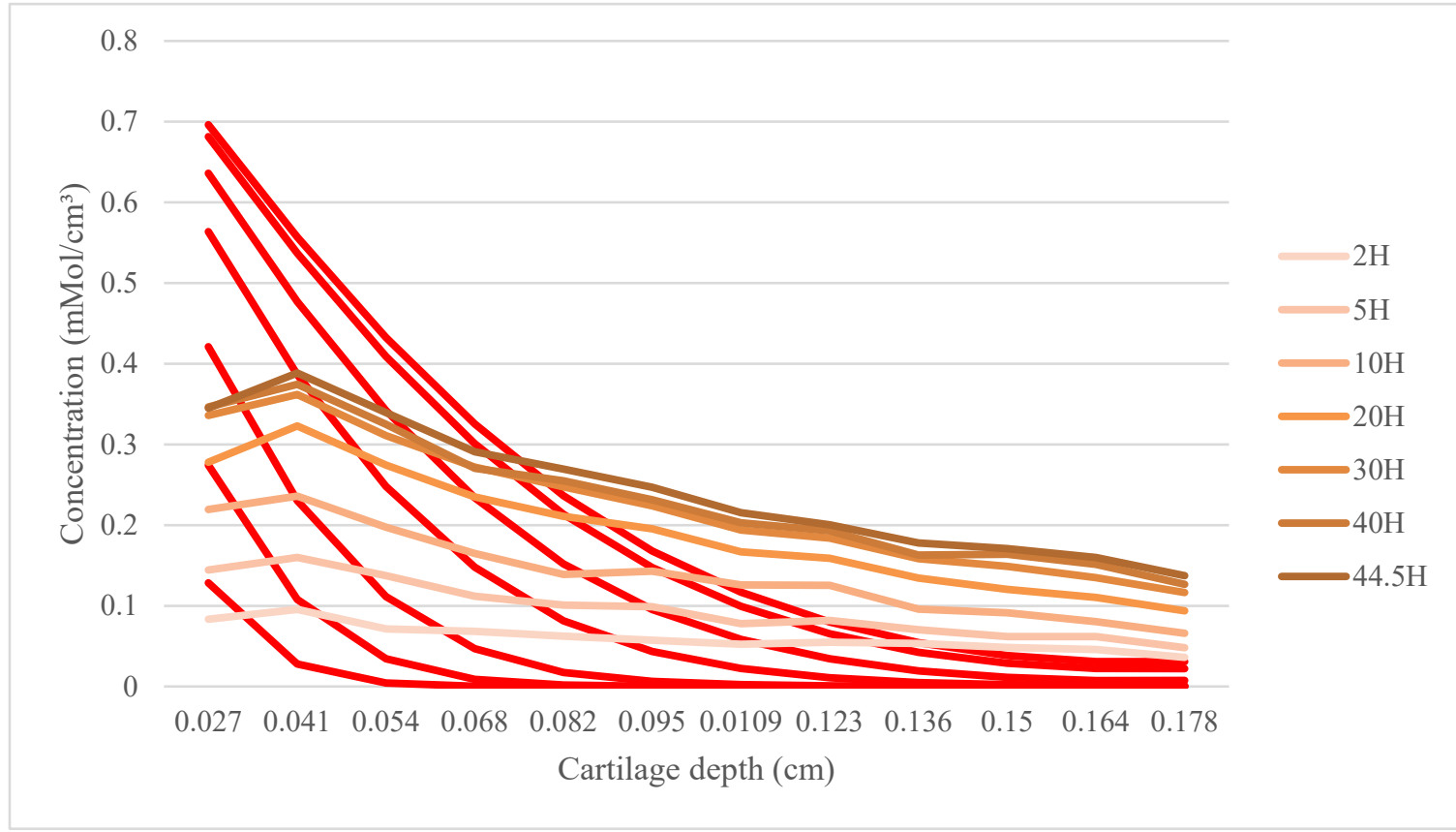

Illustration 40 Sample 2: simulated fit to experimental concentrations, without the bone barrier

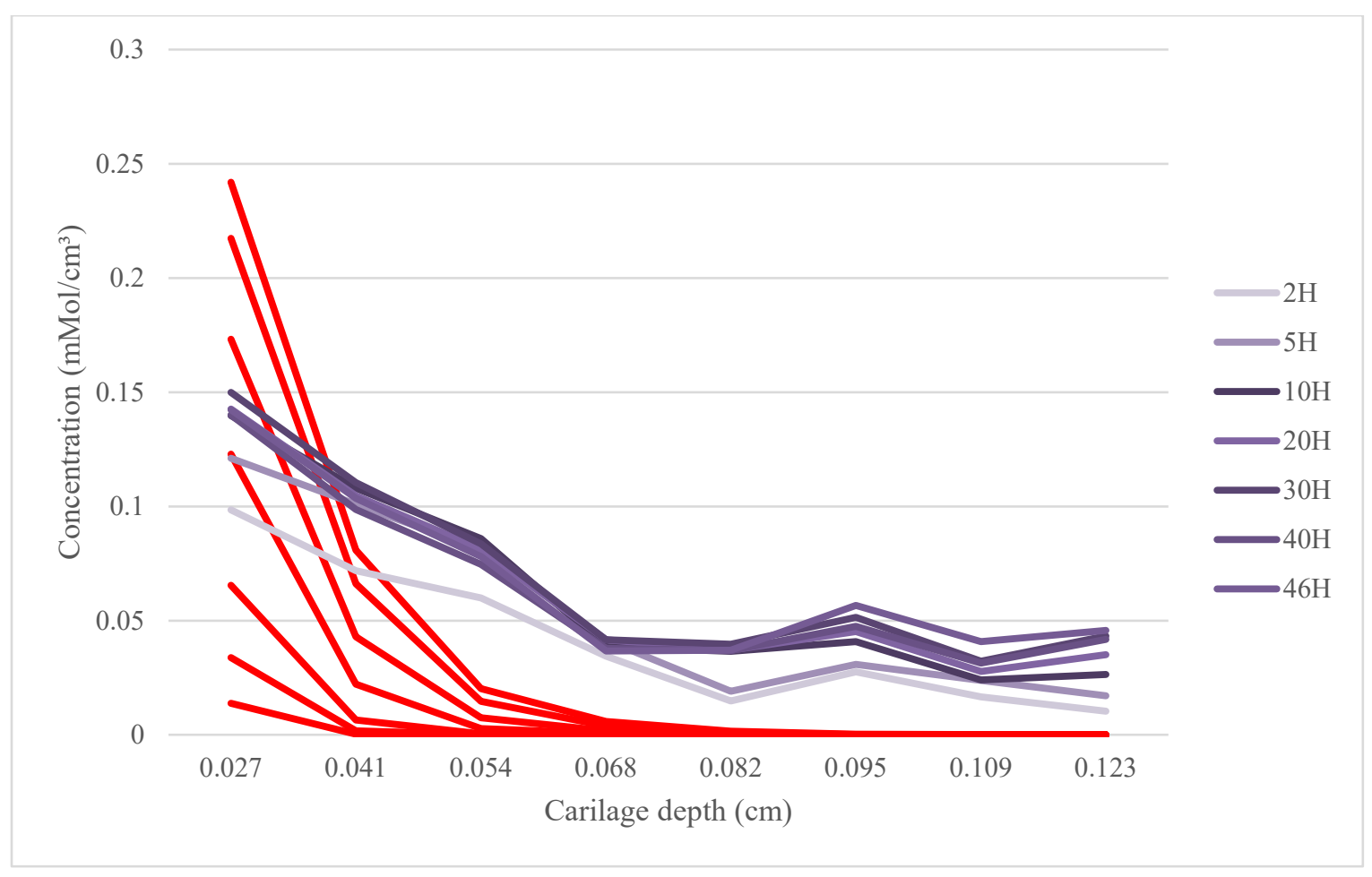

Illustration 41 Sample 3: simulated fit to experimental concentrations, without the bone barrier 


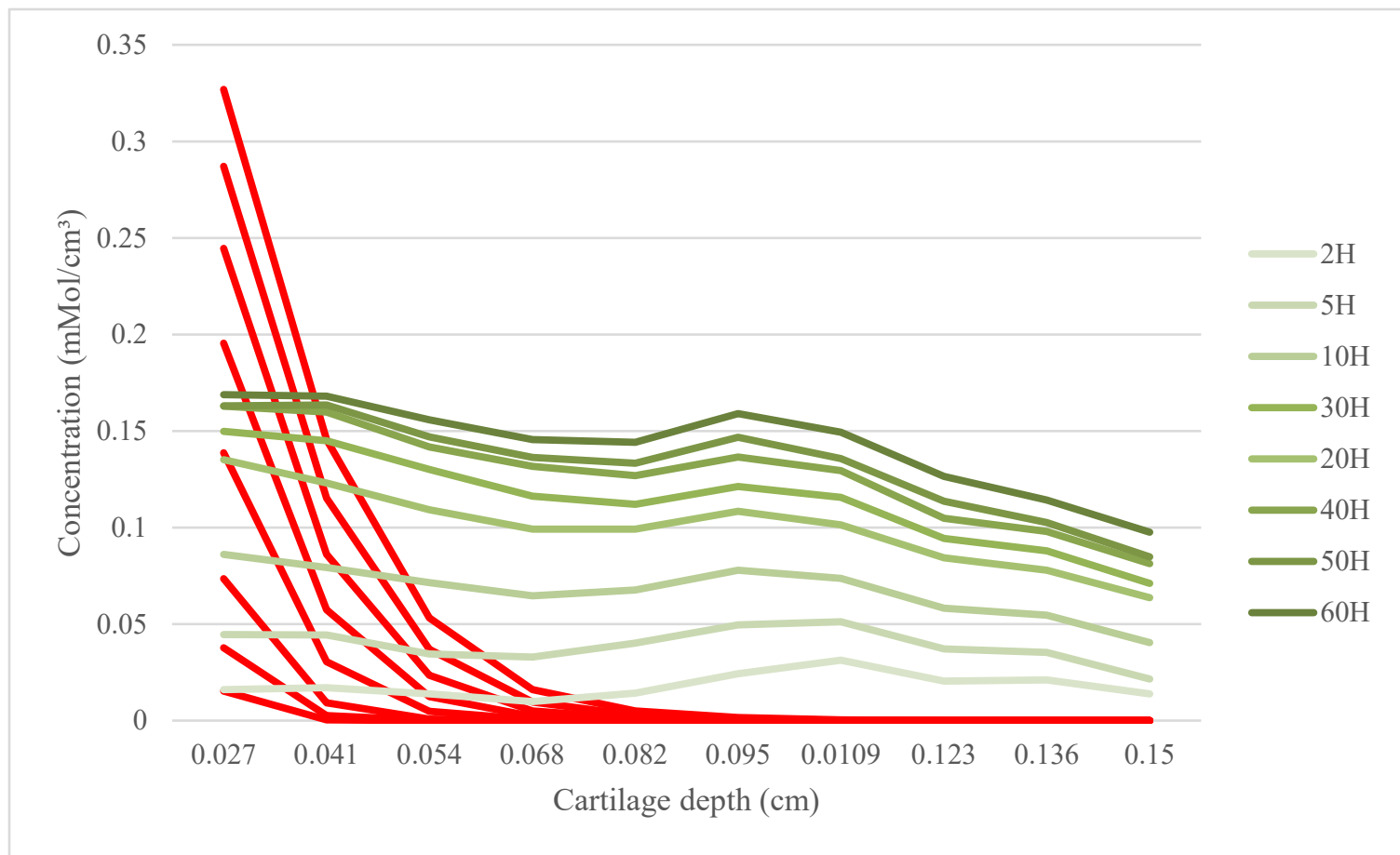

Illustration 42 Sample 4: simulated fit to experimental concentrations, without the bone barrier

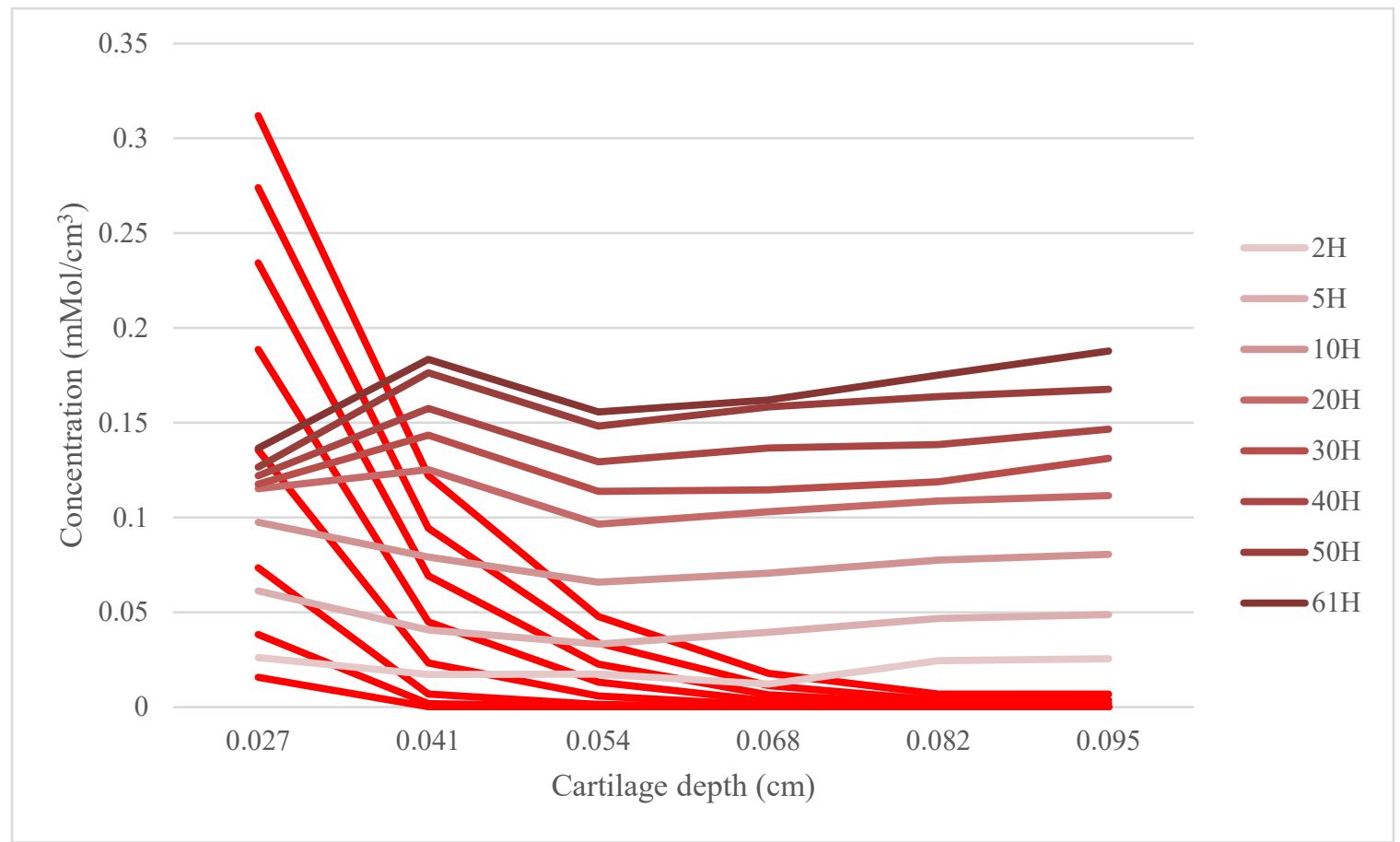

Illustration 43 Sample 5: simulated fit to experimental concentrations, without the bone barrier 


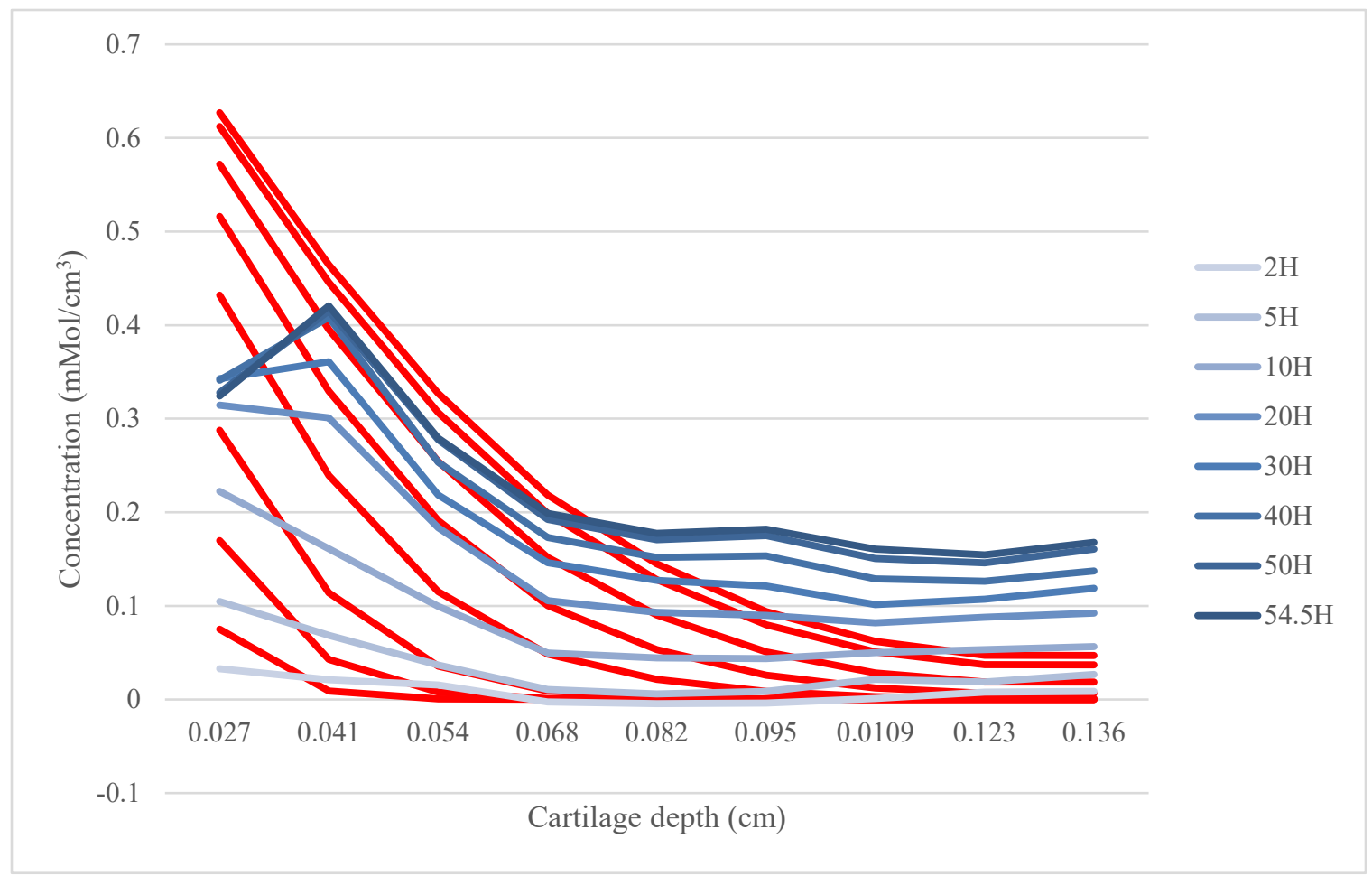

Illustration 44 Sample 6: simulated fit to experimental concentrations, without the bone barrier

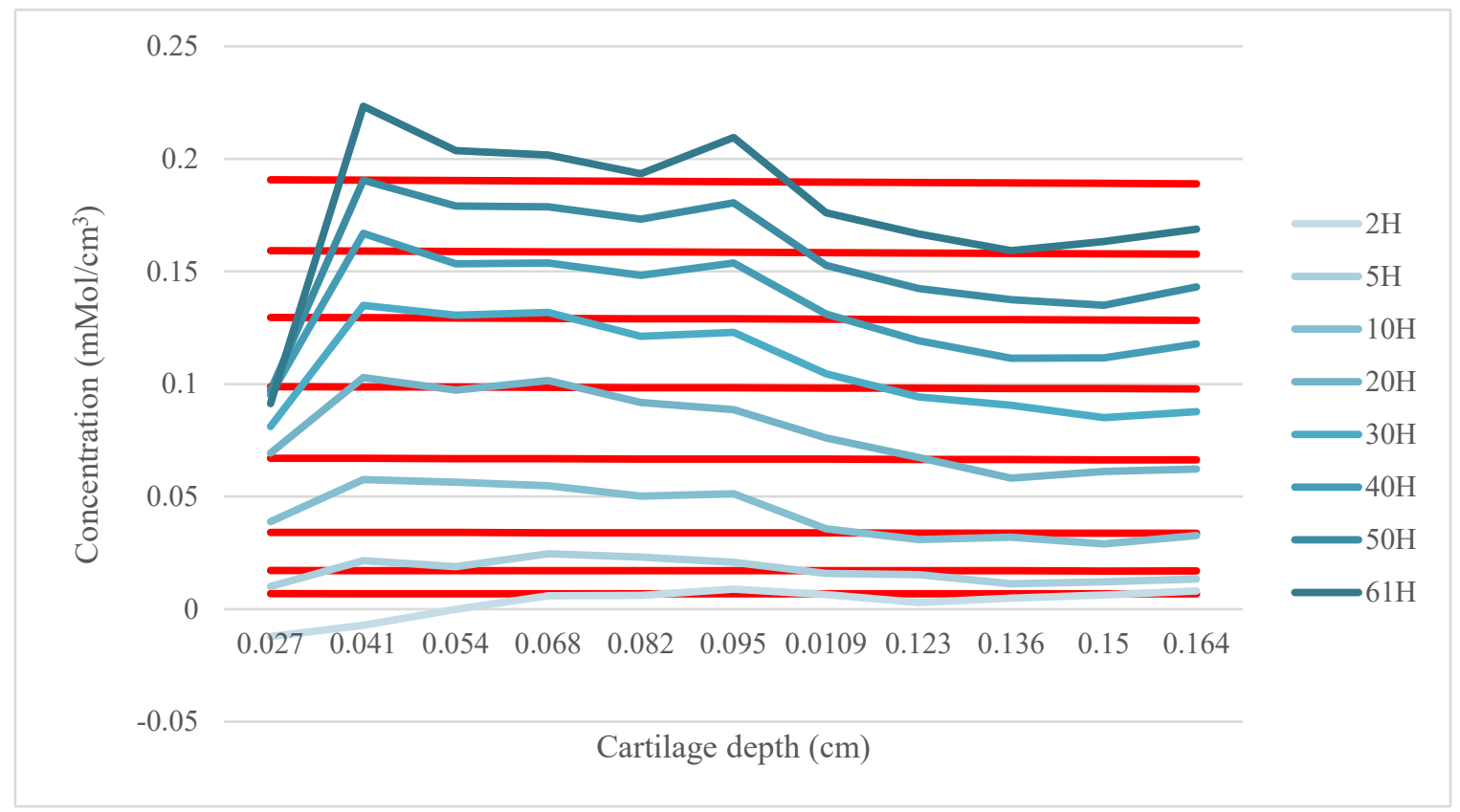

Illustration 45 Sample 7: simulated fit to experimental concentrations, without the bone barrier 